\title{
Regulatory role of plasma membrane microdomains in cellular stress sensing and growth signaling
}

\author{
Ph.D. Thesis
}

\section{Bálint Csoboz}

\author{
Supervisor: Prof. László Vígh \\ Doctoral School of Biology \\ University of Szeged \\ Institute of Biochemistry \\ Biological Research Centre \\ Hungarian Academy of Sciences
}

2018 


\section{Table of Contents}

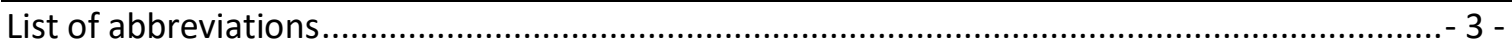

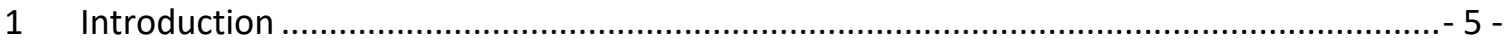

1.1 The composition and organization of the plasma membrane ..................................... 5 -

1.2 Membrane microdomains and the dynamic organization of the membrane .................- 7 -

1.3 Caveolae and their role in signaling compartmentalization ......................................... 9 -

1.4 Membrane microdomains in cellular signaling: the role of membrane fluidity and lateral domain mobility in cellular stress sensing .............................................................. 11 -

1.4.1 Chaperones and the membrane-mediated stress response ...................................... 11 -

1.4.2 The association of small heat shock proteins with membranes.................................. 15 -

1.5 Membrane microdomains at the forefront of disease states: caveolae resident proteins as central players in cancer progression................................................................... 18 -

1.5.1 A group of membrane anchored oncogenes: the PRL family of dual specificity

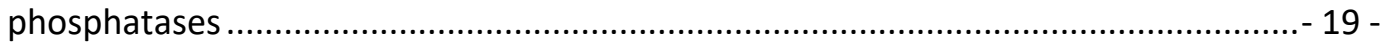

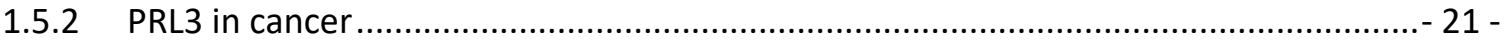

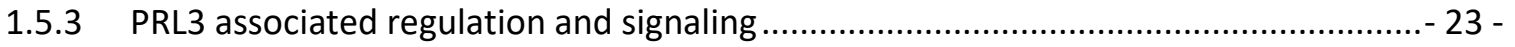

1.5.4 Prenylation and subcellular localization of PRL3 ...................................................... 26 -

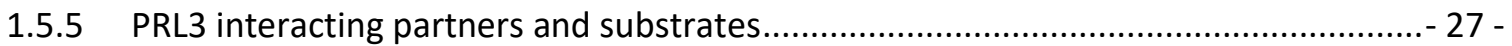

1.5.6 The plausible transmission of PRL3 mediated signaling through integrin receptors ...- 30 -

1.5.7 The role of Rac1 as a possible effector of the PRL3 mediated oncogenesis ................- 30 -

1.5.8 Rac1 control over cell proliferation ....................................................................... 32 -

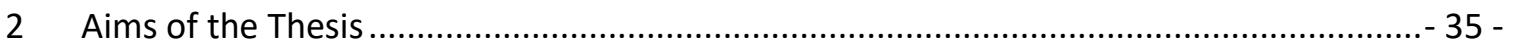

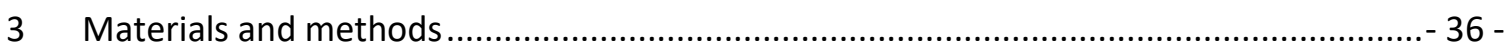

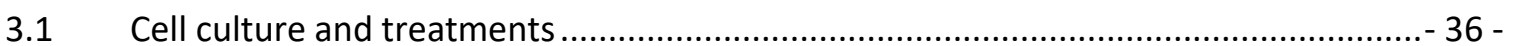

3.2 Transfection, clone selection and stable expression of PRL3 in B16F0 cells ................. 36 -

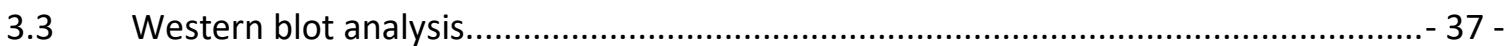

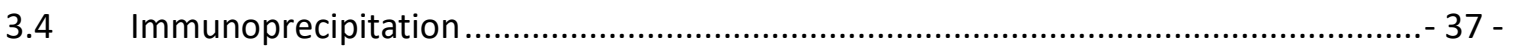

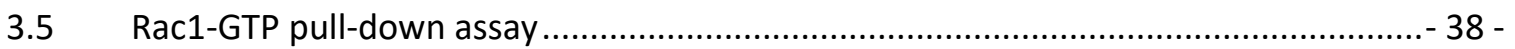

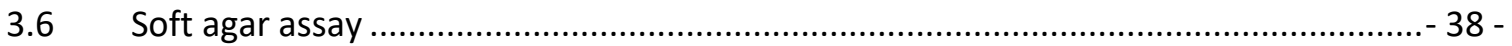

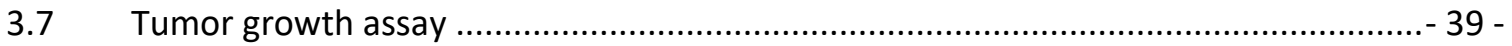

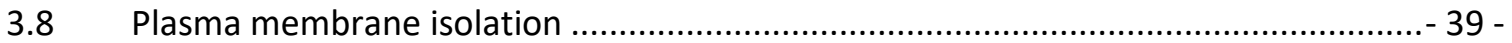

3.9 Detergent-free purification of caveolin enriched membrane fractions .......................- 40 -

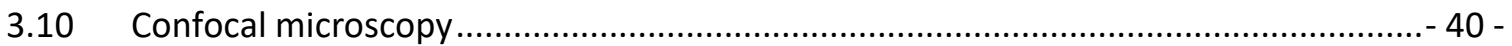

3.11 Imaging of di-4-ANNEPDHQ and calculation of General Polarization (GP) ..................- 41 -

3.12 Preparation of plasma membrane sheets .............................................................. 41 -

3.13 Image based Fluorescent Correlation Spectroscopy (ImFCS) ..................................... 41 - 
3.14 Domain size analysis of sphingomyelin rich microdomains .................................... 43 -

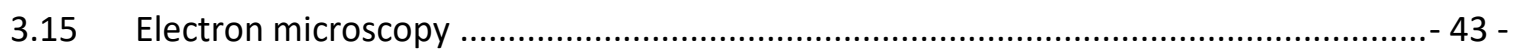

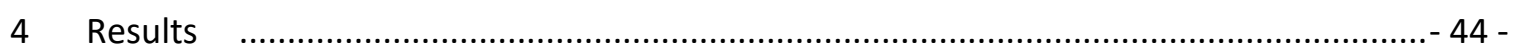

4.1 Membrane microdomains in cellular signaling: the role of membrane fluidity and lateral domain mobility in cellular stress sensing: .......................................................... 44 -

Heat and benzyl alcohol fluidizes the membrane in a similar manner ........................ 44 -

4.2 Membrane fluidization increases the coalescence of sphingomyelin rich membrane domains on the cell surface ...................................................................................... 45 -

4.3 Membrane fluidization mediated membrane domain rearrangement is coupled with

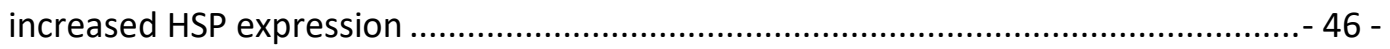

4.4 The membrane fluidization induced HSP expression translates into the acquisition of

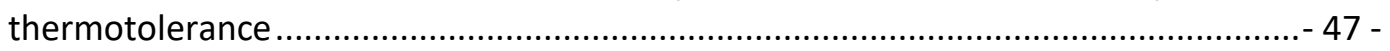

4.5 The artificial expression of HSP25 buffers the fluidizing effect of benzyl alcohol........- 48 -

4.6 The expression of HSP25 alters the membrane domain structure and affects the redistribution of membrane microdomains ......................................................... 50 -

4.7 Membrane proteins as central players in cancer progression: ....................................- 53 -

PRL3 expression is upregulated upon exposure to genotoxic stress............................. 53 -

4.8 B16 cells show enhanced growth properties upon PRL3 expression ........................... 56 -

4.9 PRL3 associates with the plasma membrane and localizes to the caveolae .................-58-

4.10 PRL3 dephosphorylates integrin $\beta 1$ at Thr788/789 .............................................. 62 -

4.11 The presence of PRL3 alters the activation and plasma membrane targeting of Rac1 - 64 -

4.12 Rac1 activation is connected with elevated levels of cyclin D1.................................. 66 -

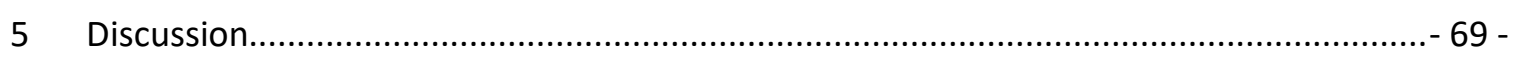

5.1 Structural membrane changes induced chaperon expression contributes to membrane

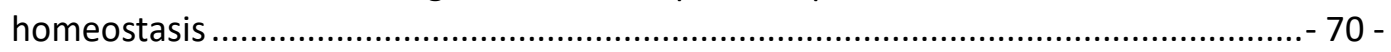

5.2 Chemotherapy induced PRL3 expression promotes cancer growth via plasma membrane remodeling and specific alterations of caveolae-associated signaling......................... 74 -

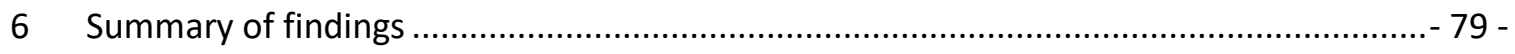

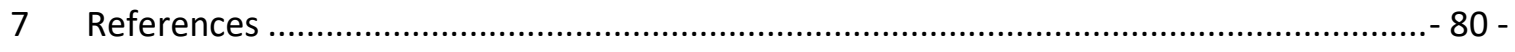

8 Summary- 96 -

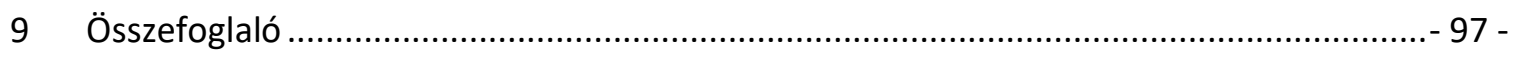

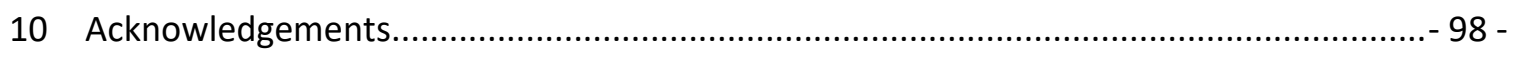

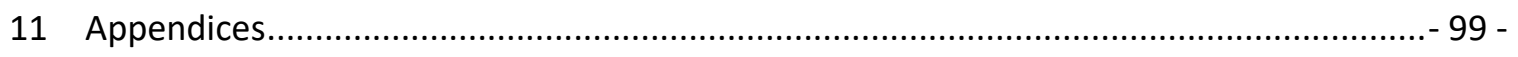

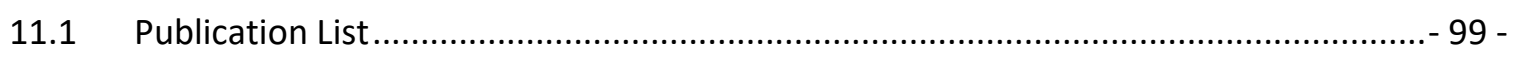




\section{List of abbreviations}

AML: Acute myeloid leukemia

ARF1: ADP ribosylation factor 1

ATP: Adenosine triphosphate

AKT: Protein Kinase B

BA: Benzyl Alcohol

Cyst: Cysteine

DMSO: Dimetil sulfoxide

DSC: Differential scanning calorimetry

DNA: Deoxyribonucleic Acid

ECM: Extracellular Matrix

GTPase: Guanosine Triphosphate

GM1: monosialotetrahexosylganglioside

GST: Glutation s transferase

GAP: GTPase Activating Protein

GDI: Guanine nucleotide Dissociation Inhibitor

GDP: Guanosine Diphosphate

GEF: Guanine nucleotide Exchange Factor

GSK3: Glycogen Synthase Kinase 3

GTP: Guanosine Triphosphate

GP: General polarization

HIV: Human immunodeficiency virus

HSP: Heat Shock Protein

IC50: half maximal inhibitory concentration

INTG: integrin

IQGAP1: Q Motif Containing GTPase Activating Protein 1

kDa: kilo Dalton

MEF: Mouse Embryonic Fibroblast 
mTOR: mammalian target of Rapamycin

NF-K $\beta$ : nuclear factor kappa-light-chain-enhancer of activated B cells

NMR: Nuclear magnetic resonance

PMA: phorbol 12-myristate 13-acetate

PI(4)P: phosphatidylinositol (4)- phosphate

$\mathrm{PI}(4,5) \mathrm{P}_{2}$ : phosphatidylinositol (4,5)-bisphosphate

PP2A: Protein phosphatase A2

PAGE: Polyacrilamide gelelectrophoresis

PVDF: Polyvinylidene fluoride

PRL: Phosphatase of regenerating liver

PAK: p21-activated Kinase

PBS: Phosphate Buffered Saline

PCBP1: poly C-binding protein 1

PI3K: Phosphoinositide 3 Kinase

Rac1: Ras related C3 botulinum toxin substrate1

RNA: Ribonucleic Acid

sHSP: Small Heat Shock Protein

SDS: Sodium dodecyl sulfate

SRC: sarcoma tyrosine-protein kinase

STAT: signal transducer and activator of transcription

Tyr: Tyrosine

TRPV: transient receptor potential vanilloid

TGF $\beta$ : tumor growth factor beta

Thr: Threonine

USP4: Ubiquitin-specific protease 4

VEGF: Vascular endothelial growth factor

5'-UTR: 5 unturned repeat 


\section{Introduction}

\subsection{The composition and organization of the plasma membrane}

All cells are surrounded by membranes. From the earliest times of cellular organization membranes defined the boundary between intracellular and extracellular space, thus conferring the cell with its own identity. Based on the chemical nature of the composing lipids, the membrane basic structure is the bilayer. The ability of the hydrophobic residues of the lipids to self-associate and the tendency of their hydrophilic moieties to interact with aqueous environments or with each other give the physical basis of this structure ${ }^{1}$. In an eukaryotic membrane, lipids are sterols, sphingolipids and glycerophospholipids ${ }^{2}$. A mammalian cell mainly contains cholesterol as sterol, but in contrast one can find a huge variety of lipids from the other two classes. Glycerophospholipids, the major structural lipid class, could vary in their head group, in their fatty acid chain length or saturation and also in the bonds linking the hydrocarbon chain to the glycerol. Some members of this group are the phosphatidylinositols, phosphatidylcholines, phosphatidylethanolamines, phosphatidylserines, phosphatidic acid and the plasmalogens ${ }^{3}$. The sphingolipids are also showing a great potential for creating a diverse set of lipids by the use of different ceramide backbones and by the addition of hundreds of various carbohydrate structures to their head group, while creating a glycosphingolipid molecule ${ }^{4}$. However, cell membranes are not solely composed of lipids, they also contain proteins spanning the bilayer or interacting with the lipids on the sides of the leaflet. The earliest model depicting the membrane as a uniform mixture of lipids with randomly floating proteins in it was described by Singer and Nicholson ${ }^{5}$. However, it has rapidly became clear that the membrane structure is far from random and displays an unimagined complexity. The discovery of non-bilayer membranes with biological significance ${ }^{6}$ and the fact that both the membrane proteins and the lipid bilayer show an actively maintained compositional asymmetry ${ }^{7}$ were key to this realization. During the next decade of membrane research it has also become clear that the lipid composition of the membrane is favoring the assembly of complex membrane structures. These studies have shown that cellular organisms use an enormous variety of lipids to form membranes, despite the fact that theoretically only one lipid would be able to form a lipid bilayer ${ }^{2}$. The question of which came first: the diversity of lipids or the 
high complexity of the membrane structure is almost as complex as the classical chickenegg dilemma. One possible explanation is that certain variety of lipids could have originated for the reason to survive better within harsh conditions, as a membrane assembled from many lipids could sustain a wider variety of environmental conditions ${ }^{2}$. Therefore a certain diversity of lipids at one point might have been reached by an organism in order to buffer external perturbations. Another clue, pointing towards the evolution of lipid diversity, is that protein-lipid interactions could result in holes in the membrane and these holes are filled in by specific lipids. Therefore with the evolutionary innovation of a new membrane interacting protein came the need for a lipid that could stuff the protein in the membrane ${ }^{8}$. The diversity of lipid classes is in good correspondence with the evolution of higher organisms as sterols and sphingolipids are only present in eukaryotic cells. The appearance of these two lipid classes can be considered a significant game changer in the management of cellular organization as the preferential association of sterols and sphingolipids with certain proteins provided the potential for lateral segregation within the membrane. This segregation has opened up a new field for phenotypic diversity within a cell, what can be best described by the appearance of several membrane trafficking events in the eukaryotic cells ${ }^{2}$. All of these studies, especially the ones aimed to describe the mechanism of molecular movements within the bilayer, made us to reevaluate our models describing the organization of the plasma membrane. Consequently, the observation of restrictions in the lateral mobility for both lipid and protein components within the membrane, has opened new avenues of research ${ }^{9}$. The dynamic subdomain formation was described to originate from several processes, like the binding of membrane receptors to the extracellular matrix, by the intracellular activation of receptors, by receptor-ligand interactions or by the interaction between membrane proteins with the cytoskeleton. The latter interaction with cytoskeletal elements could even result in the formation of "cytoskeletal fences" which could greatly modulated the free movement of membrane components ${ }^{9}$. The discovery of these dynamic, mostly actin based regulations of membrane laterality has led to the development of the picket fence concept of the plasma membrane ${ }^{10}$. Nonetheless some of the studies analyzing the formation of membrane subdomains have come to the conclusion that these nanostructures could be formed by their own under favoring biochemical composition of lipids and proteins ${ }^{11}$. In the following years the research on lipid lateral segregation and phase behavior in model membranes ${ }^{12,13}$ had met with the studies concentrating more on the biological side of membrane micro heterogeneity ${ }^{14,15}$ which led to the birth of the term lipid raft. Primarily the term raft was referring to a 
membrane microdomain enriched in cholesterol, sphingolipids and glycolipids ${ }^{14}$. Over time the concept got more developed and the observation regarding lipid rafts has been synthetized to the definition stating that lipid rafts are small $(10-200 \mathrm{~nm})$, highly dynamic, detergent resistant, heterogeneous, sterol- and sphingolipid-enriched domains in the plasma membrane that compartmentalize cellular processes ${ }^{15}$.

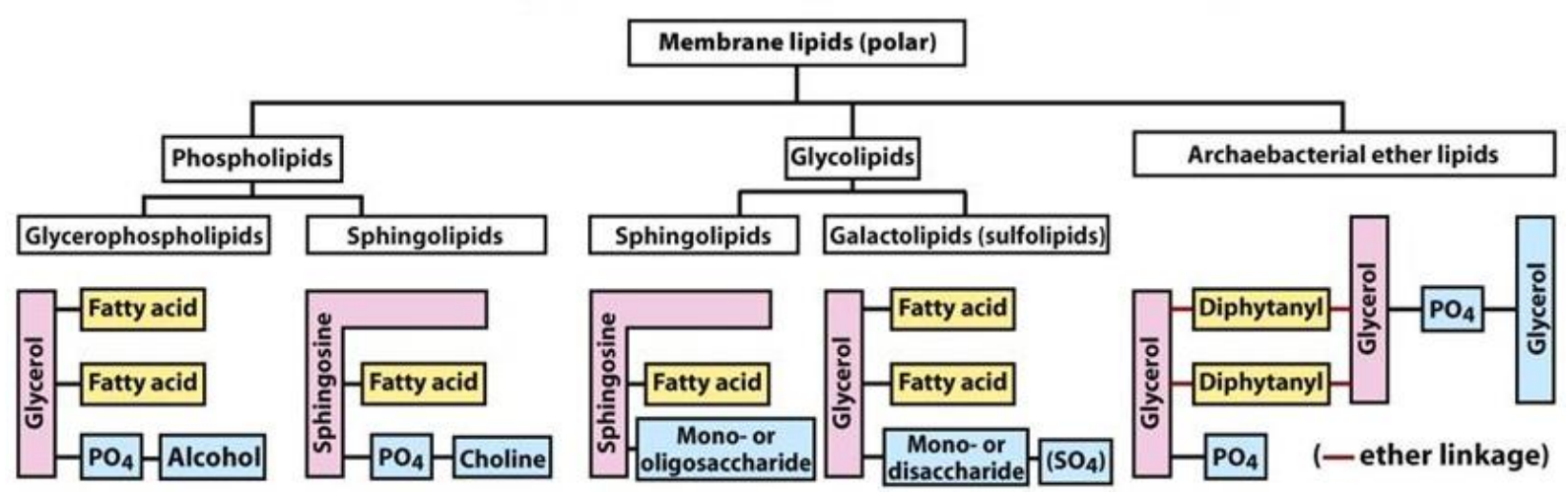

Table 1. Summary of the structural polar lipids in the plasma membrane (modified from Lehninger Principles of Biochemistry, Fifth Edition 2008)

\subsection{Membrane microdomains and the dynamic organization of the membrane}

Plasma membrane heterogeneity and compartmentalization occurs through lipid-lipid, lipid-protein, and membrane-cytoskeletal interactions. From all the lipids building up the membrane, cholesterol and sphingomyelin proportions are detected to be significantly higher in the raft structures compared to the overall plasma membrane and therefore are proposed to be essential components of these liquid ordered assemblies ${ }^{16}$. Saturated fatty acids of sphingomyelin are characteristically aggregate with cholesterol via an interaction with the saturated hydrocarbon chains and the sterol ring. Other lipids as ceramides could also be found in lipid rafts and are able to induce raft formation itself. Rafts are diverse in size but they are usually defined as small (10-200 nm) membrane domains. Although, under certain conditions small lipid rafts can coalesce to form larger and more stable platforms through protein-protein or protein-lipid interactions ${ }^{17}$.

Functionally lipid rafts are known to be involved in a broad range of cellular processes like membrane trafficking, cytoskeletal organization, cholesterol transport, endocytosis, 
cell polarity, motility and signal transduction ${ }^{18,19}$. Lipid rafts are central orchestrators of signal transduction as they provide platforms for the assembly of signaling complexes based on their compartmentalized nature. By sequestering signaling components, like receptors, they are allowing efficient and organized signal transduction to happen. Compartmentalization can allow the precise and timely regulation of receptor activation by confining them next to each other with their activators and interaction partners. Or on the other hand, a close negative regulation could be hold over a protein by keeping it apart from its kinases and close to their phosphatases ${ }^{17}$.

The proper organization of rafts is not only defined by their lipids but by their specific protein components and also by their link to the cytoskeleton as well. There are two main types of raft structures within the membrane: planar (flat) and non-planar (non-flat). Planar rafts lack any morphological feature in contrast to the non-planar structures which are classically defined as invaginated flask like structures of the membrane, usually known as caveolae. Both raft types are similar in their nature of being resistant to extraction by cold non-ionic detergents. Another feature of rafts is that they contain raft resident proteins which can provide important scaffolding functions, allowing the sequestration of signal proteins into the raft fraction. In planar rafts typically flotillins are serving this function and in non-planar rafts caveolin is the scaffold most frequently present ${ }^{16,20}$.

Beside the scaffolding interactions many signaling proteins are dependent on their covalent posttranslational lipid modification for their targeting to lipid rafts. Glycosylphosphatidylinositol, an anchor targeting proteins to the extracellular surface of lipid rafts, was the first to be discovered from these molecules. That was followed by the description of other protein modifications by lipids, as prenylation, palmitoylation, $\mathrm{N}$ myristoylation and dual acylation which target the proteins to the cytoplasmic side of the plasma membrane ${ }^{2,21}$. 


\subsection{Caveolae and their role in signaling compartmentalization}

As was mentioned earlier, the first rafts structures were discovered by transmission electron microscopy studies by detecting cave like invaginations in the plasma membrane. The name caveolae is also originating from these observations ${ }^{18}$. Later studies further characterized the caveolae as relatively large, 50-100 nm long formations which can be organized into complex highly ordered multi-caveolar structures or dynamically detach and re-attach to the membrane while keeping their defined lipid and protein composition intact. The latter process was termed as caveolae mediated endocytosis. The amount of caveolar structures within a cell is highly heterogeneous and dependent on the tissue type and micro-environmental conditions. The highest abundancy of caveolae is detected in muscle tissues, endothelial cells, adipocytes and fibroblasts but the absolute absence of caveolae is rare within physiological conditions ${ }^{22}$.

The two major structural caveolae proteins are the caveolins and the cavins, both are required for the formation of caveolae. The caveolins are acting as the major scaffold within the caveolae, thereby responsible for the management of protein-protein interactions ${ }^{18}$. Caveolins are also lipid binding proteins, they can form stable complexes with cholesterol within the endoplasmic reticulum. These complexes are shuttled together to the plasma membrane in the process of caveolae formation. Therefore, cholesterol is essential in the development of caveolae. Since of its critical importance, a direct transcriptional regulation can be observed over the expression of caveolins via a cholesterol sensitive steroid binding element in their promoter region ${ }^{23}$. Caveolins have both their $\mathrm{C}$ - and $\mathrm{N}$-terminal end facing towards the cytoplasm and are also capable of forming large homo-oligomeric and hetero-oligomeric complexes that allow their selfassembly into caveolae. The caveolin family consists of three proteins caveolin 1 , caveolin 2 and caveolin 3 . From these, caveolin 1 shows the widest tissue distribution and usually accepted as the general caveolin protein within the caveolae. Caveolin 2 is usually co-expressed with caveolin 1 and is unable to form caveolae in the absence of caveolin 1 . Caveolin 3 is shown to be specific only to muscle tissues ${ }^{24}$. The cavin proteins are the other essential components of caveolae, serving as primary modulators of its biogenesis, morphology and endocytic dynamic ${ }^{25}$. The cavin family consists of four members which tend to self-aggregate into hetero oligomeric structures ${ }^{26}$. Among these cavin 1 is generally expressed, cavin 2 is usually detected in enlarged tubular like caveolar multi-complexes while cavin 3 is suspected to play a role in the budding and 
movement of the caveolae along the cytoskeleton. Our knowledge on the role of cavin 4 is very limited as it has only been detected in muscle tissues ${ }^{26}$. Cavin is also capable of lipid binding, specifically to phosphatidylserine which is enriched in the inner leaflet of caveolin rich membrane regions. This interaction is hypothesized to help the formation of the classical non-planar flask like structure of the caveolae from the planar caveolin and cholesterol rich membrane regions ${ }^{25}$. Both caveolins and cavins are indispensable for caveolae formation as their genetic ablation causes the total loss of caveolae ${ }^{27}$.

Caveolae could exert many functions within a cell. They are involved in intracellular lipid regulation and transport, based on the ability of caveolins to associate with cholesterol and fatty acids ${ }^{27}$. The caveolae could also act as a mechanosensor, extensive mechanical stimulation can result in the flattening of caveolae within the plane of the membrane in the short term ${ }^{28}$ and the increase of caveolae numbers in the long term ${ }^{29}$. Therefore caveolae can act as membrane reservoirs against mechanical stresses-stretches. However, the most well described role of caveolae is its regulatory function over cellular signaling. The dynamic distribution of caveolae is suggested to contribute to the progression through the cell cycle by regulating the distribution of plasma membrane micro domains during mitosis ${ }^{30}$. Another mechanism of how caveolae controls cell division is its dynamic regulation over the integrin $\beta 1 /$ Rac1 pathway. Upon attachment to a substrate, integrin signals regulate the levels of cyclin D1, a key cell cycle regulator of the G1/S transition. However, when the integrin signal is aborted the cell initiates the endocytosis of the caveolae resident integrin/Rac1 complex in order to physically disconnect it from their effectors, thereby shutting down the growth signal originating from these proteins. This phenomena is suggested to act as a fast acting regulatory switch against uncontrolled cell division ${ }^{31,32}$. The precise modulatory role of caveolae over the cell cycle is further demonstrated by the fact that the forced overexpression of caveolin 1 is observed to results in a distinct growth arrest ${ }^{33}$. Caveolae like structures were also shown to play a fine tuning role in the regulation of the mitotic spindle assembly by transmitting signals from the retracting edge of the cell ${ }^{34}$. Caveolae also participates in the fine regulation of tissue repair and differentiation by sequestering key signaling molecules like $\beta$-catenin or the transforming growth factor $\beta^{35}$. 


\subsection{Membrane microdomains in cellular signaling: the role of membrane fluidity and lateral domain mobility in cellular stress sensing}

Because of being the outermost boundary of the cell, the plasma membrane is deeply involved in the sensing and transmission of signals from the environment towards the cellular interior. As we have seen from the previous chapters, lipid raft structures are fundamentally different from their surrounding membrane environment which allows them to act as organizing hubs. The following part of the thesis is going to discuss an intriguing example of such function of the membrane, namely the involvement of lipid rafts in the organization of environmental stress sensing. Detailing particularly the phenomena of how changes in the membrane structure can define cellular behavior, gene expression and survival under stress conditions.

\subsubsection{Chaperones and the membrane-mediated stress response}

All living organisms can be subjected to environmentally-imposed challenges. In order to survive they have to counter these unfavorable conditions by adaptation. Responsiveness to diverse stressors may arise from the most striking and common impact of stress: it deforms and may damage the cell constituent molecules such as membrane lipids, proteins or nucleic acids ${ }^{36}$. The term cellular stress response is summarizing the universal cellular reaction to the wide variety of environmental challenges ${ }^{37}$. Among the many cellular survival mechanisms the response to heat stress is amongst the most ancient and conserved ones ${ }^{38}$. Heat stress induces a multi-leveled signaling in cellular systems termed as the heat shock response. The heat shock response is originally defined as the biochemical response of cells to heat stress ${ }^{39}$. Although, it has been primarily described to be induced by increased temperatures, it has been later recognized that the heat shock response can be activated by a wider range of cellular stressors like heavy metals, oxidative stress or by pathophysiological conditions ${ }^{40}$. One of the most characterized aspects of the heat shock response is the accumulation of a conserved set of proteins called heat shock proteins (HSPs). Heat shock proteins act as chaperones within the cell by aiding the reassembly and refolding of denatured proteins and by guiding the degradation of irreversibly damaged proteins. Therefore the HSPs are involved in the maintenance of protein homeostasis by alleviating the unfavorable changes caused by 
heat stress ${ }^{41}$. The accumulation of heat shock proteins is generally leading to an elevated level of cellular resistance, known as "thermotolerance". HSPs however, are described to have a larger range of possible action besides acting as molecular chaperones having multiply functions within a cell depending on their location. HSP chaperones are consisting of many different families differentiated by their molecular weight.

The small heat shock proteins (sHSPs) are the most diverse set of HSPs, consisting of proteins of 12-43 $\mathrm{kDa}$ range. They can form large multimeric complexes and display a wide range of cellular functions. The sHSPs coaggregate with aggregation prone proteins in order to initiate their efficient disaggregation. The release of substrate proteins from the transient sHSP reservoirs and their refolding require cooperation with ATP-dependent chaperone systems ${ }^{42}$. The sHSP holdase, $\alpha$-crystallin can behave as a chaperone-like protein sequestering unfolded proteins, and inhibiting subsequent aggregation and insolubilization in the eye lens ${ }^{43}$. Mouse HSP25 and human HSP27 can form large oligomeric structures which can act as molecular chaperones and protect cells from heat shock and oxidative stress and affect the development of thermotolerance. HSP25/27 is additionally involved in actin polymerization/depolymerization ${ }^{44}$. HSP60 is an ATPdependent unfoldase. The HSP60 proteins in eukaryotes are localized to the mitochondria and are responsible for mitochondrial protein import and macromolecular assembly within this compartment ${ }^{45}$. HSP70 is most likely the best characterized chaperone. The HSP70 proteins are acting together with their aids the HSP40 proteins. HSP40 proteins are showing a great diversity and can take part in the substrate specificity of HSP70, and can increase the ATPase activity of the HSP70 protein thereby enhancing their activity. The HSP70/HSP40 system participates in the active ATP dependent folding-refolding of a high number of cellular proteins. Although, a precise mechanism has yet to be determined, it is known that the HSP70s have a high-affinity for unfolded proteins when bound to ADP, and a low-affinity when bound to ATP ${ }^{46}$. The molecular chaperone HSP90 is also an ATP-dependent holdase that facilitates the maturation and/or activation of over 100 'client proteins' involved in signal transduction and transcriptional regulation and is necessary for viability in eukaryotes. HSP90 is central to processes broadly ranging from cell cycle regulation to protein degradation and it has been also connected with cellular transformation ${ }^{47}$. The proteins in the HSP100 family form large hexameric structures with unfoldase activity in the presence of ATP. These proteins are considered to function as chaperones by recessively threading client proteins through a small pore, thereby providing each client protein with a second chance to fold ${ }^{48}$. 
Major HSPs and their actions can be seen in Table 2.

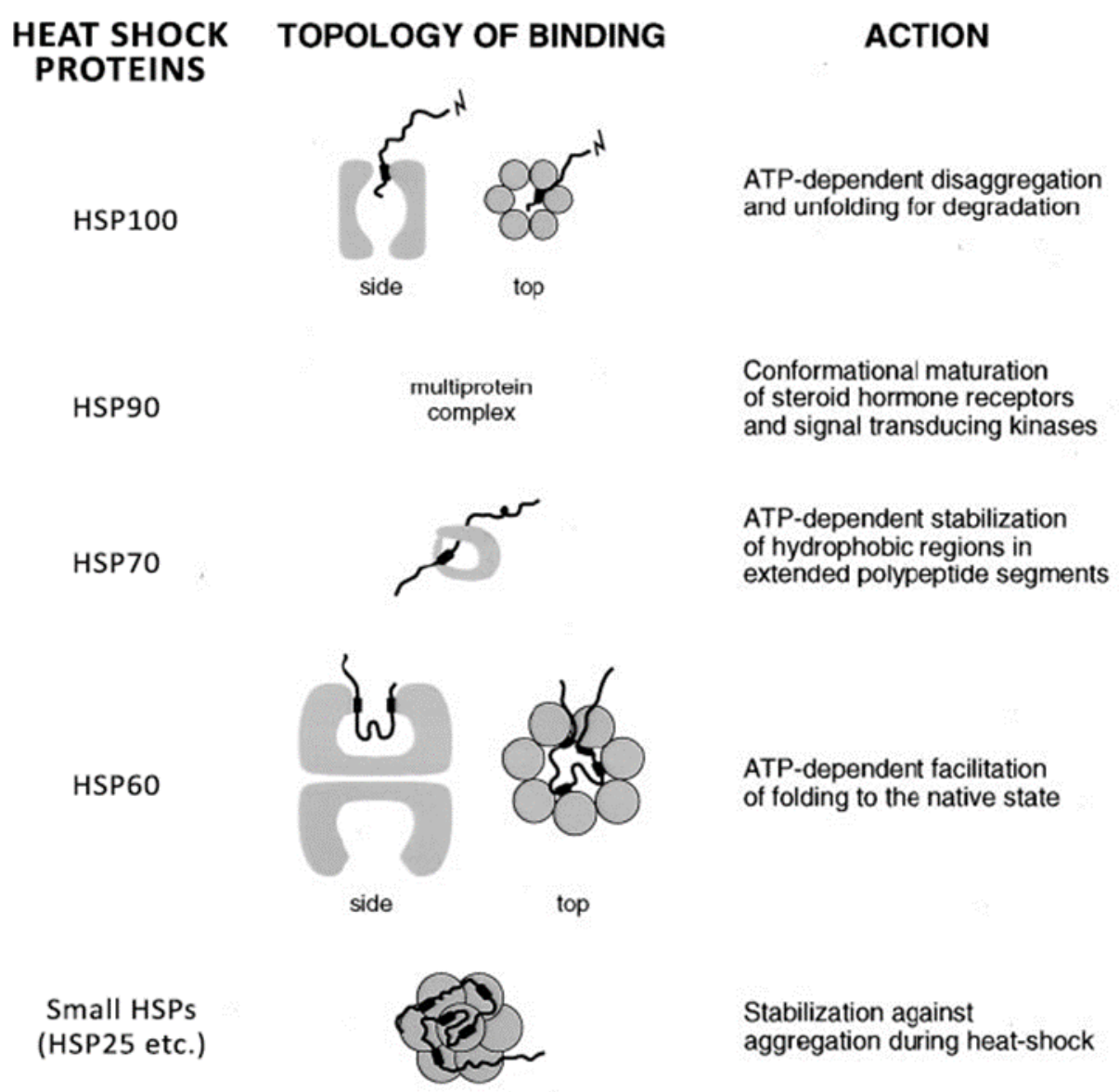

Table 2. Topology of Polypeptide Binding and Action of HSP Families. Bold lines signify polypeptides, and the thickened segments denote sites that become directly associated with chaperone, typically hydrophobic in character. (Bukau \& Horwich 1998).

Originally the heat shock response and the induction of HSP expression was described to be primarily activated by protein denaturation and aggregation. Besides this classical model, it has been recognized that cells can sense the heat stress in different ways. The membrane sensor model of heat sensing describes one of these mechanisms. It emphasizes the importance of the plasma membrane, especially its microdomain organization, in the sensing of heat stress and in the activation of the heat shock response 49. The exposure of a cell to elevated heat conditions alters the physical state of the plasma membrane by fluidizing the membrane. It was shown decades ago that the fluidity, organization and phase behavior of membranes are key factors in the processes of thermally-induced adaptation and cell survival, in both prokaryotic ${ }^{50}$ and eukaryotic 
cells ${ }^{51}$. Early findings revealed that membranes can act as thermosensors and there exists a membrane fluidity based feed-back control over certain stress responsive genes like fatty acid desaturases and heat shock proteins ${ }^{52,53}$. But the question of how the temperature caused structural plasma membrane alterations are perceived and translated into gene expression changes still remains largely unknown. Biochemical and cell biological studies have prompted that lipid rafts play a crucial role in many signaling processes including stress sensing and adaptation ${ }^{54}$. Early, pioneering studies showed that the membrane's physical state could influence gene expression and hence, responses to stress ${ }^{55}$. Since then, our knowledge of the interactions between the plasma membrane and the gene-expression machinery has increased significantly as our understanding of membrane microdomains has widened ${ }^{56}$. It has become clear that since the raft structure is strongly dependent on thermally-controlled lipid-phase behavior, changes in temperature could result in altered fluidity and consequently, redistribution of the membrane domains. This change in the lateral segregation behavior of membrane domains has been shown to play a central role in heat stress sensing and the initiation of the heat shock response. The universal induction of cell stress machineries in various organisms (bacteria, algae, yeast, plant and mammalian cells), by the treatment with nonproteotoxic membrane fluidizers strongly suggests, that subtle changes in the surface membranes are key upstream events of the temperature-sensing and signaling. The reorganization of membrane domains upon heat was further connected with subtle changes in the underlying actin network.

Heat shock genes were one of the first detected genes to be affected by perturbations in the membrane structure ${ }^{57}$. Thus the protein- and/or lipid-mediated association of a specific set of stress protein molecular chaperones to membranes is a well-known phenomenon, and is implicated to play a role in a number of physiological and pathological events ${ }^{58}$. Temporary association of certain HSPs with membranes can reduce the level of fluidity ${ }^{59}$, elevate bilayer stability ${ }^{60}$ and thereby restore the membrane functionality during heat stress conditions. The association with the plasma membrane seems to account for the pleiotropic effect of HSPs (predominantly small HSPs) which can contribute to the restoration of membrane activity following damage caused by abiotic stresses. It is hypothesized that a subset of sHSPs functions in the cellular stress management by acting as membrane-stabilizing factors. It is suggested that sHSP-mediated membrane stabilization precedes the thermal adaptation that occurs by adjustment of the lipid composition ${ }^{59}$. The fluidity and microdomain organization of 
membranes are decisive factors in the perception and transduction of stresses into signals that trigger the activation of specific heat shock genes ${ }^{51}$. Conversely, the membrane association of specific HSPs may result in the inactivation of membrane-perturbing signals, thereby switch off the heat shock response. In that context, interactions between certain HSPs and specific lipid molecular species might be a previously unrecognized means for the compartmentalization of HSPs to specific signaling platforms, where key stress signaling proteins are known to be concentrated.

\subsubsection{The association of small heat shock proteins with membranes}

The earliest finding describing the connection between membranes and sHSPs was concentrating on the $15 \mathrm{kDa} \mathrm{HSP}$ of Escherichia coli since it was observed to cosediment with membranes. The subsequent studies further characterizing this protein, came to the conclusion that in fact this were a hetero-association of two proteins which is known as IbpA and IbpB since then ${ }^{61}$. Further evidence came from the studies concentrating on the hsp17 gene of Synechocystis PCC 6803, a blue-green algae, as the transcription of the hsp17 gene was strongly regulated by changes in the physical order of the membrane ${ }^{49}$. Another example, reinforcing the association of sHSPs with membranes, was coming from a lactic acid bacterium Oenococcus oeni as the expression of one of its sHSPs, termed Lo18, was induced by the administration of the membrane fluidizer benzyl alcohol. As benzyl alcohol treatment mimics the fluidizing effect of heat on the membrane, it can be interpreted as a model of heat induced membrane fluidization. It has further been demonstrated that a subset of Lo18 is localized in the membrane fraction and the actual level of its membrane association was dependent on the temperature ${ }^{62}$. HSP16, a sHSP from Mycobacterium tuberculosis was also found to be a major membrane protein ${ }^{63}$. These observations laid the groundwork for the discovery of membrane localized sHSPs in eukaryotic systems and thus to the identification of the membrane association of sHSPs in mammalian cells. In the following years the list of membrane localized sHSPs has broadened quickly, as HSPB2, expressed in heart and skeletal muscle, were shown to associate with the outer membrane of the mitochondria ${ }^{64}$. A similar observation has been made with the major protein component of the vertebrate eye lens $\alpha$-crystallin, which was shown to play a central role in the maintenance of lens transparency through its ability to inhibit stress-induced protein aggregation ${ }^{65}$. A novel 
16.2 $\mathrm{kDa}$ human sHSP, HspB11, was described to inhibit $\mathrm{H}_{2} \mathrm{O}_{2}$, taxol or etoposide induced cell death through preserving the integrity of the mitochondrial membrane system and by the stabilization of plasma membrane lipid rafts ${ }^{66,67}$. These studies proved for some sHSPs, that they differentially interact with membrane lipids and the interaction influences membrane physical properties. The biophysical-biochemical role for sHSPs in the control of the physical state, bilayer stability and integrity of membranes via specific lipid interactions has been established by studying the HSP17 of Synechocystis PCC 6803, in which most of the heat-induced HSP17 is associated with thylakoid membranes 49. As evidenced by in vivo genetic and in vitro biochemical studies, HSP17 is able to play a fundamental role in membrane quality control and thereby it is potentially contributing to the maintenance of membrane integrity under stress conditions ${ }^{59}$. Thus, HSP17 possesses not only protein-protective activity, but also a previously unrecognized ability to stabilize the lipid phase of the membranes ${ }^{68}$. The interactions between purified HSP17 and large unilamellar vesicles, consisting of synthetic or cyanobacterial lipids, strongly increase the microviscosity of the vesicles ${ }^{59}$. Another member of the family of the sHSPs, $\alpha$-crystallin was also shown to have stabilizing effects on model membranes, as $\alpha$-crystallin were able to stabilize the liquid-crystalline state in anionic membranes formed from synthetic lipids ${ }^{68}$. In membranes composed of the non-bilayer lipid dielaidoyl-phosphatidylethanolamine, DSC studies revealed that, even at an extremely low protein:lipid molar ratio, HSP17 strongly stabilizes the lamellar liquid-crystalline phase at the expense of the non-lamellar lipid phase ${ }^{68}$. This suggests that sHSPs can modulate membrane lipid polymorphism. In membranes composed of monogalactosyldiacylglycerol and phosphatidylglycerol (both enriched with unsaturated fatty acids) isolated from Synechocystis thylakoids, HSP17 and $\alpha$-crystallin increase the molecular order in the fluid-like state. This data shows that the nature of sHsp-membrane interactions depends on the lipid composition and extent of lipid unsaturation, and that sHSPs can regulate membrane fluidity ${ }^{59}$. It can be hypothesized from these results that the association between sHSPs and membranes may constitute a general mechanism that preserves membrane integrity during thermal fluctuations. According to these studies HSP17 behaves as an amphitropic protein and plays a dual role. Depending on its membrane or cytosolic localization, it may function as a membrane-stabilizing factor and/or as a member of a chaperone protein-folding network. It should be noted that a similar observation has been made by studying the sHSP Lo18 and its lipid interaction with the subsequent fluidity changes caused by it ${ }^{62}$. Lo18 was shown to interact with phospholipids and the association of Lo18 with liposomes formed from purified total 
lipids of Oenococcus oeni membranes reduced membrane fluidity at elevated temperatures. This phenomenon was obtained by the general polarization measurements with the membrane incorporating Laurdan dye which showed that Lo18 maintained phospholipids in a higher lipid order. Thus, Lo18 can modulate membrane lipid properties by direct action on phospholipids ${ }^{62}$. Since the lipid specificity observed in the case of HSP17 and Lo18 has also been documented for $\alpha$-crystallin it is tempting to hypothesize that the membrane binding of small HSPs through specific lipid interactions may confine the location of the sHSPs to specific (raft like) membrane lipid domains in mammalian systems ${ }^{59}$.

The above detailed studies are reinforcing the hypothesis that membrane organizational changes are key to the initiation of the heat shock response and lipid-associated pool of sHSPs may play important roles in the protection of membranes under stress conditions. The data detailed above strongly implies that membrane association of sHSPs antagonizes the heat-induced fluidization and is simultaneously able to prevent the formation of a membrane-disrupting non-bilayer lipid phase. Thus, they are central players in the maintenance of the membrane structure under damaging stress conditions. Furthermore, the lipid-mediated and transient association of sHSPs to specific membrane domains may also serve as part of a feedback mechanism in the regulation of heat shock genes. Although, these mechanisms are still under investigation, they are representing a potential sensing and signaling pathway of the cell which is utilized under stress conditions in order to balance out membrane lipid perturbation. 


\subsection{Membrane microdomains at the forefront of disease states: caveolae resident proteins as central players in cancer progression}

Lipid rafts are creating a great degree of membrane heterogeneity and by this they are allowing a higher level of compartmentalization to happen in the plasma membrane. This heterogeneity has a great significance in signaling processes at the membrane level, as the compartmentalized nature allows the lipid rafts to act like sorting platforms for signal transduction proteins. Many proteins assembled in lipid rafts are involved in a wide range of signaling processes, like cell survival, growth signaling, adhesion and migration. An important defining nature of these platforms is that they are highly dynamic and capable of rapid changes. It means in practical terms that these sorting structures of the membrane can react to rapid changes in the environment in a very short time and can re-establish their basal state after the loss of the signal in the same rapid way. Thus allowing a precise regulation over cellular signaling and a prone and timely response to environmental signals ${ }^{16}$. However, if this sensitive temporal and spatial regulation gets out of balance it could result in the occurrence of many pathophysiological states. In a case of dysregulated raft signaling these structures could act as scaffolding hubs where the segregation of pathological proteins take place ${ }^{56}$. Therefore rafts, especially caveolae and its resident proteins, can be critical in the development of a wide range of pathologies. For example caveolin 1 was shown to mediate the initiation of the atherosclerotic plaques during the development of atherosclerosis ${ }^{69}$. A group of skeletal muscle diseases were even grouped and termed as caveolinopathies in connection with the common mutational background in the caveolin 3 gene ${ }^{70}$. Caveolin deficiency has also been connected with cardiac dysfunction and was shown to worsen the outcome of myocardial infarction ${ }^{71}$. Caveolae and the protein content of caveolae was observed to play a role especially in the development and progression of cancer ${ }^{19}$. As it has been mentioned earlier caveolae plays a fine tuning, regulatory role in cell cycle signaling and in the initiation of growth arrest upon non adherent conditions ${ }^{30,72}$. The dysfunction of this regulation results in continuous cell proliferation ${ }^{73}$. In the recent years therapies targeting such raft recruited proteins and the raft structures via modulation of the cell membrane by classical pharmacological means ${ }^{74}$ or by direct introduction of membrane altering lipids ${ }^{75,76}$ are becoming more and more relevant approaches in cancer therapy.

However, our knowledge on dysregulated raft resident proteins is scarce at the current moment. Therefore a stringent analysis of raft resident, disease associated proteins must 
take place in order to design disease specific treatments in the future. One approach to this is to analyze known disease specific proteins in their possible membrane-raft context as an initial step to uncover their precise action. Such clarification would greatly help the design of future therapies by unravelling a critical feature of the disease.

In the course of the next chapter, the thesis is trying to give a comprehensive background for the research work detailed in the results and discussions part by concentrating on a known membrane associated oncogene. This protein has been central to the work of the thesis, the major aim of which was to understand its mechanism of action in the context of its association to the plasma membrane. Thus, with the analysis of this protein, this doctoral work is trying to apply the approach mentioned above by analyzing disease causing proteins within their membrane context.

\subsubsection{A group of membrane anchored oncogenes: the PRL family of dual specificity phosphatases}

The phosphatase of regenerating liver (PRL) family of dual specificity phosphatases were described first during the analysis of regenerating liver tissues following liver injury whereas PRL1, the first member of the family showed a distinct expression ${ }^{77}$. The two other members (PRL2 and PRL3) were discovered by searching for sequence similarities in an expressed sequence tag dataset ${ }^{78}$.

Structurally, the three human PRLs have a 75-87\% amino acid sequence homology and all three proteins have molecular weights of about $20 \mathrm{kDa}{ }^{79}$. This similarity is represented in other species as compared to murine PRLs: PRL1 and PRL2 is 100\% homologous and only mouse PRL3 shows a 97\% sequence identity compared to human PRL3 ${ }^{80}$. However, despite the high similarity between its own family members the amino acid sequence of the PRLs does not overlap with other phosphatases except the common catalytic phosphatase motif ${ }^{81}$. The highest identity they share among the group of dual specificity phosphatases is with the phosphatase and tensin homolog protein which is around $17 \%{ }^{80}$. The three dimensional solution structure with and without of a phosphate group at the catalytic center has been solved by NMR for PRLs ${ }^{82}$. These studies were showing a highly flexible active loop for the PRLs with a special architecture allowing them to bind phospho-serine phospho-threonine phospho-tyrosine residues, a characteristic feature for dual specific phosphatases ${ }^{82}$. A high variety could be 
observed around the opposite side of the conserved catalytic pocket that is hypothesized to play a role in substrate specificity ${ }^{81}$. Cys104 was detected to serve as a nucleophile in the substrate hydrolysis ${ }^{81}$ which is regulated by the Cys 49 residue as under high oxidative conditions it forms a disulfide bond with the catalytic Cys $104^{81}$. It is postulated that this bond formation is necessary to protect the catalytic core of PRLs against excessive oxidation ${ }^{83}$. Furthermore PRL1 has also been detected to be increased under high oxidative conditions suggesting a role for the PRL proteins in the defense against oxidative stresses ${ }^{84}$. Thioredoxi-related protein 32 was observed to regulate the reduction of PRL3 and therefore its catalytic activity under oxidative stress ${ }^{83}$ thereby pinpointing a key regulator of PRL3's biological function. Both PRL1 and PRL3 has been detected to form trimers both in vitro and within the cell, which is necessary for the proteins to acts as an oncogene in cancer cells ${ }^{85}$.

The three PRLs have a posttranslational modification CAAX motif for prenylation required for the association with the plasma membrane. This prenylation motif is unique to the PRLs within the group of dual specificity and the tyrosine phosphatases ${ }^{86}$. During protein prenylation a farnesyl (C15) or a geranylgeranyl (C20) group is attached to the Cterminal end cysteine of a protein. The process of prenylation first requires the attachment of the lipid group to the CAAX motif by a farnesyl-transferase, followed by the cleavage of the last three AAX amino acid by a prenyl-CAAX protease, then as a final step the carboxyl group of the prenylated cysteine is methylated by a prenylcystein carboxyl-methyltransferase ${ }^{87}$. The in vitro analysis of PRLs proved that both PRL1 and PRL2 are farnesylated and geranylgeranylated as well, but PRL3 only shows farnesylation ${ }^{88}$. Surprisingly the deletion of PRL3's CAAX motif resulted in the increase of its catalytic activity and decrease in their trimer formation ${ }^{85}$, suggesting a role for the CAAX motif in the trimerization and implying a presence of a precise regulator in the plasma membrane that affects PRL3's catalytic activity.

Beside the protein prenylation an C-terminal polybasic amino acid stretch was shown to be also necessary for the membrane localization of the PRLs ${ }^{85}$. The six basic amino acid residue located in close proximity to the CAAX motif was also detected to aid the correct membrane localization of the PRLs by interacting with the negatively charged phospholipids of the membrane ${ }^{85}$. This proposed function for aiding the precise sublocalization of PRLs within the membrane is emphasized by the study which showed that deletion of the amino acid stretch resulted in the loss of membrane localization, only if the CAAX motif was also mutated ${ }^{89}$. Such regulation of protein localization is not 
without a precedence. A similar stretch of basic amino acid residues was detected to play a role (together with their prenylation) in the correct membrane localization of the Ras and the Src proteins ${ }^{90}$.

\subsubsection{PRL3 in cancer}

The PRLs has been implicated to play a causative role in a broad range of cancerous processes ${ }^{91,92}$. A number of clinical evidences show that among the family members PRL3 has the strongest association with tumor progression ${ }^{79,93}$. In primary gastric tumor lesions the elevated level of PRL3 expression is associated with tumor growth and has a negative impact on the prognosis ${ }^{94,95}$. Furthermore, PRL3 is suggested to predict the relapse of gastric cancer ${ }^{96}$ and the potential reoccurrence of invasive breast cancer ${ }^{97,98}$. PRL3 has also been evaluated as a prognostic factor in hepatocellular carcinoma 99 ovarian ${ }^{100}$ and colon cancer ${ }^{101}$. Moreover PRL3 has been described as a predictor of metastasis if expressed in primary tumors ${ }^{102,103}$ and the antibody therapy targeting PRL3 has been successful in preventing tumor reoccurrence and prolonging animal survival after surgical tumor removal ${ }^{104}$. Initial observations described PRL3 as a one of the few genes that is highly expressed in colorectal cancer metastasizes ${ }^{91}$.

PRL3 has been hypothetised to effect tumor angiogenesis in a few studies 105-107. However, the exact mechanism is not described in detail, yet, in some cell lines as in chinese hamster ovary cells or in the DLD1 human colon carcinoma PRL3 expression mitigated andothelial cell recruitment towards the cells which is might effect tumor vascularisation in the long term ${ }^{105,106}$ what could be the basis of PRL3 associated tumor vascularisation. A possible mechanism was suggested by another study describing an increase in vascular endothelial growth factor expression in association with PRL3 expression in lung cancer cells which was hypothetysed to induce microvascular and lymphatic vessel formation ${ }^{107}$.

PRL3 has been revealed to effect tumor metastasis and migration in several model systems ${ }^{108,109}$. PRL3 has been linked to proteins regulating cellular adhesion, for example integrin $\beta 1$ was shown to be crucial for the PRL3 mediated induction of tumor migration in colorectal carcinoma cells ${ }^{110}$. Other plasma membran associated effectors of cell migration were also affiliated with PRL3, e.g. ezrin a key plasma membrane resident adaptor of the cytoskelton was shown to be dephosphorylated in the presence of PRL3 at 
its Thr567 site ${ }^{111}$. The phosphorylation of ezrin at Thr567 is a key event in the ezrin mediated membrane recruitment of F-actin and the subsequent recruiting of guanidine exchange factors (GEFs) regulators of the Rho family GTPases ${ }^{112}$. Therefore the dephosphorylation on this site could directly result in the imbalance in the activity of the Rho GTPases ${ }^{112}$ like Rac which might result in increased cell migration, as shown in previous studies in connection with PRL3 ${ }^{113}$. PRL3 was indicated to affect tumor dissemination by increasing the invasiveness of cancer cells ${ }^{110,114}$ either by enhancing the expression of a matrix metallo-protease which can help the release of cancer cells from their original environment ${ }^{110}$ or by affecting the expression of proteins directly responsible for cell motility ${ }^{114}$.

PRL3 has also been recognised to promote tumor proliferation and growth ${ }^{77}$. Cancer cells from different origins has been shown to grow in non-adherent conditions ${ }^{105,115-117}$ upon PRL3 expression. PRL3 has been implicated to play a crucial role in the regulation of ovarian cancer growth ${ }^{116}$. Cancer progression and tumor growth has also been shown to be affiliated with the aberrant activation of mTORC1 ${ }^{118}$ and the PI3K/AKT pathway ${ }^{118-120}$ induced upon PRL3 expression. Furthermore the growth promoting effect of PRL3 has markedly increased under stress conditions suggesting that the presence of PRL3 is a strategic advantage for cancer cells to survive in conditions where otherwise they would not ${ }^{118,120}$. PRL3 was also shown to induce the activation of the epidermal growth factor receptor and its downstream signalling that could also be associated with tumor growth ${ }^{119}$. Further evidence suggests a role for PRL3 mediated growth in therapeutic resistance since the inhibitor of PRL3 has been characterized as a potent adjuvant of cisplatin treatment ${ }^{121}$. Moreover PRL3 depletion sensitized acute myeloid leukemia (AML) cells to anti-cancer therapy ${ }^{122}$. The elevated cyclin D1 levels were hypothesized to play a contributory role for PRL3's promotion of AML growth ${ }^{122}$. The work on PRL3 knockout mouse models has led to the conclusion that PRL3 plays a critical role in primary tumor formation ${ }^{123}$ since mouse deficient in PRL3 has developed a significantly lower amount of colon tumors after treatment with a common carcinogen azoxymethane. The connection between tumor initiation and PRL3 was further pinpointed by a study showing high PRL3 expression in the CD133 positive tumor initiating cell population of colon carcinoma cells ${ }^{124}$. Furthermore, tumor initiating cells originating from a PRL3 knock-out mice were less prone to initiate tumor growth in mice ${ }^{124}$. The validity of this association was emphasized by another study which recognized a relation between PRL3 expression and stem cell like behavior in AML cells ${ }^{125}$. As this study describes, PRL3 
expression maintains leukemogenesis both in in vitro and in vivo conditions by inducing a stem cell-like transcriptional program conferring stem cell-like properties to leukemia cells ${ }^{125}$. These results are not without previous evidence as the other member of the PRL family, PRL2 was shown to be important for hematopoietic stem cell self-renewal ${ }^{126}$ and for the maintenance of c-kit, the receptor of stem cell factor, in spermatogenesis ${ }^{127}$.

A surprising connection can be discovered between the phenomena of PRL3 induction upon several DNA damaging agents in non-cancerous MEF cells ${ }^{128}$ and the observed elevated PRL3 expression in carcinogen treatment induced colon cancer ${ }^{123}$. In the MEF cells the prolonged PRL3 expression induced Akt signaling resulted in a cell cycle arrest in contrast to cancer cell where PRL3 failed to trigger the same phenomenon ${ }^{128}$. Moreover, PRL3 was shown to be critical in the initiation of colon cancer from carcinogen treated mouse epithelia ${ }^{123}$. This suggests a complex regulation upstream PRL3 in non-cancerous tissues which might be lost or mutated during carcinogenesis.

Considering the overwhelming evidence for PRL3 in cancer progression compared to the other members of the family, the following parts of this chapter are concentrating on the description of this protein in a deeper detail.

\subsubsection{PRL3 associated regulation and signaling}

Several layers of regulation has been observed over PRL3 involving transcriptional, translational and proteosomal levels ${ }^{109,129-131}$. PRL3 was discovered to be a direct transcriptional target of P53 in non-cancerous MEF cells ${ }^{128}$ and further associated with other growth factors or transcription factors as the vascular endothelial growth factor ${ }^{132}$, the myocyte enhancer factor ${ }^{132}$, the transforming growth factor $\beta$ (TGF $\left.\beta\right)^{120}$, nuclear factor kappa-light-chain-enhancer of activated B cells $(\mathrm{NF}-\mathrm{\kappa B}){ }^{133}$, the transcriptional factor SNAIL ${ }^{134}$ the signal transducer and activator of transcription (STAT) $3^{135}$ and the signal transducer and activator of transcription (STAT) $5{ }^{136}$.

Perhaps from all of these the regulatory role of P53 was the most described in detail by using knock out models of the P53 pathway ${ }^{128}$. In this study a high degree of expressional upregulation of PRL3 under P53 activating genotoxic conditions was revealed. However, in the long term, the upregulation of PRL3 resulted in cell cycle arrest in the G1 phase ${ }^{128}$. Surprisingly, a similar arrest was observable upon the 
knockdown of PRL3 by siRNA in the same cell line, which was shown to be dependent on the presence of a regulator of the P53 pathway termed p19 ${ }^{\text {Arf }}{ }^{128}$. This study suggested that the fine stoichiometry of intracellular PRL3 levels define whether it is acting as a cell cycle promoting factor or initiating growth arrest. This was supported by the fact that PRL3 expression was associated with increased phosphorylation of the AKT kinase only if PRL3 was present in a moderate levels in the cells. This phosphorylation which can be responsible for further downstream cell cycle progression has diminished at the peak of PRL3 expression before the shutdown of cellular growth ${ }^{128}$. However, the authors were not able to replicate the induction of PRL3 by genotoxic stresses in cancer cell lines, suggesting that the phenomena is dependent on the genomic background of a cell ${ }^{128}$.

Other studies have revealed further unsuspected connection between P53 and PRL3, as the knockdown of PRL3 led to the increase of P53 levels. So in these study PRL3 was shown to directly affect the proportion of its own regulator within the cell ${ }^{137}$. The regulation of PRL3 over P53 is observed to work via the activation of the RING finger and $\mathrm{CHY}$ zinc finger domain-containing protein 1 and the mouse double minute 2 protein 137. However, since this observation was made in cancer cells, it is not unequivocal if the results would be the same for non-cancerous cells. Other factors as the TGF $\beta$ was identified as a negative regulator of PRL3 expression as TGF $\beta$ was described to affect the binding of the SMAD2 and SMAD3 transcription factors at the PRL3 promoter ${ }^{120}$. This study proposes a model where the loss of TGF $\beta$ could lead to increased levels of PRL3 expression, which subsequently promotes cancer progression ${ }^{120}$. The study has also shown an increased cell survival upon PRL3 expression by the activation of the $\mathrm{PI} 3 \mathrm{~K} / \mathrm{AKT}$ pathway which is in line with the previously observed AKT activation in the aforementioned studies ${ }^{120}$. Although, the study doesn't reveal the exact mechanism downstream of AKT activation, it shows a dual role for PRL3 and AKT in the resistance against stress-induced apoptosis ${ }^{120}$. Another transcription factor from the $\mathrm{C} 2 \mathrm{H} 2$-type zinc finger family, SNAIL was shown to regulate PRL3 levels by binding to its promoter region ${ }^{138}$. SNAIL is a key transcriptional factor involved in the epithelial-mesenchymal transition process during development ${ }^{139}$. The vascular endothelial growth factor was also connected with PRL3 expression as vascular endothelial growth factor was shown to induce the expression of the myocyte enhancer transcription factor 2 which binds to the promoter of PRL3 enhancing its transcription ${ }^{132}$. Furthermore the signal transducer activator of transcription 3 and 5 (STAT 3 and 5) were also identified to positively regulate the expression of PRL3 in acute myeloid leukemia cells ${ }^{136}$. 
Another study has revealed that the PRL3 mRNA and protein levels do not correlate well with each other in tumor cells, suggesting a regulation over PRL3 at the translational level ${ }^{140}$. By analyzing this issue the study showed an interaction with the 5'-UTR region of PRL-3 mRNA with one of the members of the hnRNP family of RNA- and DNAbinding proteins, the poly (C)-binding protein 1 (PCBP1) ${ }^{140}$. Surprisingly PCBP1 could not inhibit completely PRL3 translation suggesting that this mechanism could serve only as a fine-tuning switch in the regulation of cellular PRL3 levels ${ }^{140}$.

Moreover, PRL3 regulation gets even more complex if one considers the post translational regulations on the protein. PRL3 can be phosphorylated on its Tyr53 by the sarcoma tyrosine-protein kinase (Src) which was shown to play a role in the PRL3 mediated invasion and motility of colon carcinoma ${ }^{141}$. This revealed that the Tyr126 site of PRL3 can also be subjected to phosphorylation, but was not able to identify the responsible kinase ${ }^{141}$. Apart from these, in silico prediction revealed other potential phosphorylation sites in the human PRL3 sequence at Ser13, Thr32, Thr56, Thr64, Thr147 and Thr165 ${ }^{78}$. Thus, the role of different phosphorylation sites on PRL3 still needs to be analyzed.

Furthermore, another regulatory level was described over PRL3 when the FKBP38 protein was identified as a PRL3 interacting protein ${ }^{142}$. FKBP38 was shown to regulate PRL3 by promoting its proteosomal degradation most likely by changing PRL3's conformation by its prolyl cis/trans isomerase activity allowing other proteins to trace it for degradation ${ }^{142}$. The involvement of the ubiquitin mediated degradation processes in the regulation of PRL3 has been further verified by a study detecting polyubiquitinated PRL3 products ${ }^{143}$. Interestingly, this study revealed an interaction between the ubiquitinspecific protease 4 (USP4) with PRL3 which was shown to be responsible for preventing the degradation of PRL3 by decreasing its ubiquitinated products ${ }^{143}$. Also, this study showed a positive correlation between USP4 and PRL3 protein levels in clinical samples of cancerous origin which was not detected in the mRNA level, emphasizing the importance of the translational and posttranslational regulation over PRL3 ${ }^{143}$.

The expression pattern for PRLs is varying in different tissues ${ }^{109}$. Regarding the expression of PRL3 within non-pathological conditions, PRL3 was detected in skeletal muscle and heart tissues ${ }^{144,145}$. PRL3 was also found to be expressed in developing blood vessels and erythrocytes ${ }^{106}$ which is in correspondence with the observed pro-angiogenic effect of PRL3 in cancer cells ${ }^{106}$. Other studies detected PRL3 expression in adult heart, 
intestine, pancreas and other organs ${ }^{123}$ and also in intestinal epithelial cells ${ }^{88}$. In contrast to PRL3`s defined expression in these tissues the other two members of the family show a much greater tissue distribution however, they are surprisingly not overlapping with the tissue types where PRL3 is expressed ${ }^{144}$, suggesting a tissue specific expression for the three PRLs.

\subsubsection{Prenylation and subcellular localization of PRL3}

The PRLs are all special in one unique feature, the presence of a C-terminal CAAX prenylation motif in their sequence, where $\mathrm{C}$ stands for cysteine, $\mathrm{A}$ is an aliphatic amino acid and $\mathrm{X}$ is any amino acid ${ }^{78,146}$. Among the prenyl transferase enzymes that could carry out the transfer of either a farnesyl (C15) or a geranyl (C20) isoprenoid group to the proteins, farnesyltransferase (FT) and geranyl transferase (GGT I) mediate the prenylation of the CAAX sequence ${ }^{146}$, with a distinction that FT prefers methionine, serine or glycine in the $X$ position where GGT I prefers leucine in the X position ${ }^{147}$. Therefore the type of lipid modification is determined by the amino acid at the $\mathrm{X}$ position in the CAAX motif. Prenylation is a common post-translational modification for targeting proteins towards cellular membranes, in order to enable them to act within their relevant signaling network ${ }^{87}$. In the case of PRL3 the CAAX motif is established by the CCVM amino acid sequence ${ }^{88}$ and Cys 170 was shown to be the key amino acid for prenylation 148. The importance of the prenyl group in the membrane attachment of PRL3 was verified by the application of a farnesyl transferase inhibitor, which inhibited the post translational prenylation of PRL3 resulting in the total loss of membrane localization ${ }^{88}$. A stretch of basic amino acids (polybasic domain) in the $\mathrm{N}$-terminal of the protein was also suggested to play a role in the membrane localization of PRL3, based on the sequence similarity to the Ras protein where similar positively charged region is responsible for the fine-tuning of the plasma membrane localization by sequestering it to negatively charged membrane proteins and phospholipids ${ }^{149}$. Therefore the polybasic domain might also play a role determining the specific membrane regions PRL3 is residing.

Immunological localization studies detected PRL3 to reside predominantly at the cytoplasmic site of the plasma membrane and to a lesser extent in endosomal structures. 88. In contrast other members of the family as PRL1 were localized primarily to the nucleus although, a membrane localization was detected for some extent as well 77,88. 
PRL3 membrane localization was further verified by other studies detecting PRL3 in dynamic membrane structures as membrane ruffles and protrusions ${ }^{79}$. After the analysis of PRL3 association with endomembranes, another study suggested an ADP ribosylation factor 1 (ARF1) protein mediated active transport of PRL3 from the endoplasmic reticulum to the plasma membrane. This ARF1 mediated transport was further verified by the loss of membrane attachment of PRL3 upon the addition of Brefedlin A, an inhibitor of ARF1 action ${ }^{88}$. PRL3 was the first from the PRL family the function of which was connected with its localization to the plasma membrane as ectopically expressed PRL3 was detected to associate with the plasma membrane and positively affected the tumorigenic properties of B16F1 mouse melanoma cells ${ }^{148}$. The membrane association was further shown to be critical for PRL3's oncogenic function by the use of a mutant PRL3 protein with a deletion in the C-terminal CAAX prenylation motif ${ }^{148}$.

\subsubsection{PRL3 interacting partners and substrates}

Only a few direct substrates has been verified so far for PRL3. Surprisingly one of these is a lipid, namely the phosphatidylinositol $(4,5)$-bisphosphate $\left(\mathrm{PI}(4,5) \mathrm{P}_{2}\right){ }^{150}$, the others are two membrane associated proteins the integrin $\beta 1$ and ezrin ${ }^{111,151,152}$. The list of predicted PRL3 interacting proteins is a bit longer, it consists of the following proteins: integrin $\alpha 1$, keratin 8, FKBP8, SRC, elongation factor 2, ARF1 ${ }^{129,141,142,153-155}$.

$\mathrm{PI}(4,5) \mathrm{P}_{2}$ was shown to be dephosphorylated under in vitro conditions by PRL3 to $\mathrm{PI}(4) \mathrm{P}$. Our knowledge on the role of PI(4)P in signaling is still scarce, despite that is has been widened significantly in the recent years ${ }^{156}$. PI(4)P was recently shown to be present not only in the trans-Golgi network but to orchestrate signaling at the level of the plasma membrane as well ${ }^{157}$. PRL3 expression was also shown to affect the activation of another molecule ARF1 ${ }^{153}$, which is detected to cooperatively drive the translocation of endosomes to the plasma membrane together with $\mathrm{PI}(4) \mathrm{P}^{158}$. Therefore it is tempting to speculate that the action of PRL3 on PI(4)P production and ARF1 activation is responsible for the induction of plasma membrane targeting of the phosphatase.

Ezrin, a linker of the cytoskeleton and the plasma membrane, was shown to be dephosphorylated at Thr567 in the presence of PRL3 in vitro and suggested as a direct 
substrate for the phosphatase ${ }^{111}$. The dephosphorylation of ezrin in the presence of PRL3 was verified in vivo in a human endothelial cell line however, the study was not able to detect any direct physical interaction between the two proteins ${ }^{111}$. Thus, it is likely that the dephosphorylation of ezrin could be a result of upstream PRL3 action at the plasma membrane. Ezrin was described mainly to be involved in cell adhesion, membrane ruffling, and microvilli formation ${ }^{159}$. The Thr567 site of ezrin was noted to be important for its plasma membrane localization and to the binding of cortical actin to the membrane 160. By applying an unphosphorylatable mutant form of ezrin at Thr567, a central importance was revealed in the establishment of membrane-actin linkages for this site 160. It is important to note that integrin $\beta 1$ has been characterized to bind and phosphorylate ezrin at its Thr567 site ${ }^{161}$. Thus the lack of ezrin phosphorylation could be originating from the upstream, PRL3 caused changes in integrin $\beta 1$.

The association of PRL3 with integrins also shows a complex picture. Both integrin $\alpha$ and $\beta$ has been connected to PRL3 151, 155,162. Integrin $\alpha 1$ was indicated as a possible interacting partner of PRL3 however, no phosphorylation changes were detected on this protein which could be attributed to PRL3 ${ }^{155}$. Integrin $\alpha 2$ expression was shown to be suppressed upon PRL3 expression ${ }^{163}$. PRL3 also affected the subcellular distribution and recycling of integrin $\alpha 5^{153}$. Integrin $\beta 1$ was initially proposed as a PRL3 substrate by the study detecting the interaction between PRL3 and integrin $\alpha 1$, since the $\alpha$ isoforms of integrin does not have any cytoplasmic residues that could act as a substrate for PRL3 ${ }^{155}$ in contrast to the $\beta$ isoforms. The authors of this paper however, could not detect a direct interaction to integrin $\beta 1$ despite the fact that they were able to observe a reduction in the total tyrosine groups on this protein upon PRL3 expression and a higher degree of tyrosine phosphorylation when basal PRL3 expression was abrogated by an siRNA approach in parallel ${ }^{155}$. However, other studies were able to detect a physical interaction between integrin $\beta 1$ and a PRL3, subsequently ${ }^{110,152}$. These studies has connected PRL3 with the dephosphorylation of the Tyr783 site of integrin $\beta 1$ although, it is necessary to note that this was the only phosphorylation site analyzed by this study. The Thr783 site of integrin $\beta 1$ is part of a conserved NPxY motif, necessary for recruiting talin and kindlin, which are responsible for coupling integrin $\beta 1$ to the actin cytoskeleton ${ }^{164}$. It was reported that the viral Src could phosphorylate the tail of integrin $\beta$ on Tyr783 ${ }^{165}$. It was further noted that phosphorylation-dephosphorylation cycles at Tyr783 and Ser785 are required for the movement of integrin $\beta 1$ in and out of focal adhesion contact points ${ }^{166}$. 
It was also reported that the phosphorylation of integrin $\beta 3$ Tyr759, a site located in a very similar position as integrin $\beta$ 1's Tyr783 could block the binding of talin and kindlin 167. Another study analyzed the effect of integrin phosphorylation on tumor formation by using mouse models which were harboring integrins with a tyrosine to phenylalanine substitution on the Tyr783 and/or Tyr795 sites. These mutation has lessened the binding of tail-interacting proteins, as talin and kindlin, resulting in reduced $\beta 1$-mediated focal adhesion kinase (FAK) signaling. The study came to the conclusion that the simultaneous lack of a phosphorylation at Tyr783 and Tyr795 of integrin $\beta 1$ could enhance the occurrence of skin tumors in mice when the animals were treated with a tumor inducing carcinogen 12-O-tetradecanoylphorbol-13-acetate (TPA) ${ }^{168}$. However, it is important to note that the mutation only at the Tyr783 or at the Tyr795 sites did not result in an increase of tumors in the animals ${ }^{168}$.

A recent study analyzed the phosphorylation of the Thr788/789 site of integrin $\beta 1$ in the presence of PRL3 and detected this site to be significantly dephosphorylated ${ }^{151}$. The decrease of phosphorylation on the Thr788/789 site of integrin $\beta 1$ upon PRL3 expression also resulted in impaired integrin clustering at the plasma membrane ${ }^{151}$. The corresponding region of the cytoplasmic tail of integrin $\beta 1$ might be responsible for the regulation of focal adhesion dynamics since the phosphomimetic mutation of Thr788 to phenylalanine enhanced the focal adhesions ${ }^{169}$. On the other hand the mutation of the Tyr788 site to an unphosphorylatable form resulted in the total loss of focal adhesions from the membrane of murine fibroblasts ${ }^{169}$. In a similar study where different phosphodeficient mutants of integrin $\beta 1$ were assessed, the Thr788 mutant showed the greatest decrease in cell attachment even compared to the Tyr783 or to the Tyr795 mutant ${ }^{170,171}$. The Thr788/789 phosphorylation status of integrin $\beta 1$ shows an intriguing fluctuation during cell cycle. Thr788/789 phosphorylation was uniquely present in cells from the G2 phase but not detected in G1 phase cells ${ }^{172}$. Strikingly this phosphorylation was in correlation with the protein's linkage to the actin cytoskeleton and suggested to play a critical switch regulating the progression through the cell cycle ${ }^{172}$. Moreover other studies highlighted the critical role for integrin $\beta 1$ threonine phosphorylation in the anchorage dependent cell cycle regulation mediated by the effector small GTPase Rac1 173 .

Though integrins were among the few detected interaction partners of PRL3 ${ }^{152,155}$, The enzymatic action of PRL3 was only confirmed on integrin $\beta 1^{151,152}$. However, PRL3 
surprisingly affected the total protein amount of integrin $\alpha 2{ }^{163}$ and the subcellular localization of integrin $\alpha 5^{153}$.

According to these observations the integrin receptors are the most probable substrates for PRL3 in the plasma membrane. Therefore in the next part of the chapter, the thesis analyzes the most plausible mechanism through which PRL3 might exert its oncogenic function via the integrin receptors.

1.5.6 The plausible transmission of PRL3 mediated signaling through integrin receptors

The Thr788/789 phosphorylation site of integrin $\beta$ 1was shown to be crucial in integrin mediated cell adhesion ${ }^{169}$ and the interference with the phosphorylation at this site reduced migration and metastasis of cancer cells ${ }^{174}$. The precise regulation of the phosphorylation on the Thr788/789 site is associated with the control of the cell cycle. Integrin $\beta 1$ phosphorylation status on Thr788/789 is regulated by a serine/threonine phosphatase called protein phosphatase 2 (PP2A), which was shown to be crucial for cell cycle progression ${ }^{172}$. PP2A, a phosphatase similar to PRL3, was shown to also regulate integrin $\beta 1$ association with F-actin and with a small GTPase Rac1 ${ }^{173}$. The dephosphorylation of Thr788/789 by PP2A promotes the association of integrin $\beta 1$ and IQGAP1 a guanidine exchange factor responsible for the activation of Rac1 ${ }^{175}$.

Considering that PP2A, enzymatically similar to PLR3, was detected to dephosphorylate integrin $\beta 1$, and is also involved in the regulation of the cell cycle, it is logical to presume that PRL3 is acting through a similar pathway as PP2A. Since PP2A was shown to be associated with the activation of the small GTPase Rac1, the next two chapters are discussing this protein in a greater detail, in order to gain more insight into the mechanism of a possible association between PRL3 and Rac1.

\subsubsection{The role of Rac1 as a possible effector of the PRL3 mediated oncogenesis}

Rac1 or Ras-related C3 botulinum toxin substrate 1 is a small GTPase protein, member of the Rho family of GTPases ${ }^{176}$. The Rho family of GTPase proteins operate under strict controlling mechanisms, which regulates their access to the GTP molecule. Guanine 
nucleotide exchange factors (GEFs) and GTPase activating proteins (GAPs) catalyze their activation via allowing the exchange of GDP to GTP. The inactivation is mediated either by GTP hydrolysis or by the inhibition of GDP/GTP exchange by guanine nucleotide dissociation inhibitors (GDIs) ${ }^{177}$. Rac1 is the most well described member of the Rac subfamily and it was observed to play a central role in many cellular processes such as cell cycle regulation ${ }^{178}$, cellular adhesion ${ }^{179}$, differentiation ${ }^{180}$, cell migration and actin polymerization ${ }^{181}$. Rac1 was shown to be important in various oncogenic pathways including tumor initiation and progression, invasion and metastasis ${ }^{182}$. Rac1 undergoes a dual posttranslational modifications, prenylation and reversible palmitoylation in its C-terminal CAAX motif. These posttranslational modifications mediate its association with the plasma membrane which is necessary for Rac1 to exert its function ${ }^{183}$. Like all the members of the Rho GTPase family, Rac1 also acts like a molecular switch by cycling between an inactive GDP bound and an active GTP bound state. The GTP binding is initiated and fine-tuned by GEFs at the plasma membrane. Typically GEFs are induced by activated receptor dependent kinases and when the signal falls off, the constantly present GAPs remove the $\gamma$-phosphate of the GTP and return the active GTPase to a GDP bound inactive form ${ }^{184}$. Tiam1, Trio, and GEF-H1 are GEF proteins that are responsible for catalysis of the GDP-GTP exchange reaction specifically for Rac1 ${ }^{180}$. The diversity of posttranslational lipid modifications on Rho GTPases provide a functional diversity for these molecules by giving preferential association with distinct subcellular compartments. Rac1 is modified by geranyl-geranylation via a thioester group, catalyzed by a geranyl-geranyl transferase at its CAAX motif. Palmitoylation also occurs upstream of this motif at a cysteine residue via a thioester bond. Furthermore the membrane localization of Rac1 is also modulated by the presence of a positively charged basic amino acid stretch of 6 amino acids at the N-terminal of the protein, which could interact with negatively charged membrane phospholipids or membrane proteins ${ }^{183}$. Palmitoylation of Rac1 requires its prior prenylation and the intact C-terminal polybasic region. Non-palmitoylated Rac1 shows lower association with raft membranes and a decreased GTP loading. Therefore, palmitoylation is considered to be the key signal which guides Rac1 to lipid rafts within plasma membrane 185.

The localization of Rac1 to raft domains could lead to the activation of many signaling events. Furthermore the localization of Rac1 into specific microdomains such as the caveolae could regulate the activation state of the molecule ${ }^{186}$. Moreover caveolae could serve as a regulatory platform over Rac1 by orchestrating its dislocation from the 
membrane to the endocytic system, thereby not allowing Rac1 to interact with its GEFs, downstream effectors or the actin cytoskeleton ${ }^{31,32}$. This happens characteristically during the loss of integrin mediated attachment signals.

Rac1 was also suggested to participate in the cellular response to various kinds of stresses. Stress condition such as heat stress can induce the translocation of Rac1 to the lipid raft fraction of the plasma membrane which can subsequently lead to its activation and hypothesized to participate in the lateral domain rearrangements of the cholesterol rich plasma membrane domains. These membrane platform alterations are described to initiate signaling which could mitigate stress responsive protein expression in the form of heat shock proteins. This data clearly shows an excellent example where Rac1 dynamic interaction with the plasma membrane could affect downstream gene expression upon stress conditions ${ }^{187}$.

\subsubsection{Rac1 control over cell proliferation}

In parallel with the studies which detected Rac1 to partition into the caveolae region of the plasma membrane ${ }^{188}$ it was also suggested that these membrane domains are essential for Rac1 to transmit the extracellular adhesion forces towards the cell interior ${ }^{31}$. As it was described before, in the absence of integrin mediated contacts cells inactivate Rac1 and its associated signaling by internalization of the caveolae components into recycling endosomes ${ }^{32}$ which results in the temporal reduction of ordered domains in the plasma membrane ${ }^{189}$. Thus, the detachment of a cell from its substrate leads to the loss of integrin and subsequently Rac1 activity which results in a cell cycle arrest. The deregulation of this cellular growth control mechanism results in the subsequent development of anchorage-independent growth, which could contribute to cancer progression ${ }^{73,190}$. The progression through the cell cycle is orchestrated and managed by a number of cyclin-dependent kinases. From all of these, cyclin D1, a key factor in determining G1-phase progression, is affected by the adhesion forces influencing the cell. The amount of cyclin D1 in the nucleus is highly sensitive to adhesion forces and the length of the G1 phase is dependent on intracellular tension and the formation of actin stress fibers ${ }^{191}$. The level of cellular cyclin D1 is regulated on a multi-scale level within the cell, among these Ras and Rho GTPases act in parallel as upstream orchestrators ${ }^{192}$. Among the GTP binding molecules, the regulatory role of Rac1 over cyclin D1 was 
shown to be interconnected with integrins and integrin activation ${ }^{193}$. Several evidence strengthens this connection as cyclin D1 synthesis was prevented by the introduction of a dominant negative Rac1 in adherent conditions and was rescued in the presence of a dominant active Rac1, even in the absence of an extracellular matrix which would support integrin activation ${ }^{193}$. This was further verified by the inhibition of Rac1 activity via the Rac1 inhibitor NSC23766, where the inhibition of Rac1 was shown to induce a cell cycle arrest through downregulation of cyclin D1 ${ }^{194,195}$. Others also highlighted that the activation of the p21-activated serine/threonine kinase (PAK) 1, a key Rac1 effector, could also play a role in this process ${ }^{32}$. The importance of the caveolae domains in Rac1 mediated cyclin D1 regulation were further validated by the use of mouse embryonic fibroblast cell lines lacking caveolin 1 expression ${ }^{73}$. Studies concentrating on the pathway that could contribute to the effect of Rac1 on cyclin D1 levels described a key role for the AKT kinase and for the glycogen synthase kinase $3 \beta$ (GSK3 $\beta$ ) in the fine regulation over cyclin D1 ${ }^{191,196}$. The phosphorylation of cyclin D1 at Thr286 residue by GSK3 $\beta$ regulates cyclin D1 level since this phosphorylation directly results in the shuttle of cyclin D1 out from the nucleus to the cytoplasm and its subsequent ubiquitination and proteasome mediated degradation ${ }^{197}$. The AKT kinase acts as an upper regulator in this process by phosphorylating GSK3 $\beta$ at its inhibitory site at serine 9 resulting in increased cyclin D1 stability ${ }^{198}$. However, AKT kinase needs to be phosphorylated at its catalytic site at Ser473 by PAK1 to affect GSK3 $\beta$ phosphorylation to ${ }^{199,200}$. As described earlier PAK1's function is greatly intertwined with Rac1's activation. The membrane localized Rac1 initiates F-actin polymerization via interacting with the heterodimer of the Arp2/3 proteins. The modified cytoskeleton by Rac1 can act as a membrane organizer ${ }^{180}$. Subsequently actin polymerization recruits the coronin1A/ArhGEF7 complex which associates with the PAK 1, which serves as a downstream effector of Rac1 action. This protein complex further interacts with GDI bound Rac1-GDP molecules initiating their membrane localization and exerting a positive feedback loop ${ }^{201,202}$. In summary, the adhesion mediated signaling axis that regulates cyclin D1 could be drawn as follows: Rac1-GTP/PAK1/AKT/GSK3ß/cyclin D1.

The above detailed cell growth regulatory function and caveolae localization of Rac1 is further emphasizing a possible connection between Rac1 and PRL3. Also, PRL3 was observed to affect leukemia progression through elevated cyclin D1 levels ${ }^{122}$. As PRL3 was associated with cell cycle regulation in non-cancerous MEF cells it seems logical to assume that it can act as a cell growth regulatory factor during the cell cycle by the 
regulation of integrin-Rac1 signaling at the plasma membrane. The over activation of this pro-growth signaling could explain PRL3`s role in the establishment of primary tumors and the observed therapy resistant phenotype of PRL3 expressing cancers. In summary, the analysis of the available literature data draws a picture where PRL3 modulates cell growth by the dephosphorylation of integrin $\beta 1$ which further affects the activation of downstream Rac1 mediated cell growth signaling. 


\section{Aims of the Thesis}

Our concept of the plasma membrane has evolved from a uniform lipid bilayer with randomly floating proteins, to a highly complex cellular structure that plays a central role in the transmission of environmental signals and in the regulation of cellular events. As our technology advances at one hand it allows us to have a deeper insight into the organization of membranes but on the other hand it leaves us with a more troubled state of mind by revealing the complexity still ahead of us. However, the statement that membranes are unavoidable in the understanding of either physiological or pathological cellular behavior stays solid over time. By keeping this philosophy in mind, the main goal of the thesis was to characterize physiologically and pathologically relevant events at the level of the plasma membrane and couple these to the regulatory role of detergent resistant plasma membrane microdomains. Thus, the aims of the present work were:

- to determine how a cell can maintain its membrane integrity under challenging conditions by analyzing the planar distribution of membrane microdomains during stress and by analyzing the effect of membrane fluidity induced HSP expression on membrane order.

- to decipher a possible cellular mechanism for maintaining the integrity of the plasma membrane

- to investigate the membrane interaction and sub-membrane localization and possible lipid raft association of a known membrane bound oncogene, PRL3

- to define the oncogenic signaling mechanism behind PRL3 action

- to further characterize the stress association of PRL3

- to connect the action of PRL3 to its pro-tumorigenic action in vivo 


\section{Materials and methods}

3.1 Cell culture and treatments

B16F0 (ATCC, CRL-6322), B16F1 (ATCC, CRL-6323) and B16F10 (ATCC, CRL6475) mouse melanoma cells were maintained in Roswell Park Memorial Institute medium (Thermo Fischer Scientific), media were supplemented with $10 \%$ fetal bovine serum (Thermo Fischer Scientific), $100 \mathrm{U} / \mathrm{ml}$ penicillin and $100 \mu \mathrm{g} / \mathrm{ml}$ streptomycin (Sigma Aldrich). The cells were incubated in a humidified atmosphere containing 5\% $\mathrm{CO}_{2}$ at $37^{\circ} \mathrm{C}$. Cells were treated at the noted concentrations of doxorubicin, cisplatin, etoposide and paclitaxel (all from Sigma Aldrich). Etoposide and paclitaxel were solubilized in DMSO. NSC23766 trihydrochloride (Sigma Aldrich, SML0952) was used to inhibit Rac1 function at the indicated concentration. In every experimental conditions except stated otherwise the cells were treated with the chemicals for 24 hours. All cell lines were routinely tested for mycoplasma contamination.

3.2 Transfection, clone selection and stable expression of PRL3 in B16F0 cells Human PRL3 cDNA clone was purchased from Origene in a pCMV6-XL5 expression vector. The PRL3 sequence were cloned into the EcoRI/XhoI sites of pcDNA 4/TO (Thermo Fischer Scientific). For inducible protein expression pcDNA6/TR (Thermo Fischer Scientific) was used as a source of tetracycline repressor. B16F0 cells were transfected with pcDNA 4/TO or pcDNA4/TO-hPRL3 to achieve constitutive expression. In order to create clones with inducible protein expression pcDNA6/TR and pcDNA4/TO-hPRL3 were co-transfected to B16F0 cells in an equal molar with ExGen 500 (Thermo Fischer Scientific) according to manufacturer's instructions. 24 hours after the transfection cells were seeded into 96-well plates and resistant colonies were selected by the simultaneous addition of Zeocin at $700 \mu \mathrm{g} / \mathrm{ml}$ and Blasticidine at $8 \mu \mathrm{g} / \mathrm{ml}$ (both from Thermo Fischer Scientific) or Zeocin only. Cells were allowed to grow for a period of 12 days then the isolation of individual colonies was carried out. The expression of PRL3 was induced by the addition of doxycycline hyclate (Sigma Aldrich, D9891) to the cell culture media for 24 hours at the concentration of $2 \mu \mathrm{g} / \mathrm{ml}$. The HSP25 expressing cell lines were already established in our laboratory. Cell propagation and protein 
induction in these lines were carried out in the same fashion as with the PRL3 expressing cells.

\subsection{Western blot analysis}

Cells were lysed in 2X Laemmli buffer $(130 \mathrm{mM}$ TrisHCl pH 6.8, $10 \% \beta$ mercaptoethanol, $4 \%$ SDS, $20 \%$ glycerin, $0.01 \%$ bromophenol blue) and equal amounts of proteins were run on SDS/PAGE followed by the transfer to a PVDF membrane. Membranes were probed with anti-PRL3 (Santa Cruz Biotechnology, 1:500, sc-130355), anti-sodium/potassium ATPase (Santa Cruz Biotechnology, 1:500, sc-48345), anti-cyclin D1 (Santa Cruz Biotechnology, 1:500, sc-8396), anti-Caveolin 1 (Thermo Fischer Scientific, 1:1000, 7C8), anti-Integrin $\beta 1$ (Abcam, 1:2000, ab183666 ), anti-Integrin $\beta 1$ phospho T788 + T789 (Abcam, 1:500, ab5189), anti- GSK-3 $\alpha / \beta$ (Cell Signaling Technology, 1:1000, 5676) anti-phospho GSK3 $\beta$ Ser9 (R\&D Systems Inc., 1:1000, AF1590) , anti-Rac1 (EMD Millipore, 1:1000, 05-389), anti-AKT (Cell Signaling Technology, 1:1000, 9272), anti-AKT phospho Ser473 (Cell Signaling Technology, 1;2000, 9271), anti-HSP25 (ENZO, 1:1000, ADI-SPA-801), anti-HSP70 (ENZO, 1:2000, ADI-SPA-810), and anti-GAPDH (Sigma Aldrich, 1:20000, G9545) antibodies. Secondary rabbit or mouse antibodies (Sigma Aldrich) were used in 1:50000 dilutions for 1 hour at room temperature. Data are representative of three independent experiments.

\subsection{Immunoprecipitation}

Cells cultured in $10 \mathrm{~cm}$ dishes were washed with PBS and lysed by the addition of $300 \mu \mathrm{l}$ of ice-cold RIPA buffer (10 mM Tris-Cl, 1 mM EDTA, $0.5 \mathrm{mM}$ EGTA, $1 \%$ Triton X$100,0.1 \%$ sodium deoxycholate, $0.1 \% \mathrm{SDS}, 140 \mathrm{mM} \mathrm{NaCl}, \mathrm{pH} 7.5$ ) supplemented with the appropriate dilution of a phosphatase (Sigma Aldrich, P5726) and protease inhibitor (Sigma Aldrich, P2714) cocktail. Insoluble material was removed by centrifugation at $12,000 \mathrm{~g}$ for 5 minutes. Before the addition of antibody protein concentration was adjusted to $1 \mathrm{mg} / \mathrm{ml}$ and $500 \mu \mathrm{g}$ of total protein was used for each reaction. The lysates were precleared with Protein G Sepharose beads (GE healthcare Life Sciences, 17061801) for 30 minutes before the immunoprecipitation reaction. For the immunoprecipitation an anti-AKT (Cell Signaling Technology, 1:50, 9272) antibody was mixed with the indicated sample lysates and the mixtures were incubated for 16 hours at $4^{\circ} \mathrm{C}$. Then the immunocomplexes were incubated with Protein G Sepharose beads for 2 
hours at $4^{\circ} \mathrm{C}$ then washed three times with ice-cold PBS. The beads were suspended in 40 $\mu \mathrm{l}$ of $2 \mathrm{X}$ Laemmli buffer and the immunoprecipitates were analyzed by western blotting. The co-immunoprecipitations were carried out with the same conditions except the cells were lysed in a low detergent buffer $(10 \mathrm{mM}$ Tris-Cl, $1 \mathrm{mM}$ EDTA, $0.5 \mathrm{mM}$ EGTA, $0.5 \%$ Triton X-100, $0.1 \%$ sodium deoxycholate, $140 \mathrm{mM} \mathrm{NaCl}, \mathrm{pH} 7.5$ ) and anti-Rac1 (EMD Millipore, 1:50, 05-389) was used for the reaction. In the case of overlapping antibody combinations, samples were analyzed in separate membranes.

\subsection{Rac1-GTP pull-down assay}

Rac1-GTP detection was carried out with the commercial EZ-Detect Rac1 Activation Kit (Pierce, 89856Y) according to the manufacturer's instructions. Briefly, cells were lysed in the provided lysis buffer and the solution was cleared by centrifuging with $16,000 \mathrm{~g}$ at $4^{\circ} \mathrm{C}$ for 15 minutes. After the measurement of protein concentration $100 \mu \mathrm{g}$ of protein was separated from each sample and mixed with $2 \times$ Laemmli buffer as a control for total Rac1 expression. For the pulldown experiments an equal amount of $500 \mu \mathrm{g}$ of protein was transferred from the supernatant to glutathione beads containing $20 \mu \mathrm{g}$ of GST-Pak1PBD. The mixtures were incubated with gentle rocking for 1 hour at $4^{\circ} \mathrm{C}$, then centrifuged with $6000 \mathrm{~g}$ for 30 second and washed three times with ice cold PBS. The beads were suspended in $50 \mu 1$ of $2 X$ Laemmli buffer. The GTP-bound fraction of Rac1 and the total Rac1 fraction were analyzed with western blotting.

\subsection{Soft agar assay}

$1 \times 10^{4}$ of B16F0, B16F0-pcDNA4/TO (mock) or B16F0-pcDNA4/TO-hPRL3 (PRL3) cells were seeded into a well of a 24 well plate in RPMI media containing $0.7 \%$ agarose (Sigma Aldrich, A3768) and propagated for 14 days. Media has been changed in every three days. After 14 day incubation period the viable cell number was measured by the resazurin dye based alamar Blue assay (Thermo Fischer Scientific, DAL1025) according to the manufacturer's instructions. The spheroid colonies were incubated with the dye containing media for 5 hours. Fluorescence was measured with a microplate fluorimeter (Thermo Fischer Scientific, Fluoroskan Ascent FL 5210450) adjusting the excitation filter to $560 \mathrm{~nm}$ and the emission filter to $590 \mathrm{~nm}$. The detected fluorescence is in a direct correlation with the number of living cells. 


\subsection{Tumor growth assay}

C57BL/6J mice were obtained from the colony of the National Institute of Oncology, Budapest. Animal welfare and experimental procedure were performed in accordance with related regulations of the ARRIVE guidelines ${ }^{203}$ and with the animal welfare regulations of the host institute (permission number: 22.1/722/3/2010). For the tumor growth assay subconfluent B16F0, B16F0-pcDNA4/TO (mock) or B16F0-pcDNA4/TOhPRL3 (PRL3) cells were harvested and washed three times with PBS. $6 \times 10^{5}$ cells were inoculated subcutaneously in the volume of $0.1 \mathrm{ml}$ PBS into the dorsal flanks of 8-week old syngenic C57BL/6J mice as described previously ${ }^{204}$. The subcutaneous tumors were allowed to propagate for the indicated days. Tumor size was measured three times a week with a caliper and expressed in $\mathrm{mm}^{3}$ with the formula for the volume of a prolate ellipsoid (length $\mathrm{x}$ width $2 \mathrm{x} \pi / 6$ ) as described previously ${ }^{205}$.

\subsection{Plasma membrane isolation}

This isolation method was carried out as described previously in Maeda et al. ${ }^{206}$. Briefly, $3 \times 10 \mathrm{~cm}$ subconfluent plates from the indicated cells were washed three times with ice cold PBS, scrapped and collected in $500 \mu \mathrm{l}$ ice cold TNM buffer $(10 \mathrm{mM} \mathrm{NaCl}, 1.5 \mathrm{mM}$ $\mathrm{MgCl} 2,10 \mathrm{mM}$ Tris-HCl, $\mathrm{pH}$ 7.4), supplemented with the appropriate dilution of phosphatase (Sigma Aldrich, P5726) and protease inhibitor (Sigma Aldrich, P2714) cocktail. Then cells were homogenized with the addition of glass beads ( $\leq 106 \mu \mathrm{m}$, Sigma Aldrich, G4649) to the lysate followed by a thorough vortexing. Lysates were spun down at $2000 \mathrm{~g}$ at $4{ }^{\circ} \mathrm{C}$ for $5 \mathrm{~min}$. Supernatant was collected and centrifuged at $8000 \mathrm{~g}$ at $4{ }^{\circ} \mathrm{C}$ for 15 min in F28/13 rotor in a Sorvall (RC-28S) super-speed centrifuge. Supernatant was discarded and pellet was re-suspended gently in $8 \mathrm{~mL}$ of TNM buffer. Parallel to this $35 \%$ sucrose in TNM buffer was prepared and $4 \mathrm{ml}$ filled in ultra-centrifuge tubes (Beckman Coulter, 344059) on top of which re-suspended pellet was delicately layered. The tubes were centrifuged at $100,000 \mathrm{~g}, 4{ }^{\circ} \mathrm{C}$ for $3 \mathrm{~h}$ in SW41 rotor in a Sorvall ultracentrifuge (WX Ultra80). Interphase of each sample was collected into TNM buffer and centrifuged again at $100,000 \mathrm{~g}, 4^{\circ} \mathrm{C}$ for $1 \mathrm{~h}$ in SW41 rotor. Supernatant was removed and the pellet (PM fraction) was dissolved in 2X Laemmli Buffer. The protein content of the fractions were analyzed by western blotting. 
3.9 Detergent-free purification of caveolin enriched membrane fractions

This isolation method was carried out as described previously in Song et al. ${ }^{207}$. Briefly, 3 x $10 \mathrm{~cm}$ sub-confluent plates of B16F0-pcDNA4/TO-hPRL3 (PRL3) cells were washed twice with ice-cold PBS and were scraped into $1 \mathrm{ml}$ of sodium carbonate buffer $(500 \mathrm{mM}$ sodium carbonate, $25 \mathrm{mM}$ 4-morpholineethane sulfonic acid, $150 \mathrm{mM} \mathrm{NaCl}, \mathrm{pH}$ 11) supplemented with the appropriate dilution of phosphatase (Sigma Aldrich, P5726) and protease inhibitor (Sigma Aldrich, P2714) cocktail. Then cells were homogenized with the addition of glass beads $(\leq 106 \mu \mathrm{m}$, Sigma Aldrich, G4649) with thorough vortexing. Followed by a centrifugation at $2000 \mathrm{~g}$ for 10 minutes to pellet the nuclear fraction and the beads. The supernatant of the homogenate was then adjusted to $45 \%$ sucrose by the addition of $0.56 \mathrm{ml}$ of $80 \%$ sucrose in sodium carbonate buffer and placed at the bottom of an ultracentrifuge tube (Beckman Coulter, 344059). A 5-35\% discontinuous sucrose gradient was formed above the $45 \%$ layer by adding $6.25 \mathrm{ml}$ of $35 \%$ sucrose and $4.2 \mathrm{ml}$ of 5\% sucrose, both in sodium carbonate buffer. The gradient was centrifuged at 170,000 $\mathrm{g}$ for 24 hours using a Beckman SW41 rotor in a Sorvall ultracentrifuge (WX Ultra80). For the analysis of the resulting gradient $1 \mathrm{ml}$ fractions were collected from the top to the bottom of the gradient. The fractions were further analyzed by western blotting.

\subsection{Confocal microscopy}

The indicated cells were washed with PBS and fixed with 4\% paraformaldehyde (Sigma Aldrich) for $8 \mathrm{~min}$ at room temperature then permeabilized and blocked with PBS containing $0.1 \%$ Saponin and 1\% bovine serum albumin (Sigma Aldrich) for 60 minutes at room temperature followed by an incubation with anti-Rac1 (EMD Millipore, 1:100, 05-389), anti-PRL3 (Santa Cruz Biotechnology, 1:50, sc-130355) or with anti-cyclin D1 antibody (Santa Cruz Biotechnology, 1:100, sc-8396) for 16 hours at $4^{\circ} \mathrm{C}$. After washings cells were incubated with anti-mouse IgG conjugated with Alexa488 (Thermo Fischer Scientific, 1:300, A21202) or Alexa555 (Thermo Fischer Scientific, 1:300, A31570) for 30 minutes at room temperature. Images were taken with a TCS SP5 laser scanning inverted confocal microscope with Leica LAS software (Leica) using a 63x oilimmersion. The nuclei were counterstained with $1 \mu \mathrm{g} / \mathrm{ml}$ 4,6-diamidino-2-phenylindole (Thermo Fischer Scientific, D1306). 
3.11 Imaging of di-4-ANNEPDHQ and calculation of General Polarization (GP)

The indicated cells were labelled with the membrane incorporating dye di-4ANNEPDHQ (Thermo Fischer Scientific, D36802) added to the growth media at the final concentration of $5 \mu \mathrm{M}$ and incubated for 5 minutes at a humidified atmosphere containing 5\% $\mathrm{CO} 2$ at $37^{\circ} \mathrm{C}$. Image acquisition was carried on a TCS SP5 laser scanning inverted confocal microscope with Leica LAS software (Leica) using a 63x oilimmersion objective. Argon ion laser at $488 \mathrm{~nm}$ was used for excitation and detection ranges of PMTs were set to $500-580 \mathrm{~nm}$ and $620-720 \mathrm{~nm}$, respectively for the two emission channels. di-4-ANEPPDHQ data are typically displayed as pseudocolored GP images. The GP values are calculated according to the following equations:

$\mathrm{GP}=\left(I \_(500-580)-\mathrm{G} I \_(620-750)\right) /\left(I \_(500-580)+\mathrm{G} I \_(620-750)\right)$.

Where I represents the intensity in each pixel in the indicated spectral channel (numbers are in $\mathrm{nm}$ ) and $\mathrm{G}$ represents the calibration factor which is to compensate the differences in the efficiency of collection in the two channels.

3.12 Preparation of plasma membrane sheets

B16F10 cells were grown on fibronectin $(25 \mu \mathrm{g} / \mathrm{ml}$, Sigma Aldrich, F1141) treated coverslips overnight. Cells were rinsed with ice-cold PBS and with cold hypotonic buffer (23mM KCl, 10mM HEPES pH 7.5, 2mM MgCl2, 1mM EGTA) three times. Cells are then placed into sonication buffer $(70 \mathrm{mM} \mathrm{KCl}, 30 \mathrm{mM}$ HEPES $\mathrm{pH} 7.5,5 \mathrm{mM} \mathrm{MgCl}$, 3mM EGTA) containing beaker. Sonication was carried out with $19 \mathrm{~mm}$-tip fitted Branson sonifier cell disruptor B-30 with optimized operation settings (power 1.5, duty cycle $80 \%$ ) for 3 times. Finally sonicated coverslip was rinsed with sonication buffer to be further used for the microscopy experiments. The membranes were treated with $1 \mu \mathrm{M}$ recombinant HSP25 (Abcam, ab92457) in a 1\% BSA solution.

\subsection{Image based Fluorescent Correlation Spectroscopy (ImFCS)}

Cells were seeded into glass bottom dishes (MatTek Corporation,MA, USA) 24 hours before the experiment. Measurement was performed in culturing media without phenol red at $37^{\circ} \mathrm{C}$ with $\mathrm{CO}_{2}$ administration. A POC-R cell cultivation system (Zeiss, Jena, Germany) was attached to the stage of microscope for obtaining the growth conditions. Objective type Total Internal Reflection illumination was used for achieving the thinnest 
excited sample volume with a high numerical aperture objective (alpha Plan-FLUAR 100, Zeiss). Excitation wavelength 488 nm from a Spectra-Physics Stabile 2018 (SpectraPhysics, CA, USA) laser as light source was introduced to the microscope (Zeiss Axiovert 200) by two tilting mirrors. The laser beam was focused on the back focal plane of the objective after a $488 \mathrm{~nm}$ cleanup filter and aZT488/647/780rpc-UF1 dichroic mirror (Chroma Technology GmbH, Olching, Germany). Sample signal was collected by the objective and filtered by a 535/70 emission filter (Chroma). For acquisition we used a ProEM512 EMCCD camera (Prinston Instruments, NJ, USA) with $3 \mathrm{~ms}$ effective exposure time and 20x40 pixel acquisition area per measurement (pixel size $0.16 \mu \mathrm{m}$ ) ImFCS plugin (http://www.dbs.nus.edu.sg/lab/BFL/imfcs_image_j_plugin.html) for ImageJ software was used for data evaluation. The data analysis was carried out as the following. The autocorrelation functions (ACFs) for every pixels were calculated using a multi-tau correlation scheme ${ }^{208}$. An exponential of polynomial bleach correction was used to correct data before fitting. For obtaining the diffusion coefficient (D) for all pixels ACFs were fitted according to the equation below.

$$
G(\tau)=\frac{1}{N}+\left[\frac{\operatorname{erf}(p(\tau))+\frac{\left(e^{-(p(\tau))^{2}}-1\right)}{\sqrt{\pi p(} \tau)}}{\operatorname{erf}(p(0))+\frac{\left(e^{-(p(0))^{2}}-1\right)}{\sqrt{\pi p(}(0)}}\right]^{2}+G_{\infty} p(\tau)=\frac{a}{\sqrt{4 D \tau+\omega_{0}^{2}}}
$$

Where $G(\tau)$ is the ACF in dependence of the correlation time $(\tau), N$ is the number of detected particles, $\mathrm{a}$ is the pixel size and $\omega_{0}$ is the $1 / \mathrm{e}^{2}$ radius of the Gaussian approximation of the point spread function. To identify and describe the mode of membrane organization by investigating the size-dependency of diffusion coefficient we used the Imaging FCS type of FCS diffusion law ${ }^{209}$. According to that the diffusion time $\left(\tau_{D}\right)$ of the fluorescent probe depends on the observation area $\left(A_{e f f}\right)$ as it is described by

$$
\tau_{D}\left(A_{e f f}\right)=\tau_{0}+\frac{A_{e f f}}{D}
$$

where $A_{e f f}$ is the area of the membrane where the particle travels which is calculated by the convolution of the detection area with the point spread function. $\tau_{0}$ is the intercept of the diffusion law plot on the y axis of $A_{e f f} / \mathrm{D}$ against $A_{e f f}$. This provides information on confinement. Diffusion law is plotted by using different $A_{\text {eff }}$ values which were calculated by post- inquisitional binning of pixels. In the case of free diffusion $D$ is 
constant regardless of $A_{e f f}$ so it shows a straight line passing through the origin ( $\pm 0.1 \mathrm{~s}$ ) in the diffusion law graph. A heterogeneous system with domains however, allows spatial scale dependent diffusion which results remarkable different diffusion law plot with positive or negative intercepts for domain partitioning diffusion, respectively ${ }^{209}$.

\subsection{Domain size analysis of sphingomyelin rich microdomains}

B16F10 cells were incubated with $0.2 \mu \mathrm{M}$ BODIPY FL C5 sphingomyelin for $20 \mathrm{~min}$. After staining, the cells were imaged with an Olympus (Melville, NY) Fluoview 1000 microscope by using the $488-\mathrm{nm}$ argon-ion laser line for the excitation. Pictures were taken with a resolution of $1,600 \times 1,600$; the final magnification was $\times 300$. The domain size was analyzed with the freeware ImageJ software (www.uhnresearch.ca/facilities/ wcif/imagej), with its Fourier transform (FFT) filter. The domains were sorted according to diameter. Diameters were calculated as $2 \mathrm{X}(\mathrm{N}$ pix $/ \pi)-2$, where $\mathrm{X}$ is the size of a pixel in nanometers, and $\mathrm{N}$ pix is the number of pixels covering the actual domain.

\subsection{Electron microscopy}

The indicated cells were washed with PBS and fixed in $5 \mathrm{ml}$ of $0.1 \mathrm{M}$ cacodylate buffer containing 1\% glutaraldehyde (Sigma Aldrich, G5882) and 1\% osmium tetroxide (Sigma Aldrich, 75632) for 1 hour at room temperature followed by a wash with cacodylate buffer. Subsequently, the cells were scraped in $500 \mu 1$ cacodylate buffer and centrifuged with $3000 \mathrm{~g}$ at $4^{\circ} \mathrm{C}$. After the removal of the supernatant the fixed cells were washed with $0.05 \mathrm{M}$ glycin in PBS, and collected in PBS containing $1 \%$ gelatin and pelleted in $12 \%$ gelatin in PBS. The cell pellet was solidified on ice and cut into small blocks. The samples were dehydrated with ethanol and stained with $1 \%$ uranyl acetate (in $70 \%$ ethanol for $1 \mathrm{~h}$, room temperature) prior to araldite embedding. Ultrathin sections were contrast-stained with uranyl acetate and lead citrate. The samples were analyzed with a Hitachi H-7600 (Tokyo, Japan) transmission electron microscope. 


\section{Results}

4.1 Membrane microdomains in cellular signaling: the role of membrane fluidity and lateral domain mobility in cellular stress sensing:

Heat and benzyl alcohol fluidizes the membrane in a similar manner

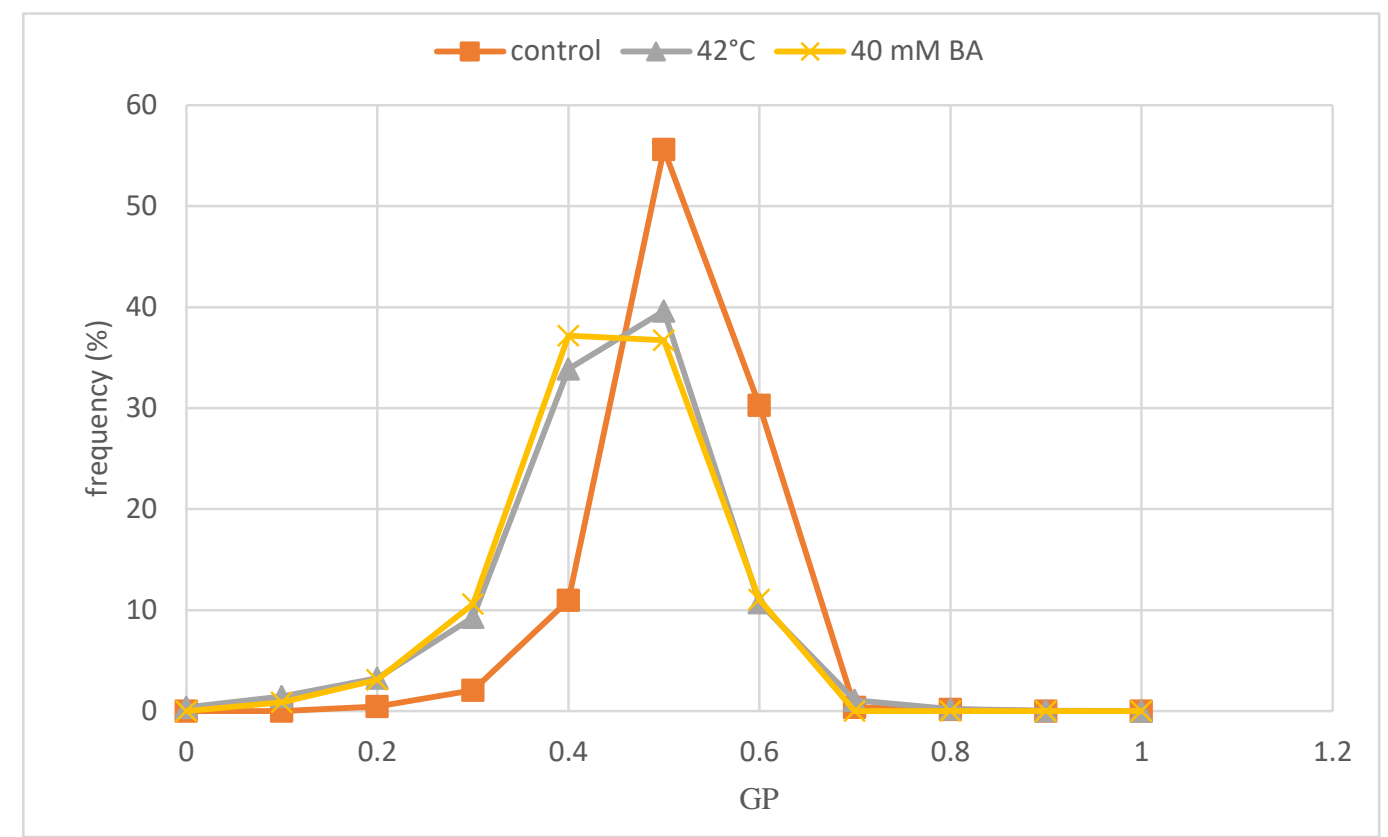

Figure 1. GP value changes at $42^{\circ} \mathrm{C}$ or upon BA treatment. B16F10 cells were treated with heat $\left(42^{\circ} \mathrm{C}\right)$ or with the non-proteotoxic membrane fluidizer benzyl-alcohol $(40 \mathrm{mM})$. for 1 hour.

Membrane order was assessed by the addition of the membrane incorporating dye di-4ANNEPDHQ. Data is expressed as GP distribution of at least 80 PM segments, from three independent experiments. Data is shown as a frequency distribution.

In our experiments we studied the effect of membrane fluidization, a stimulus associated with membrane reorganization and stress signaling. The fluidizing effect of heat on the plasma membrane is a well characterized phenomena ${ }^{53,210}$. However, for decades the importance of this fluidization in stress sensing was left unrecognized as the initial models of the heat shock response concentrated solely on the protein denaturing effect of heat. In this study we sought to unravel the effect of membrane fluidization on downstream stress signaling, heat shock protein expression and cellular stress adaptation. In order to model the effects of heat induced membrane fluidization we chose to use a 
known membrane fluidizing agent, benzyl alcohol (BA). BA was shown by previous studies to induce membrane fluidization similar to heat without inducing protein denaturation ${ }^{53}$. Thus the introduction of BA does not affect stress signaling pathways connected with protein denaturation. To assess the changes in the physical state of the cell membrane we used an environmentally-sensitive fluorescence probe di-4ANEPPDHQ that inserts into the membrane bilayer, distributes equally between lipid phases and exhibits an emission spectral shift depending on the lipid phase state. The ratio of the intensities of the two emission spectra of the probe gives the value of the generalized polarization (GP) which provides a measure of membrane order, in the range between -1 (liquid-crystalline) and +1 (gel). We treated B16F10 cells with heat and the artificial membrane fluidizer BA. The cells were subsequently incubated with the di-4ANEPPDHQ dye in order to analyze the changes in membrane order upon the treatments. Both heat and BA caused a distinct membrane fluidization as the membrane structure became more disordered compared to non-treated controls (Fig. 1.). Thus with the BA we were able to model the fluidizing effect of heat on the membrane structure.

4.2 Membrane fluidization increases the coalescence of sphingomyelin rich membrane domains on the cell surface

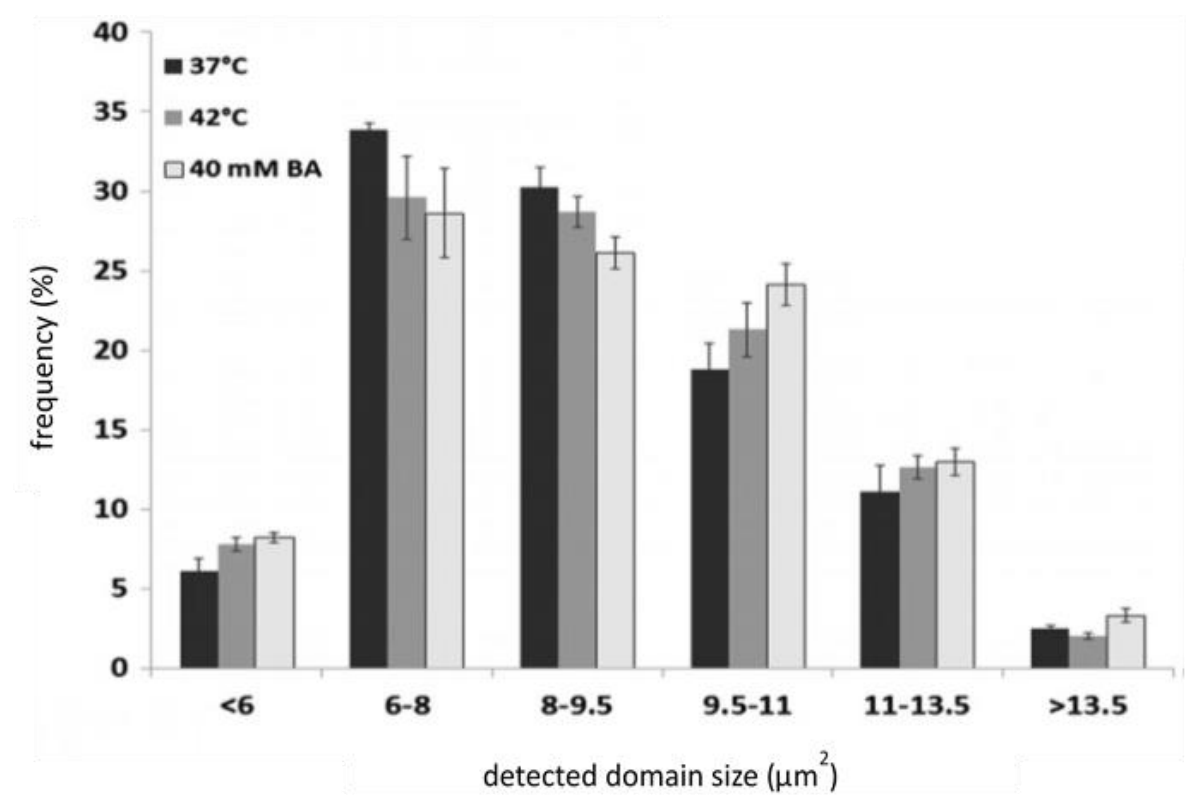

Figure 2. Redistribution of the sphingomyelin membrane domains on the surface of B16F10 cells, monitored by a BODIPY FL C5 sphingomyelin probe. B16F10 cells were treated with heat $\left(42^{\circ} \mathrm{C}\right)$ or with the non-proteotoxic membrane fluidizer benzyl-alcohol $(40 \mathrm{mM})$ for 1 hour, then labeled with BODIPY FL C5 sphingomyelin and imaged by an Olympus

Fluoview confocal microscope. The data shown are mean values, $\pm S E M, n=3$. 
In order to further investigate the effect of fluidization on the fine structure of the membrane we applied a fluorescent lipid probe, BODIPY FL C5-sphingomyelin to the membrane. The specificity of lipidated BODIPY probes in the detection of membrane microdomains was already demonstrated by former studies ${ }^{211}$. When added to live mammalian cells, BODIPY FL C5-sphingomyelin was distributed exclusively within the plasma membrane and labelled the sphingomyelin rich domains. Thus by the detection of the probe fluorescence it was possible to measure the size distribution of these domains. In the present study, B16F10 cells treated with $40 \mathrm{mM}$ BA or exposed to heat stress at $42^{\circ} \mathrm{C}$, were subsequently incubated with BODIPY FL C5-sphingomyelin. The labeled "spots" were visualized by confocal microscopy and the sphingomyelin probe-labelled domains were sorted into six classes according to their diameters. Whereas the number of smaller domains decreased in response to both heat and BA priming, the larger domains accumulated (Fig. 2.). Importantly, the amplitude of the effects observed was always more pronounced in the case of BA induced fluidization.

\subsection{Membrane fluidization mediated membrane domain rearrangement is coupled with increased HSP expression}

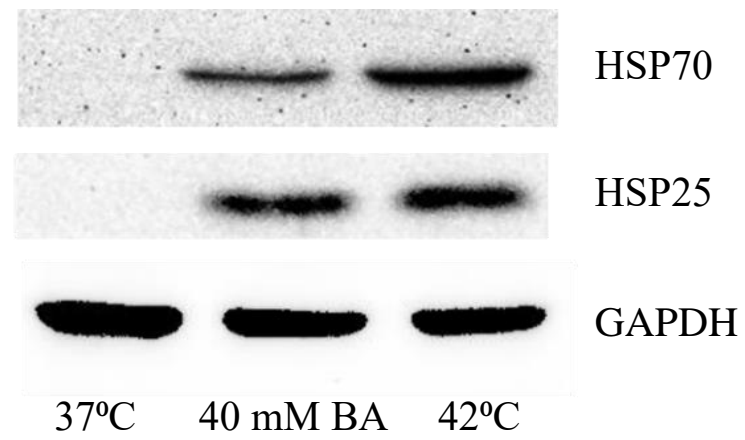

Figure 3. The effects of heat shock-induced or BA-induced membrane fluidization on HSP expression in $\mathrm{B} 16 \mathrm{~F} 10$ cells. B16F10 cells were treated with heat $\left(42^{\circ} \mathrm{C}\right)$ or with the nonproteotoxic membrane fluidizer benzyl-alcohol $(40 \mathrm{mM})$ for 1 hour, then after a 16 hour incubation period their protein content was analyzed by western blotting.

In order to evaluate further similarities between heat and BA treatment we have assayed for the most well-known cellular response to heat, the induction of heat shock proteins. The increased level of membrane fluidity, in parallel with the reorganization of 
microdomains, led to a significant increase in the levels of HSP70 and HSP25 proteins in B16F10 cells detected by western blotting (Fig. 3.). Thus, our experiments were able to provide evidence for the downstream effect of membrane fluidization on gene expression. The synthesis of the HSP70 protein upon membrane fluidization was already described by a previous study in mammalian cells ${ }^{210}$. We were not only able to recapitulate this phenomena in this study but, also to show a distinct upregulation of the HSP25 protein for the first time upon BA induced membrane fluidization. In general the effect of BA was not comparable to the heat treatment in the manner of HSP induction, as the detected HSPs were more pronounced upon heat stress.

\subsection{The membrane fluidization induced HSP expression translates into the acquisition of thermotolerance}

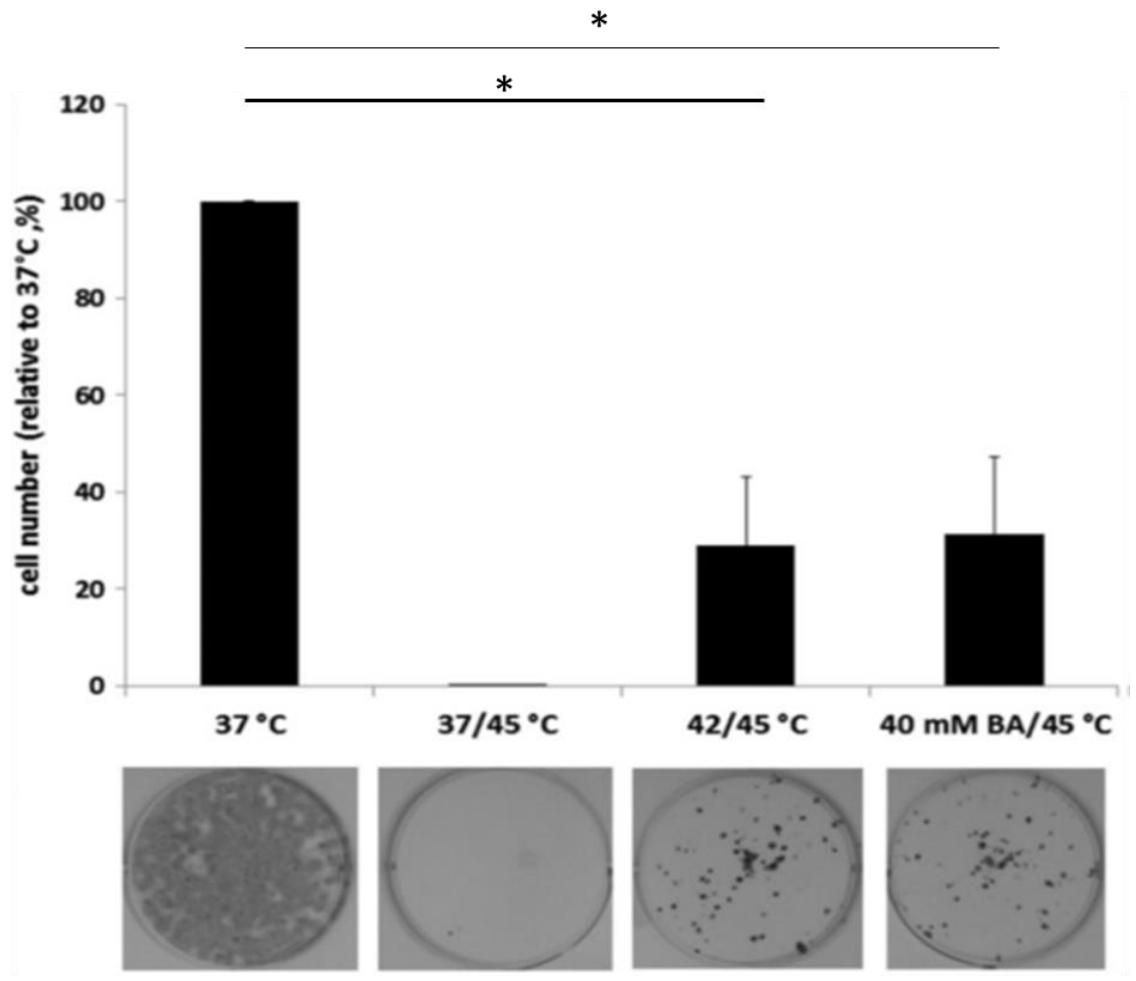

Figure 4. Acquisition of thermotolerance with heat and BA. B16F10 cells were pre-treated with heat at $42^{\circ} \mathrm{C}$ or with $40 \mathrm{mM} B A$ for 1 hour. The preconditioning effect of the treatments was assessed by subjecting the cells to a lethal heat shock at $45^{\circ} \mathrm{C}$. After a 12 day recovery period the cells were fixed in $4 \%$ paraformaldehyde for 10 minutes, and stained with a $0.5 \%$ crystal violet solution for 20 minutes. Cellular survival was assessed by counting the colonies. The data shown are mean values, $\pm S E M, n=6 .{ }^{*} P \leq 0.005$ according to a testing procedure using the Student $t$ test. 
In order to analyze if the expression of the HSPs are translated into a physiological relevant response, we have investigated the effects of the administration of BA and heat priming on thermotolerance development. Thermotolerance can be described as the tolerance of a cell to thermal damage which can be dramatically increased by prior heat conditioning. Thermotolerance was assessed by the preconditioning of B16F10 cells for 1 hour $\left(40 \mathrm{mM} \mathrm{BA}\right.$ at $37^{\circ} \mathrm{C}$ or $\left.42^{\circ} \mathrm{C}\right)$, followed by 1 hour of lethal heat stress treatment at $45^{\circ} \mathrm{C}$ after a 16 hour recovery period. The different groups of cells were plated into cell culture dishes by limited dilution and the growth of individual colonies were assessed after 2 weeks. When preconditioned either with heat shock or with BA, the cells acquired a highly elevated thermotolerance which can be vaguely understood as a general resistance to stress conditions (Fig. 4.).

\subsection{The artificial expression of HSP25 buffers the fluidizing effect of benzyl alcohol}

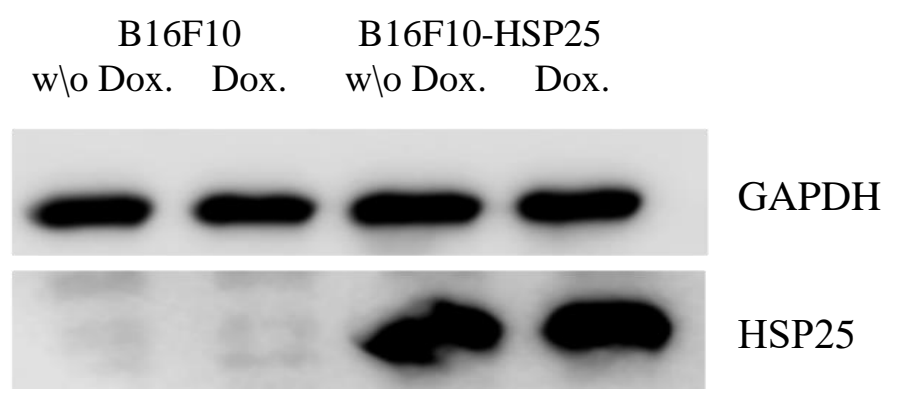

Figure 5 A). HSP25 protein expression in B16F10 and B16F10-HSP25 cells. $B 16 F 10$ and B16F10-HSP25 cells were either left untreated or were treated with doxycycline (Dox.) for 24 hours, then their protein content was analyzed by western blotting. 


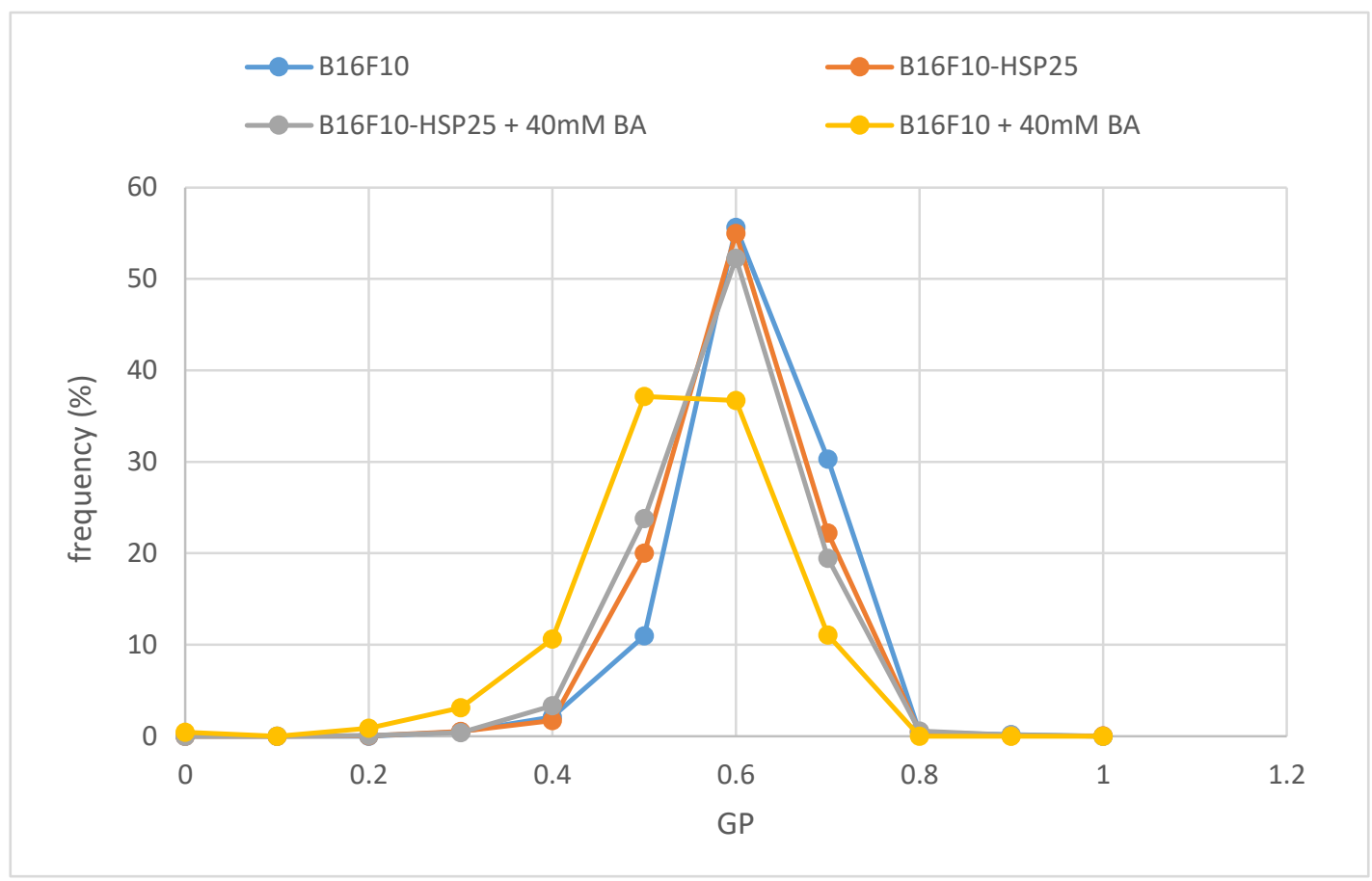

Figure $5 \mathrm{~B}$ ). GP value changes in $\mathrm{B} 16 \mathrm{~F} 10$ or B16F10-HSP25 cells upon BA treatment. B16F10 and B16F10-HSP25 cells were left untreated or treated with $40 \mathrm{mM}$ BAfor 1 hour.

Membrane order was assessed by the addition of the membrane incorporating dye di-4ANNEPDHQ. Data is expressed as GP distribution of at least 80 PM segments. Data is shown as a frequency distribution.

Considering the above suggested importance of the membrane structure in stress tolerance, we further sought to investigate if the presence of the detected HSP25 affects the plasma membrane order. For this reason we used B16F10 cell lines artificially expressing the HSP25 protein under an inducible tetracycline operator regulated expression cassette (Fig. 5. A). Our experimental setup using the cell lines mentioned above was similar to one seen in Fig. 1. The stable expressing clones were subjected to a treatment with $40 \mathrm{mM} \mathrm{BA}$, and subsequently stained with the membrane incorporating di-4-ANEPPDHQ dye to assess the physical state of the membrane. The addition of BA induced membrane fluidization in our control cells. However, surprisingly in HSP25 expressing cells BA treatment was not resulted in membrane fluidization (Fig 5 B). In these cells the membrane showed the same ordered state as before BA treatment, suggesting a possible buffering effect of HSP25 against perturbations causing membrane structural changes. Surprisingly, the non-induced B16F10-HSP25cell has shown the same phenomena without the addition of doxycycline, an agent responsible for the induction of 
the artificial gene expression in this system. Although, after the analysis of HSP25 protein content of B16F10-HSP25 cells by western blot and immunofluorescence, it has been revealed that these cells contain a considerable amount of HSP25 even under the non-induced conditions (Fig. 5 A). This phenomena could be originating from the nature of the expression cassette running the artificial HSP25 expression, as it is prone to show the effect of so called leakage of gene expression. Which means in practical terms that the artificially introduced gene is expressed under basal conditions at a certain level. This result could explain the effect of BA treatment on membrane order of the non-induced B16F10-HSP25 cells considering that HSP25 was present in the system in amount comparable to that found in induced cells. These experiments (Fig 5 B) are pointing out a critical function for HSP25 which could be very similar to the observed effect of other small HSPs on the membrane lipid order ${ }^{62}$. Therefore the data above suggests a key role for HSP25 in the maintenance of membrane homeostasis under membrane perturbing stress conditions.

4.6 The expression of HSP25 alters the membrane domain structure and affects the redistribution of membrane microdomains

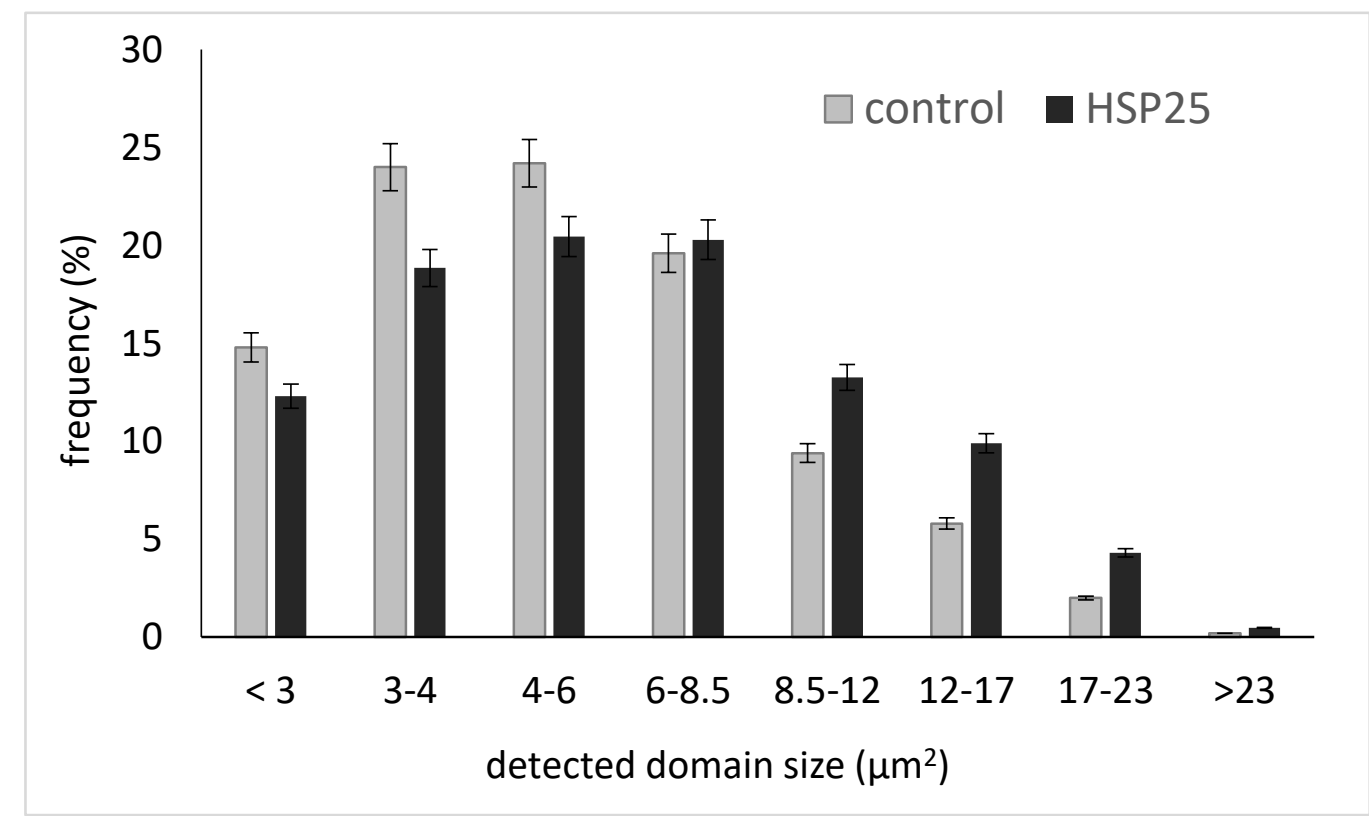

Figure 6. Size distribution of membrane microdomains upon HSP25 expression. Redistribution of the sphingomyelin membrane domains on the surface of B16F10 cells, was monitored by a BODIPY FL C5 sphingomyelin probe. B16F10 or B16F10-HSP25 cells were labeled with BODIPY FL C5 sphingomyelin and imaged by an Olympus Fluoview confocal microscope. The data shown are mean values, $\pm S E M, n=3$. 
In order to decipher the nature of HSP25`s action on the membrane and to connect this data to our previous observations, we again set up an experiments to analyze the size distribution of membrane domains but now in the presence of HSP25. We have labelled the membrane of wild type and HSP25 expressing B16F10 cells with the BODIPY FL C5 sphingomyelin probe as in Fig 2. Considering that the B16F10-HSP25 cells showed a very similar HSP25 expression under either induced or non-induced conditions, which level was comparable to that found after heat or BA treatment, we have chosen to proceed with the non-induced cells.
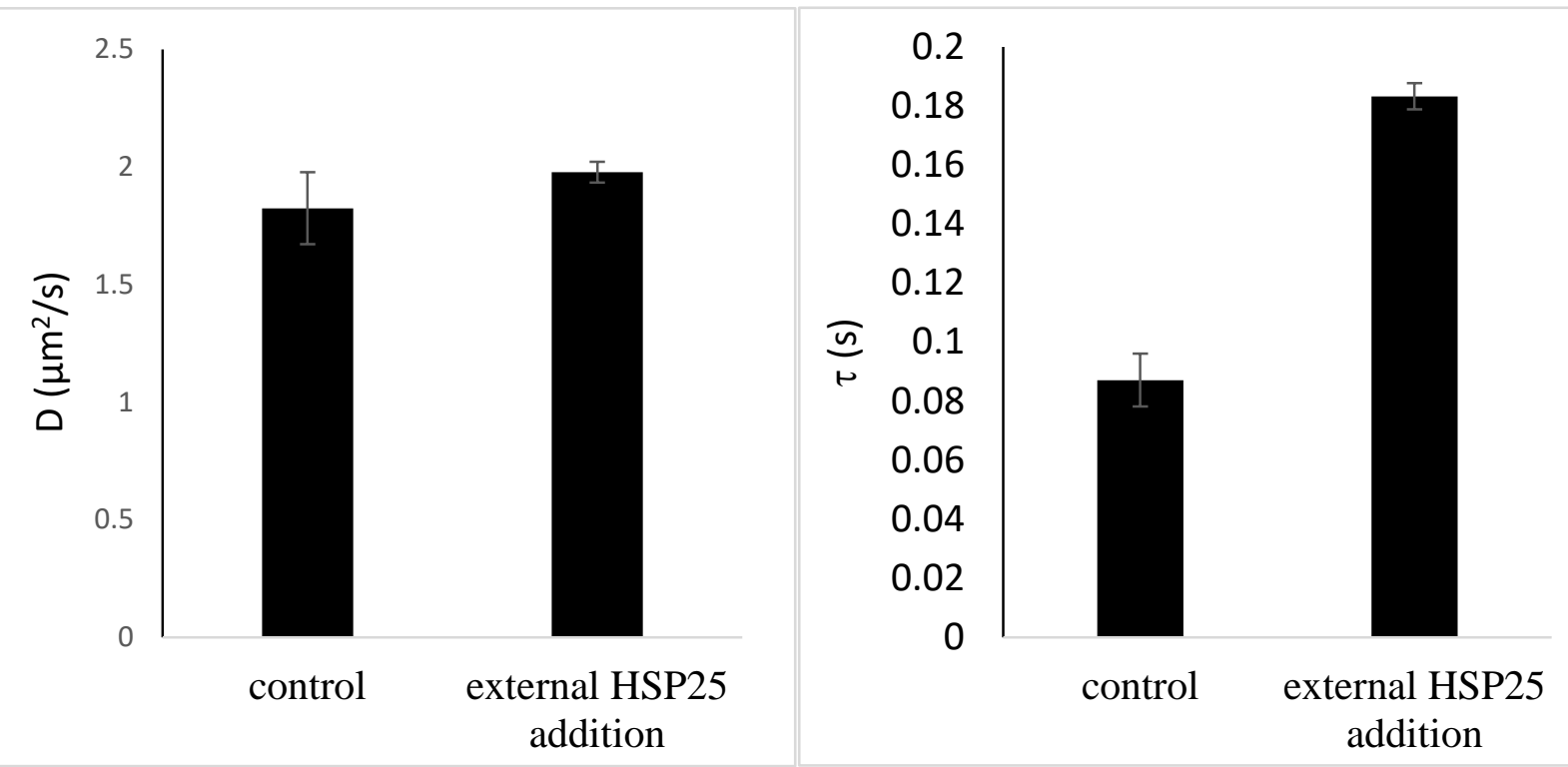

Figure 7. Fluorescence correlation spectroscopy measurements on isolated cell membranes. Membrane sheets were prepared from B16F10 cells which were subsequently stained with the STAR488-PEG-cholesterol probe. Purified HSP25 was externally added to the sheets in PBS containing $1 \%$ BSA. The data shown are mean values, $\pm S E M, n=3$.

In order to further determine the mechanism behind this effect we decided to go one level deeper in the analysis of the membrane structure. We approached this by performing fluorescent correlation spectroscopy measurements on isolated plasma membrane sheets from wild type B16F10 cells. At first, we got rid of all other elements of the cell except the cell the membrane during the preparation of the membrane sheets, purified HSP25 was externally added to the system in order to analyze its effect on the membrane 
structure. In this way we were able to work on a system where only the isolated plasma membrane was present without the other constituents of the cell and to analyze if HSP25 exerts its action through a direct interaction with the membrane. After the preparation of the plasma membrane sheets we labelled them with an STAR488-PEG-cholesterol probe and the probe fluorescence was visualized with single particle imaging microscopy. With this approach we were able to measure the fluctuation of fluorescence intensity of pixels caused by the movement of the probe within the plane of the membrane and calculate two parameters from this data: the diffusion constant $(D)$ and the confinement time $(\tau)$. These parameters describe the lateral diffusion of the probe and the organization of the membrane, respectively. Zero $\tau$ values correspond to free diffusion while positive $\tau$ means confined diffusion which reflects well the presence of membrane structural barriers which confine the probe to a certain location, like membrane microdomains. The fluorescent spectroscopy measurements performed on the membrane sheets revealed key characteristics of the nanoscale membrane structure (Fig. 7.). Very intriguingly the presence of HSP25 did not alter the general diffusion constant of the probe hence the overall fluidity of the total membrane. However, the confinement values showed a distinct change upon the addition of HSP25. As it has been described earlier that the confinement values reflect the compartmentalized nature of the membrane ${ }^{212}$. This observation regarding the higher confinement in the presence of HSP25 was in line with our data on the higher membrane domain sizes upon HSP25 expression. 
4.7 Membrane proteins as central players in cancer progression:

PRL3 expression is upregulated upon exposure to genotoxic stress

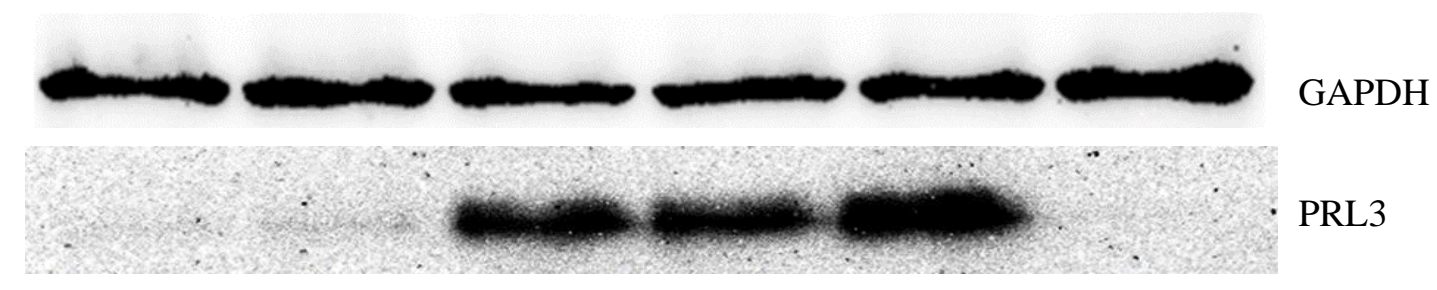

CONTROL CONTROL CISPLATIN ETOPOSIDE DOXORUBICIN PACLITAXEL (DMSO)

Figure 8. A) PRL3 expression upon treatment with genotoxic anti-cancer agents in B16F0 cells. B16F0 cells were treated with $5 \mu \mathrm{M}$ etoposide, $50 \mu \mathrm{M}$ cisplatin, $2.5 \mu \mathrm{M}$ doxorubicin, and $2 \mu \mathrm{M}$ paclitaxel for 24 hours. The protein content of the cells was analyzed by western blotting.

GAPDH

PRL3
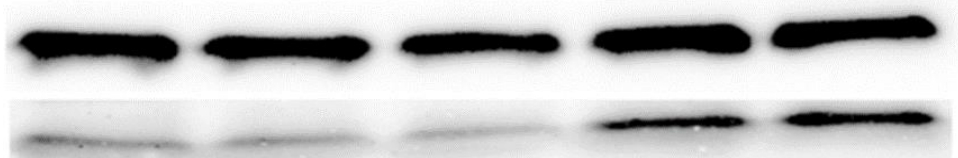

CISPLATIN
CONTROL $\quad 1 \mu \mathrm{M}$

$50 \mu \mathrm{M} \quad 100 \mu \mathrm{M}$

Figure 8. B) PRL3 expression dose curve upon cisplatin administration in $\mathrm{B} 16 \mathrm{FO}$ cells. $\mathrm{B} 16 \mathrm{FO}$ cells were treated with the indicated amounts of cisplatin for 24 hours. The protein content of the cells was analyzed by western blotting.

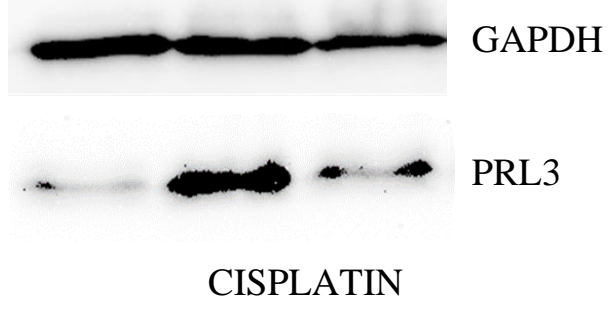

CONTROL $24 \mathrm{~h} \quad 48 \mathrm{~h}$

Figure 8. C) PRL3 expression time curve upon cisplatin administration in $\mathrm{B} 16 \mathrm{FO}$ cells. B16F0 cells were treated with $50 \mu M$ cisplatin. PRL3 expression was analyzed 24 hours after treatment or after a second 24 hour incubation period without treatment by western blotting.

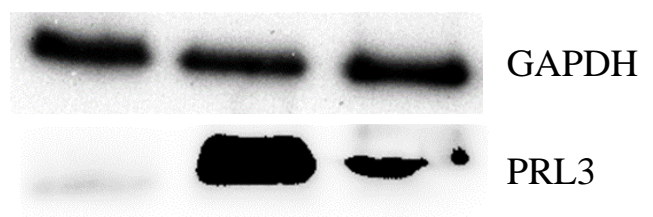

DOXORUBICIN

DOXORUBICIN

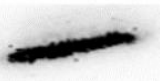

PRL3

$1 \mu \mathrm{M} \quad 2.5 \mu \mathrm{M} \quad 5 \mu \mathrm{M}$

Figure 8. D) PRL3 expression dose curve upon doxorubicin administration in $\mathbf{B 1 6 F 0}$ cells. B16FO cells were treated with the indicated amounts of doxorubicin for 24 hours. The protein content of the cells was analyzed by western blotting $(n=3)$. 


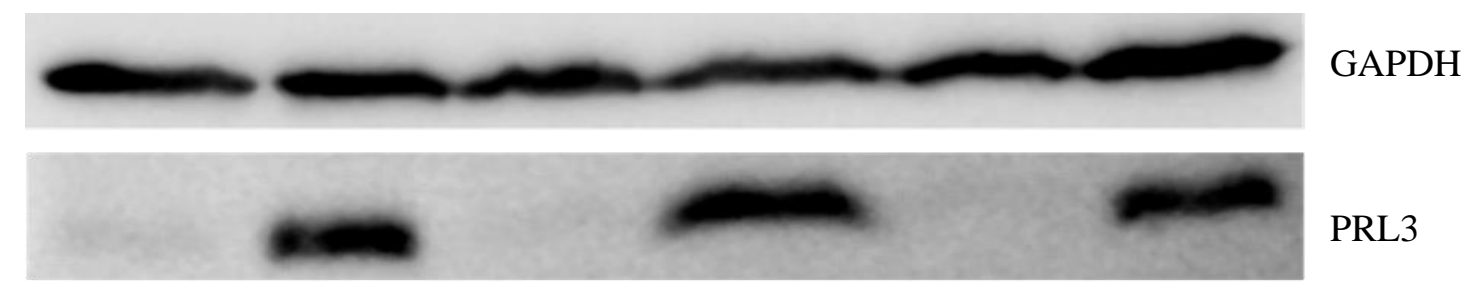

CONTROL DOXORUBICIN CONTROL DOXORUBICIN CONTROL DOXORUBICIN
$\mathrm{B} 16 \mathrm{~F} 0$
$\mathrm{B} 16 \mathrm{~F} 1$
$\mathrm{B} 16 \mathrm{~F} 10$

Fig 8. F) PRL3 upregulation upon drug administration is the characteristic feature of B16F0, B16F1 and B16F10 cells. The three B16 cell lines were treated with $2.5 \mu \mathrm{M}$ doxorubicin for 24 hours. The protein content of the cells was analyzed by western blotting.

Since key observations has been made with B16 melanoma cells concerning PRL3`s oncogenic action and membrane localization ${ }^{148,213,214}$, we chose to undertake our studies with these cells as well. As we were primarily interested in the phenomena of early cancer development we chose particularly the B16F0 cell line from the set of B16 cells because the F0 cells are the closest to the original primary tumor from all the various B16 cell lines ${ }^{215}$. Despite the data available in the literature, PRL3 protein expression in the B16F0 cells was under the detection limit in our experiments ${ }^{216}$ (Fig. 8 A). In the light of the previously established connection between elevated PRL3 expression in noncancerous $\mathrm{MEF}{ }^{128}$ cells exposed to genotoxic carcinogens we sought to find out if such phenomena could be detected in the B16F0 cells as well. In order to keep our study as clinically relevant as possible we applied genotoxic stress conditions in the form of classical anti-tumor drugs with a well described effect on genomic DNA. Our studies consequently revealed a distinct expression induction of PRL3 in the B16F0 cells upon the exposure to drugs as doxorubicin, cisplatin and etoposide (Fig. 8 A). However, the application of a drug like paclitaxel, which acts through the inhibition of microtubule organization did not induced the expression of PRL3 (Fig. 8 A). Dose and time dependency was further verified with doxorubicin and cisplatin in B16F0 cells (Fig. 8 B, C, D, and E). With these data we are the first to show the induction of PRL3 protein in a cancer cell upon exposure to these therapeutic agents. These experiments might explain former observations describing the inhibition of PRL3 as a potent adjuvant to cisplatin based therapy in lung cancer ${ }^{121}$ or the negative effect of the artificial expression of PRL3 on doxorubicin treatment of acute myeloid leukemia ${ }^{122}$. The potential clinical importance of PRL3 induction upon these anti-cancer agents is underlined by the fact that we 
administered the drugs close to their published IC50 values ${ }^{217-219}$. These data are in line with previous observations describing an upregulation of PRL3 expression in cancer cells isolated from a DNA damaging carcinogen treated colon of mouse, and connected the presence of with an increased tumor initiation ability ${ }^{124}$.

Next, we sought to analyze if the same phenomena of PRL3 induction is present in other cell lines. Therefore, other members of the B16 melanoma family were treated with the indicated amounts of doxorubicin. PRL3 induction was shown to be characteristic for all of the three commercially available B16 cell lines, as the B16F0, B16F1 and the B16F10 cells were all showed a marked PRL3 expression upon treatment (Fig. 8 F). This is particularly surprising since PRL3 mRNA induction was described in all of these cell lines upon treatment with the genotoxic compound phorbol ester phorbol 12-myristate 13-acetate (PMA) ${ }^{220}$. 
4.8 B16 cells show enhanced growth properties upon PRL3 expression

B16F0 B16F0 B16F0-PRL3

(MOCK)

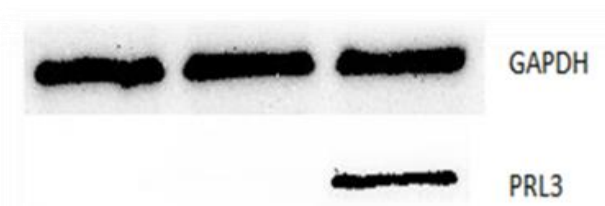

$\mathrm{B} 16 \mathrm{~F} 0$

wlo Dox. Dox.
B16F0-PRL3

wlo Dox. Dox.

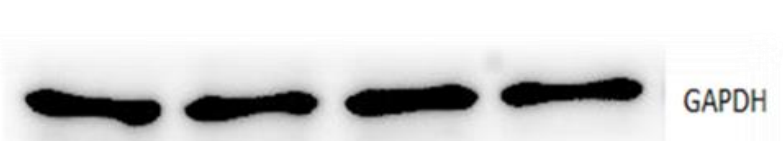

PRL3

Figure 9. A) Expression of human PRL3 in B16F0 cells a) either by constitutive or b) conditional manner. $B 16 F 0, B 16 F 0-M O C K, B 16 F 0-P R L 3$ (constitutive expression) or B16F0-PRL3 (inducible expression) cells were either left untreated or were treated with doxycycline for 24 hours, then their protein content was analyzed by western blotting.

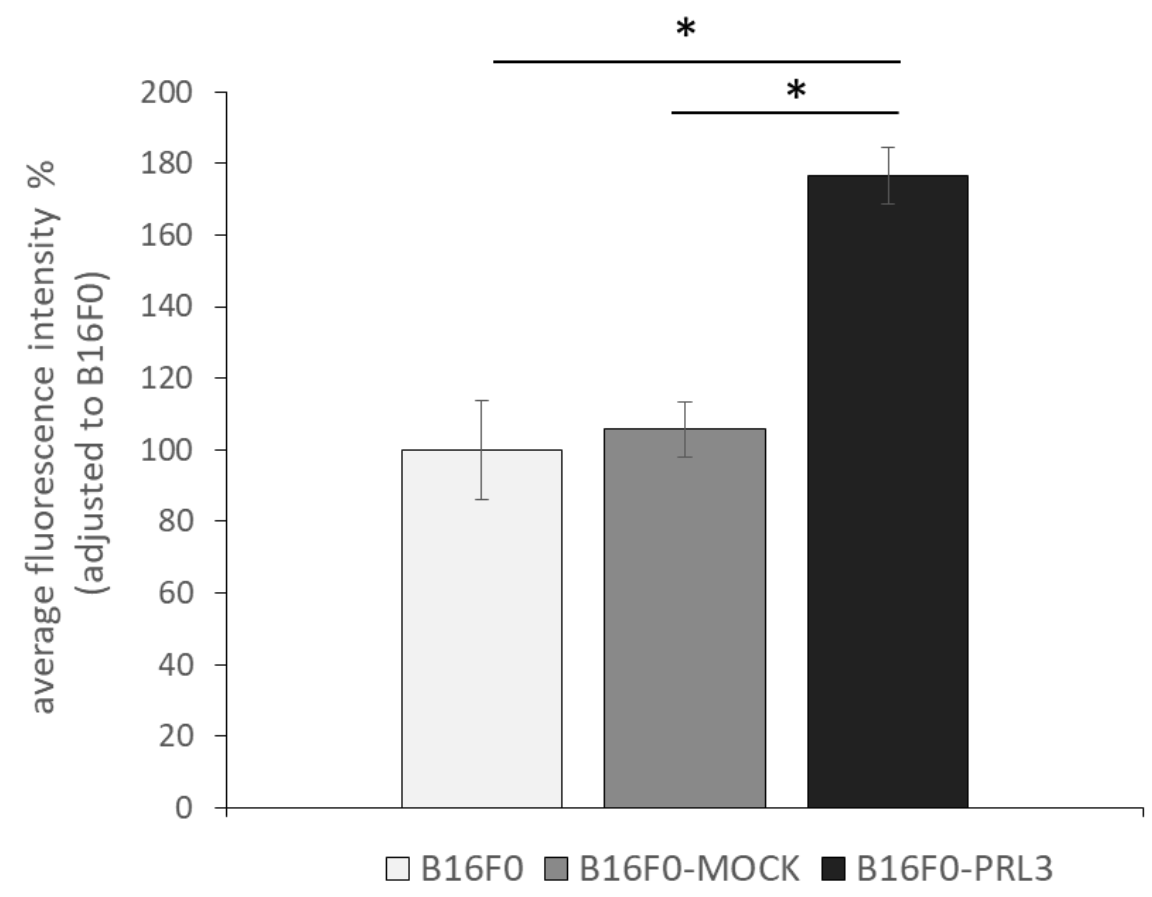

Figure 9. B) The growth of B16F0-PRL3 cells in anchorage independent conditions. The indicated B16FO cells were seeded into agar-containing media and cultured for 14 days. Colony formation from single cells was assessed by measuring cell viability with a resazurin-based Alamar blue assay The data shown are mean values, $\pm S D(n=3)$. $* P \leq 0.005$ according to a testing procedure using the Student $t$-test. 


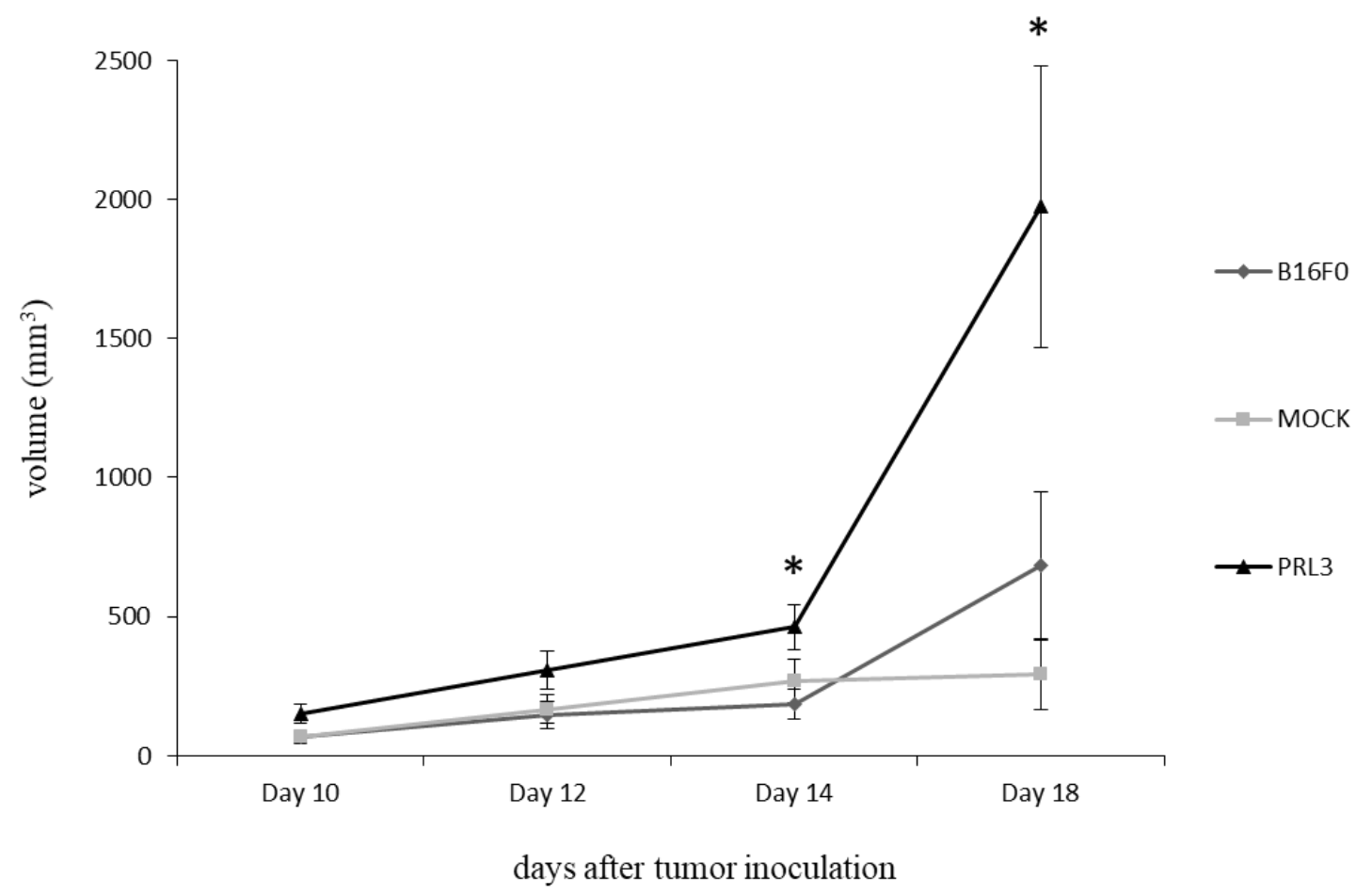

Figure 9. C) Tumor volumes changes of subcutaneously injected B16F0 cells in mice. The indicated sets of cell were injected subcutaneously into C57BL/6J mice $(n=6$, per group, \pm SEM). The tumor volumes were measured at the indicated time points. $* P<0.05$ according to a closed testing procedure using the Aspin-Welch t-test, compared to B16FO.

In order to assess the possible benefits of the induction of PRL3 for a cancer cell we made an attempt to model this phenomenon by the artificial expression of (human) PRL3 in B16F0 cells. To this end we created cell lines expressing PRL3 either under a constitutive or an inducible promoter, where the induction is provided by a tetracyclinecontrolled expression system (Fig. 9 A). The human PRL3 protein sequence shows a high homology to the mouse sequence $(96.5 \%)$ where all the functional domains are $100 \%$ identical to each other. By using the human sequence in our experiments we sought to model the oncogenic effect of the human PRL3, similar to studies where the in vivo tumorigenicity of a human oncogene was verified by introducing it to a mouse system 
221,222. At first we analyzed the effect of PRL3 expression on anchorage dependent growth, by measuring the ability of these cells to establish three-dimensional colonies from a single cell in a soft agar assay. Our results showed a significant increase in single cell colony growth upon PRL3 expression (Fig. 9 B). This method is generally accepted to assess uncontrolled cell growth and since the spheroid colonies originate from a single cell it is considered to be a good model of in vivo tumor growth. Next, we aimed to test the validity of these data in an in vivo setting to evaluate primary tumor growth. In order to do that we injected the same set of cells subcutaneously into mice. The data we gained from these experiments were in line with our previous findings as the PRL3 expressing B16F0 cells established subcutaneous tumors with a bigger volume (Fig. 9 C). Previous findings from the literature are further supporting our data since PRL3 expression was connected with the tumor initiating cell population in colorectal carcinoma ${ }^{124}$. Furthermore non-physiological PRL3 expression was shown to induce stem cell like signatures in leukemia cells and corresponded with the presence of a leukemia initiating cells in the population ${ }^{125}$. Therefore, it is plausible that PRL3`s action is realized in cancer cells by affecting tumor initiation thereby influencing the outcome and relapse rate of cancer.

4.9 PRL3 associates with the plasma membrane and localizes to the caveolae

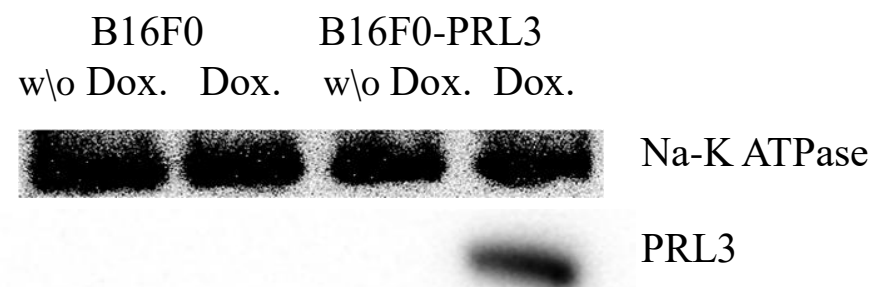

Figure 10. A) Analysis of PRL3 localization by plasma membrane isolation. $B 16 F O$ or B16F0-PRL3 cells were left untreated or treated with doxycycline (Dox.). The cells were subjected to total membrane isolation. The protein content of the total membrane fractions was analyzed by western blotting. The transmembrane ion channel, sodium-potassium ATPase was used as a loading control. 


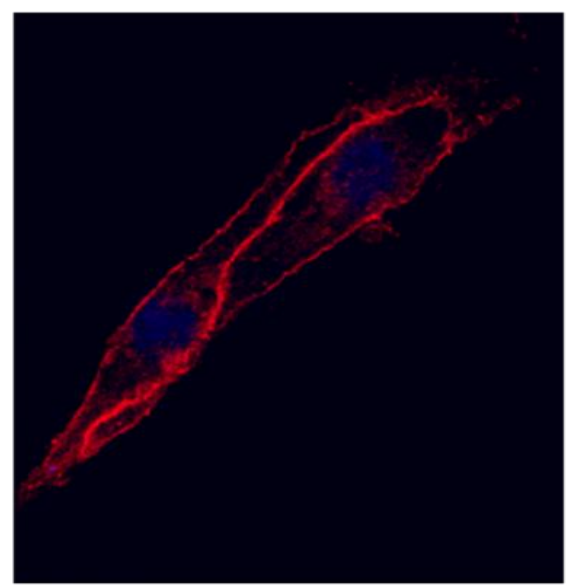

RED: PRL3

BLUE: DAPI

Figure 10. B) Analysis of PRL3 localization by confocal imaging. B16F0-PRL3 cells were induced by doxycycline and stained with an anti-PRL3 antibody. DAPI was used as a counter stain.

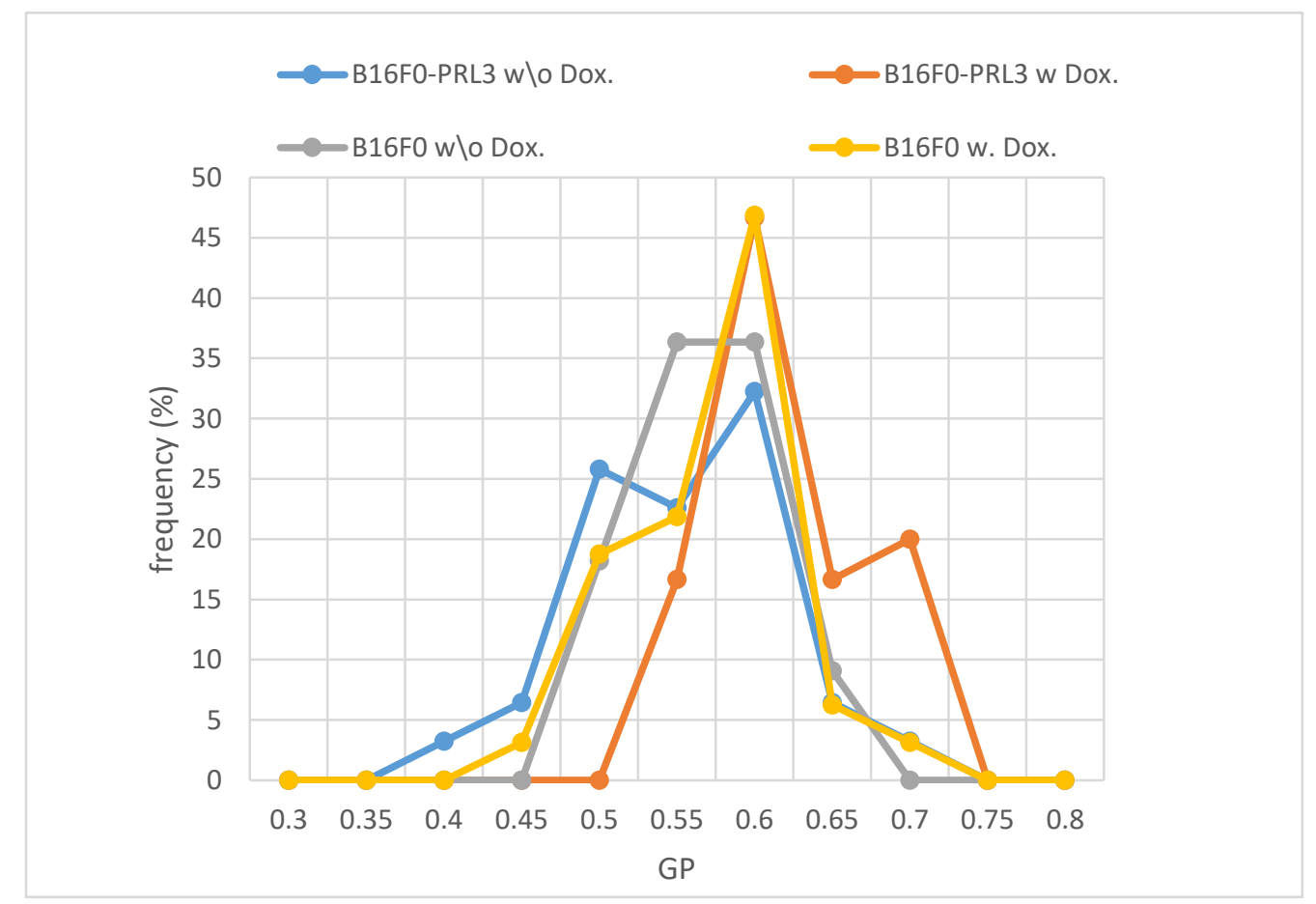

Figure 10. C) GP value changes upon PRL3 expression. B16F0 or B16F0-PRL3 cells with an inducible PRL3 expression were left untreated (w/o Dox.) or treated with doxycycline (w. Dox.) Membrane order was assessed by the addition of the membrane incorporating dye di-4ANNEPDHQ. Data is expressed as GP distribution of at least 80 PM segments. Data is shown as a frequency distribution. 
FRACTION NUMBER:
$\begin{array}{llllllllllll}\text { 1. } & 2 . & 3 & 4 . & 5 . & 6 . & 7 . & 8 . & 9 . & 10 . & 11 . & 12 .\end{array}$
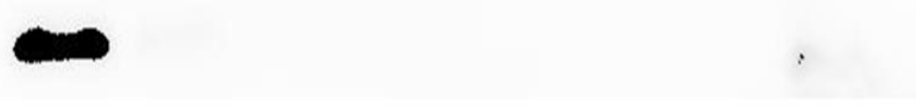
Cav1

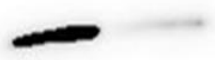
PRL3

Figure 10. D) PRL3 localization to the Caveolin 1-rich submembrane fraction in doxycycline-induced B16F0-PRL3 cells. B16FO-PRL3 cells were induced with doxycycline, then subjected to plasma membrane raft isolation. 13 raft fractions were collected and analyzed by western blotting. An anti-Cavl antibody was used to mark the localization of the caveolar domains within the fractions.

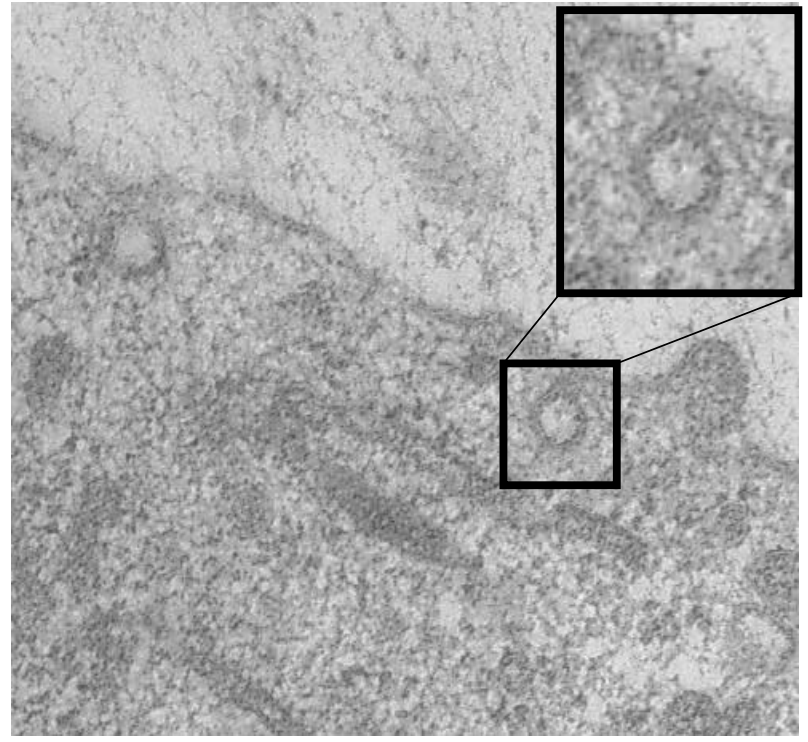

Figure 10. E)

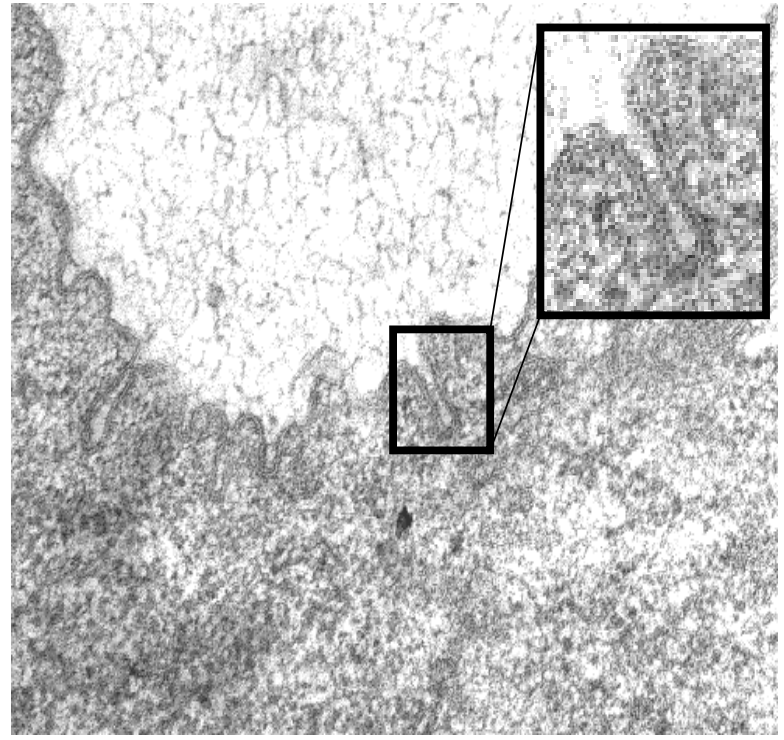

Figure 10. F)

Figure 10. E, F) Representative TEM images of the membrane of B16F0-PRL3 cells with or without doxycycline induction. The cells were fixed in $1 \%$ osmium tetroxide and $1 \%$ glutaraldehyde solution. The cell pellet was solidified on ice and cut into small blocks. Ultrathin sections were contrast-stained with uranyl acetate and lead citrate. (E) non-induced B16FOPRL3 cells show an intact caveolae morphology (resolution: 15000X), (F) PRL3-expressing B16F0 cells show elongated caveolae-like membrane formations (resolution: 40000X).

Caveolae-like structures are indicated with arrows. 
Considering that our data connected PRL3 to a distinct phenotype which might promote tumor growth, we sought to decipher the functional background of PRL3's action. At first we analyzed PRL3's membrane localization taking into account how crucial it seems to be for PRL3's oncogenic function ${ }^{148}$. Our results were verifying PRL3's membrane localization in the $\mathrm{B} 16 \mathrm{~F} 0$ cells by the analysis of isolated plasma membrane fractions (Fig. 10. A) or by confocal imaging (Fig. 10. B). Next, we concentrated our efforts to evaluate the nature and membrane environment of this interaction. At first we have analyzed the general plasma membrane structure by the addition of a membrane incorporating dye di-4-ANEPPDHQ which is intercalating into the lipid bilayer and changes its emission spectra according to the lipid packing order of the membrane, as it has been detailed earlier. According to these results PRL3 expression changes the overall plasma membrane structure towards a more ordered, rigid state (Fig. 10. C). This data indicates a PRL3-mediated effect on membrane organization and suggests that the PRL3associated oncogenic phenotype could be better defined through a deeper analysis of the membrane. For this reason, we focused on determining the submembrane localization of PRL3. Our experiments revealed that the detergent-resistant, caveolae-type membrane microdomain region of the plasma membrane was a primary localization site for PRL3 (Fig. 10. D). The detection of the classical flask-shaped caveolae structures at the membrane of B16F0 cells (Fig. 10. E) further support the localization of PRL3 in caveolae. However, it is necessary to note that in the PRL3-expressing cells we observed unusual elongated, tubular caveolae, different from the classical flask-shaped structures (Fig. 10. F). 


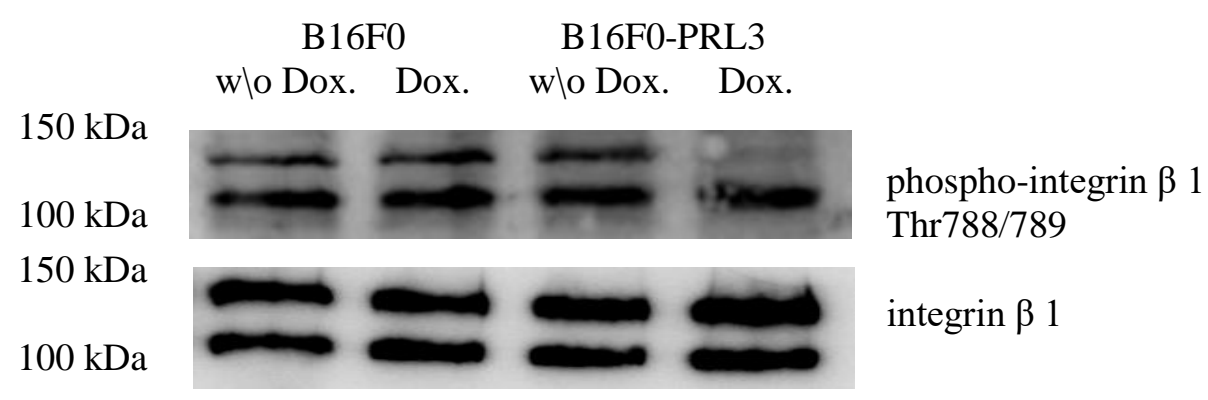

Fig 11. A) Dephosphorylation of Integrin $B 1$ at Thr788/789 upon PRL3 expression. B16F0 or B16FO-PRL3 cells with an inducible PRL3 expression were left untreated (w/o Dox.) or treated with doxycycline (w. Dox.) Subsequently integrin $\beta$ Iphosphorylation was analyzed by western blotting.

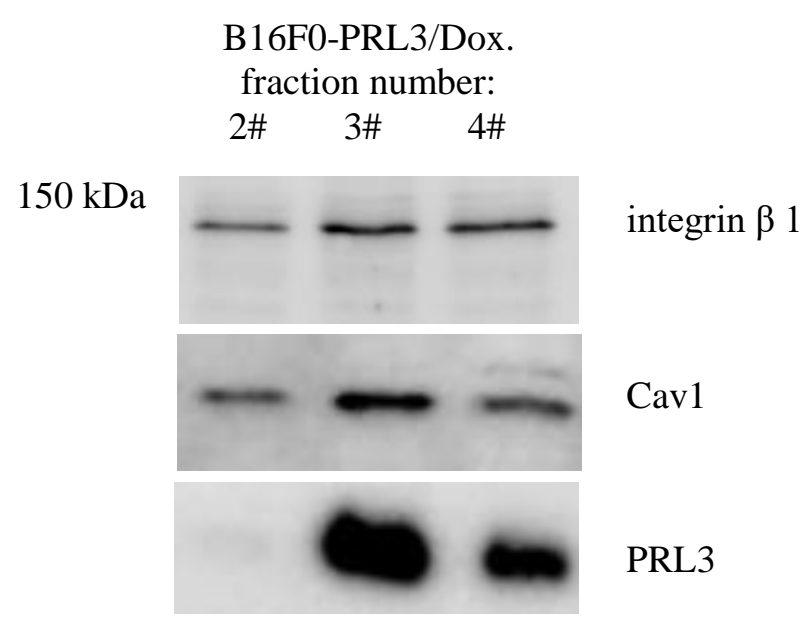

Fig 11. B) Localization of the higher mass integrin isoform within the caveolae fraction. B16F0-PRL3 cells were induced with doxycycline, then subjected to plasma membrane raft isolation. Raft fractions were collected and the fractions corresponding to the caveolae region of the membrane were analyzed for the presence of integrin $\beta 1$ by western blotting. An anti-Cavlantibody were used to mark the localization of the caveolar domains within the fractions. Only the $150 \mathrm{KDa}$ form of integrin $\beta 1$ is present in the caveolae membrane fraction and it cofractionates with PRL3.

With the knowledge at hand that PRL3`s action is most likely connected to the caveolae fraction of the membrane we were concentrating our efforts to characterize possible changes in the caveolae associated proteome upon PRL3 expression. We were especially 
interested in possible substrates for PRL3 that could explain the observed tumorigenic phenotype upon PRL3 expression in B16F0 cells. Following the logic outlined in the introductory chapter regarding the possible interaction partners of PRL3, we were particularly focusing on the integrin receptors. The facts that integrins are well known parts of the caveolar platforms and they are also described to be central in adhesion associated cell growth signaling ${ }^{31}$ made them an even more intriguing choice. PRL3 has been described in previous studies to interact with, effect the expression and localization of integrin receptors $152,153,155,163$. Our studies were able to verify the dephosphorylation of Integrin $\beta 1$ at Thr788/789 upon PRL3 expression (Fig. 11. A). Western blot experiments revealed a dual signal for integrin $\beta 1$, approximately at 100 and $150 \mathrm{KDa}$, similar to a previous observation which associated this phenomenon with different glycosylation patterns of the protein ${ }^{223}$. We could observe dephosphorylation only the of $150 \mathrm{KDa}$ form of integrin B1 (Fig.11. A). However, our raft fractionation experiments revealed that only the $150 \mathrm{KDa}$ form of the integrin $B 1$ was associated with caveolae in our system. The colocalization of integrin B1 and PRL3 in the plasma membrane was further verified by detecting both proteins in the same caveolae, membrane raft fraction (Fig. 11. B). The co-localization of the two proteins has been further verified by detecting both of them in the caveolae membrane raft fraction (Fig. 11. B). The Thr788/789 site of integrin $\beta 1$ has been associated with a dual function as the phosphorylation of this site was shown to be necessary for the assembly of adhesion complexes and the dephosphorylation is for the progress through the cell cycle. The studies describing these functions were associated with a phosphatase PP2A which catalyzed the dephosphorylation of integrin $\beta 1$ at this site and promoted the progression through the cell cycle. Considering that the phenotype we observed in the B16F0 cells could result from the de-phosphorylation of Thr788/789 we further analyzed the downstream effectors of PP2A in a hope to find similarities between the actions of the two phosphatases. The studies describing PP2A`s cell cycle regulatory role has associated PP2A with one key effector Rac1. Considering that the dephosphorylation of integrin $\beta 1$ by PRL3 at Thr788/789 could affect the pathway responsible for the regulation of Rac1 membrane localization and activation we made an attempt in our forthcoming experiments to analyze the Rac1 phenotype of B16F0 cells upon PRL3 expression. 
4.11 The presence of PRL3 alters the activation and plasma membrane targeting of Rac1

B16F0 wlo Dox. Dox. wlo Dox. Dox.

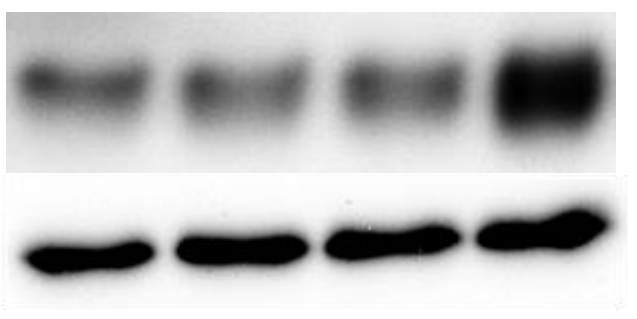

Rac1

(total)

Rac1

(plasma membrane)

PRL3

(plasma membrane)

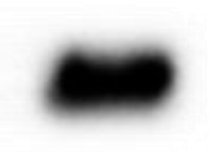

Figure 12. A) Rac1 plasma membrane localization. B16F0 or B16F0-PRL3 cells were left untreated ( $w / o$ Dox.) or treated with doxycycline (Dox.). The cells were subjected to total membrane isolation. The protein content of the total membrane fractions was analyzed by western blotting. Racl from the total lysate was used as a loading control.

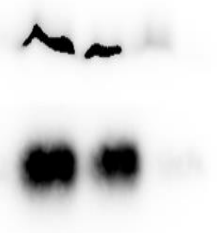

Cav1

Rac1

FRACTION NUMBER:

\section{2. 3. 4. 5. 6. 7. 8. 9. 10. 11. 12. 13.}

Figure 12. C) Rac1 localization to the caveolae fraction in B16F0-PRL3 cells. B16FO-PRL3 cells were induced with doxycycline, then subjected to plasma membrane raft isolation. 13 raft fractions were collected and analyzed by western blotting. An antiCavl antibody were used to mark the localization of the caveolar domains within the fractions.

B16F0-PRL3

wlo Dox.

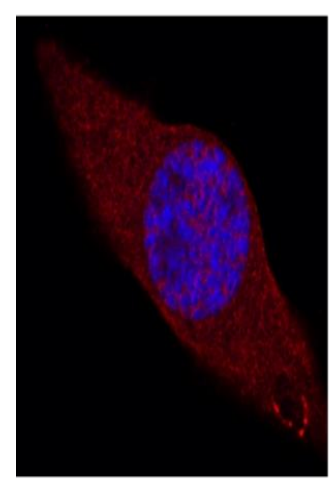

RED: Rac1

BLUE: DAPI

Figure 12. B) Representative images of Rac1 plasma membrane localization in PRL3expressing cells. $B 16 F 0-P R L 3$ cells were left untreated (control) or treated with doxycycline (Dox.). The cells were, stained with an anti-Racl antibody. DAPI was used as a counter stain.

\section{B16F0 B16F0-PRL3 wlo Dox. Dox. wlo Dox. Dox.}

IP: Rac1

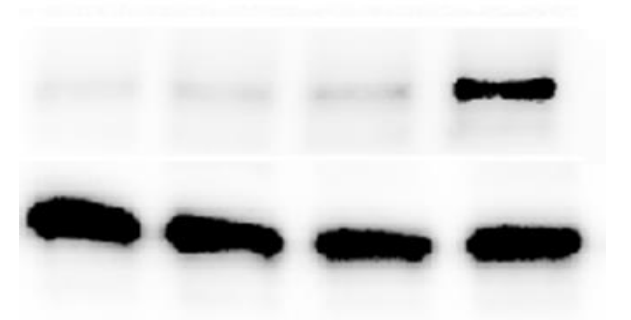

WB: Cav1

WB: Rac1
Figure 12. D) Rac1 coimmunoprecipitation with caveolin 1. B16FO or B16FO-PRL3 cells with an inducible PRL3 expression were left untreated (w/o Dox.) or treated with doxycycline (Dox.). Cells were lysed and subjected to coimmunoprecipitation with an anti-Racl antibody. Protein-protein interactions was assessed by western blotting. 
Rac1 activity and its plasma membrane localization to caveolae domains were shown to be central to the subsequent activation of signaling pathways regulating cellular growth. In cases where the balance of Rac1 plasma membrane targeting is impaired, cells acquire a higher capability to grow in non-adherent conditions by escaping the growth regulation connected to cellular attachment to a solid substrate. This control over cellular growth is primarily associated with integrins, the main sensors for the interaction with the cellular surroundings. Since Rac1 membrane localization was shown to be crucial for its activation, at first we analyzed its subcellular localization by confocal imaging and by comparing the ratio of Rac1 in plasma membrane isolates (Fig. 12. A, B). As our results identified an increased Rac1 plasma membrane targeting, we further analyzed Rac1 association with sub-membrane regions. Considering PRL3 localization to caveolae we have assayed for proteins preferentially located to this membrane compartment that could help Rac1 membrane targeting. The result of these experiments showed an increased direct physical interaction between Caveolin 1 and Rac1 (Fig. 12. D). Rac1 association with caveolae type lipid rafts has been further verified by localizing Rac1 into the caveolae fraction of the plasma membrane, similarly to PRL3 (Fig. 12. C). The enhanced localization of Rac1 to the plasma membrane could result in a proliferation advantage for PRL3 expressing cells by the disruption of a physiologically relevant cell growth regulatory pathway. 
4.12 Rac1 activation is connected with elevated levels of cyclin D1.

\author{
B16F0 B16F0-PRL3 \\ wlo Dox. Dox. wlo Dox. Dox. \\ Rac1-GTP \\ Cons
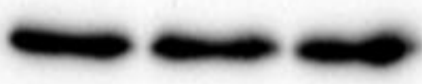 \\ Rac1 loading
}

\begin{tabular}{cccc} 
B16F0 & \multicolumn{2}{c}{ B16F0-PRL3 } & \\
wlo Dox. Dox. & wlo Dox. Dox. & IP: AKT
\end{tabular}

WB:

phospho-AKT

S473

WB:

AKT
Figure 13. A) Analysis of Rac1 GTP loading upon PRL3 expression. $B 16 F 0$ or $B 16 F 0-P R L 3$ cells with an inducible PRL3 expression were left untreated (w/o Dox.) or treated with doxycycline (Dox.). Cells were lysed and incubated with sepharose beads coupled with a protein which binds only to the Rac1-GTP molecule. The reaction was analyzed by western blotting. Racl was used as a loading control from the non-incubated fraction (Racl loading).
Figure 13. B) Analysis of AKT phosphorylation at Ser473 upon PRL3 expression. B16FO or B16F0-PRL3 cells with an inducible PRL3 expression were left untreated (w/o Dox.) or treated with doxycycline (Dox.). Cells were lysed and subjected to immunoprecipitation with an anti-AKT antibody. AKT phosphorylation was analyzed by western blotting.
B16F0 B16F0-PRL3

wlo Dox. Dox. wlo Dox. Dox.
B16F0 B16F0-PRL3

wlo Dox. Dox. wlo Dox. Dox.

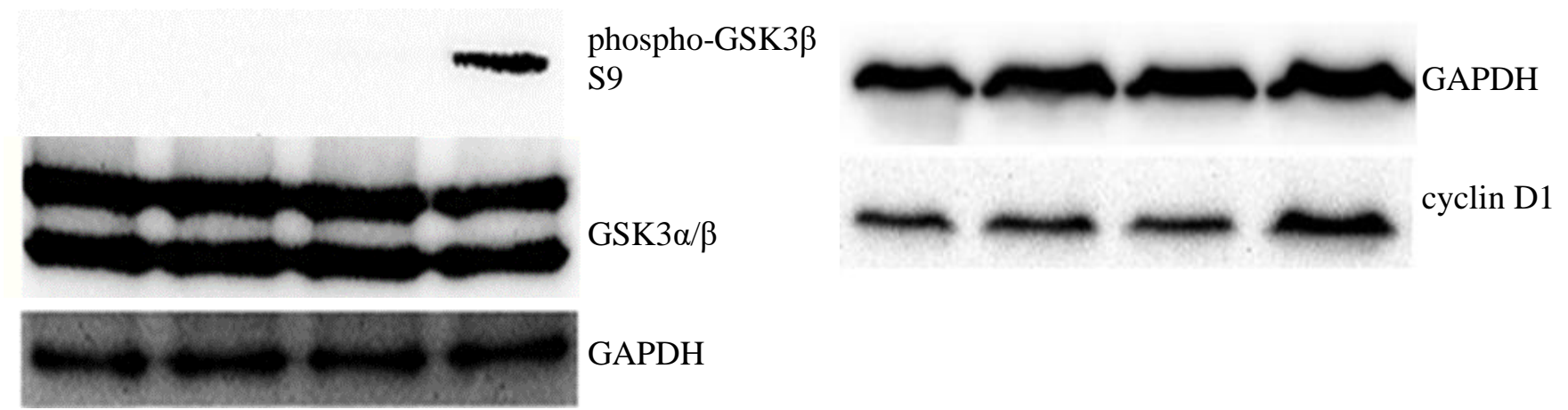

Figure 13. C) Analysis of GSK phosphorylation at Ser9 upon PRL3

expression. B16F0 or B16F0-PRL3 cells with an inducible PRL3 expression were left untreated (w/o

Dox.) or treated with doxycycline (Dox.). Subsequently GSK3 $\beta$ phosphorylation was analyzed by western blotting.

Figure 13. D) Analysis of cyclin D1 protein levels upon PRL3 expression. B16F0 or B16FOPRL3 cells with an inducible PRL3 expression were left untreated (w/o Dox.) or treated with doxycycline (Dox.). The protein content of the cells was analyzed by western blotting. 
wlo Dox.

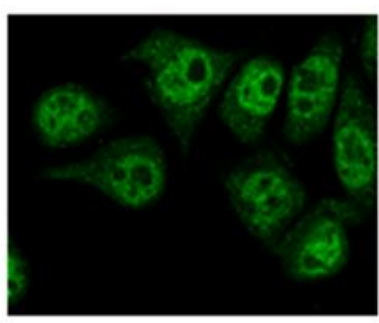

Dox.

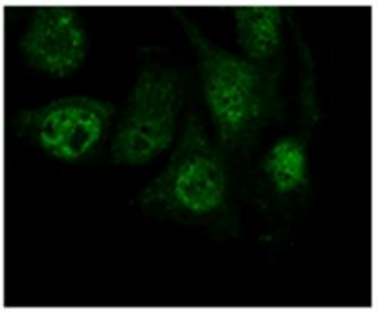

B16F0-PRL3

wlo Dox.

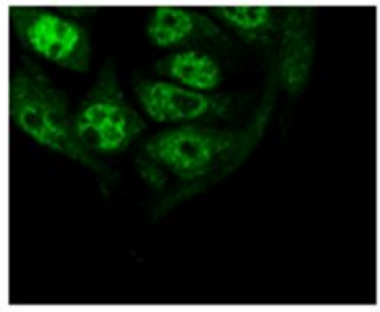

Dox.

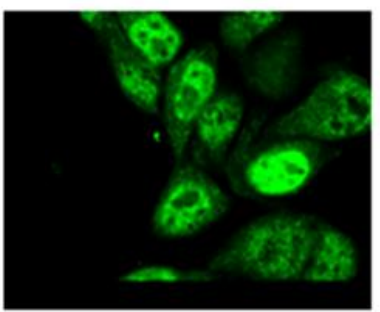

Figure 13. E) Representative confocal images of the nuclear localization of cyclin D1 in the indicated cells. B16F0 or B16F0-PRL3 cells with an inducible PRL3 expression were left untreated (w/o Dox.) or treated with doxycycline (Dox.). Cells were incubated with an anti-cyclin Dl antibody coupled with FITC.

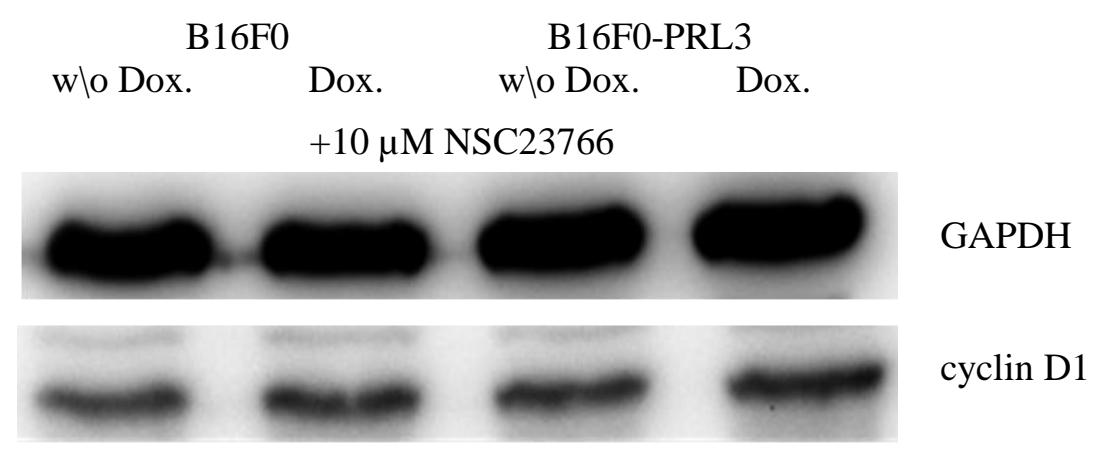

Figure 13. F) Rac1 inhibition decreases the elevated cyclin D1 levels. $B 16 F 0$ or $B 16 F 0$ PRL3 cells with an inducible PRL3 expression were left untreated (w/o Dox.) or treated with doxycycline (Dox.). The indicated cells were treated with $10 \mu \mathrm{M}$ NSC23766 for 24 hours in order to inhibit Rac1-dependent elevation of cyclin D1 levels. The protein content of the cells was analyzed by western blotting.

In order to characterize the signaling downstream of Rac1 which could account for the observed pro-tumorigenic phenotype, we have relied on the observations describing the Rac1 associated signaling in the context of cellular growth regulation. Rac1 GTP binding was shown to be a prerequisite for Rac1 cell cycle regulation. Therefore our primary aim was to identify these changes in our system upon PRL3 expression. Our data clearly showed a higher proportion of Rac1-GTP binding (Fig. 13. A). As such modulation of signaling has been further associated with the increased phosphorylation of the AKT kinase on the activator site at Ser473 in the context of cellular growth, we sought to analyze the phosphorylation changes at this amino acid residue. Our data has revealed a distinct AKT phosphorylation on this site which clearly indicates the activation state of the AKT kinase (Fig. 13. B). AKT takes part in anchorage dependent growth by the 
regulation of the GSK kinase, by inactivating it with phosphorylation at its Ser9 residue. Our studies revealed a strong presence of this inhibitory phosphorylation on GSK (Fig. 13. C). GSK is directly responsible for the regulation of the cell cycle by the fine tuning of the protein amounts of cyclin D1. GSK does this by targeting cyclin D1 to degradation by phosphorylating its Thr286 residue. Thus, we further sought to analyze the amounts and localization of cyclin D1 upon PRL3 expression. In these experiments we observed an elevated amount of cyclin D1 upon PRL3 expression (Fig. 13. D), the presence of which in the nucleus was verified by immunofluorescence staining (Fig. 13. E). The higher levels of cyclin D1 correspond well with the observed increased tumor growth. In order to clarify the role of Rac1 in the observed phenomena we inhibited Rac1 chemically by a small water soluble and cell permeable molecule NSC23766 ${ }^{180}$. This inhibitor has been described to inhibit the activation of Rac1 by interfering with the interaction of Rac1 and its GEFs. Furthermore NSC23766 was shown to be ineffective on other Rho GTPases such as Cdc42 or RhoA ${ }^{224}$. The inhibition Rac1 resulted in the drop of cyclin D1 to its original levels (Fig. 13. F). 


\section{Discussion}

Our understanding of how the plasma membrane functions has been fragmented for decades. As the first chapters of this thesis described, membrane researchers approached the membranes from very different angels and viewpoints since the early times. Physicists, chemist, biologists, computational and medical scientist still possess a short sightedness when trying to understand the complexity of membranes. This is originating from the fact that the plasma membrane is a complex system on its own. As such, it requires dedication and hard work to put together the many pieces of its lipid-protein puzzle, to gain a picture of a functioning membrane at the end. Many, multidisciplinary approaches emerged in the recent years as a response to this challenge. In this thesis we chose a scientific method which might be the closest to the heart of a biologist, since it originates back to the heroic times of the scientific history of biology. Namely, have a test subject, poke it, disturb it, provoke it, and then observe what happens with its behavior. Or in other words, analyze a complex system when thrown out of balance in order to understand its nature. During the design of the experiments of this thesis we chose two approaches according to this philosophy.

In a continuation of decades long work performed in our laboratory we decided to investigate the consequences of membrane fluidization on membrane domain organization and cellular stress response. We were particularly concentrating on the membrane associated roles of HSPs in membrane level stress management. With our second approach we hoped to gain better understanding on the role of the plasma membrane in pathological, specifically in cancerous conditions by studying the action of a membrane associated oncogene.

Thus, with these two sets of experiments we were trying to gain a deeper understanding on the physiology of the membrane under conditions when its balance is disturbed either by forced structural changes or by a pathophysiological stimuli. 
5.1 Structural membrane changes induced chaperon expression contributes to membrane homeostasis

The plasma membrane is compartmentalized into a mosaic of functional domains which are central to the regulation of processes associated with the cell surface. The reorganization of membrane microdomains at the plane of the membrane is a very distinct phenomena which is known to facilitate the initial transmission steps of environmental signals to downstream effectors. Initial studies showed a number of examples for such phenomena. The cholera toxin mediated crosslinking of the raft associated lipid, GM1 has resulted in the coalescence of small rafts into micrometer-sized domains ${ }^{225}$. The HIV-1 structural membrane associated protein, Gag was observed to induce coalescence of lipid rafts and hypothesized to aid the exit of virus particles out of the host cell ${ }^{226}$. The merger of growth factor receptor containing microdomains was also observed under growth factor stimulation ${ }^{227}$. Furthermore, physical forces as osmotic pressure generated membrane tension was also connected with the induction of large GM1 marked domains at the membrane 228 which were converted back almost immediately to smaller sized domains when the surface tension dropped back to physiological levels. The induction of the membrane associated heat shock response is also describing such a phenomena. So far the pathway components which are responsible for the induction of the membrane associated heat shock response and possibly localized into the coalesced domains are not characterized in detailed yet. However, a few effectors were already detected to take part in this phenomena. The transient receptor potential vanilloid (TRPV) receptor family was shown to play a role in the membrane level heat sensing. The TRPV membrane receptors can be activated by heat stress and could contribute to the induction of stress genes in plants and mammalian cells ${ }^{229}$. Other proteins as phospholipase $\mathrm{C}$ and phospholipase $\mathrm{A} 2$ were identified to promote the production of several lipid mediators upon heat and BA stress including the potent heat shock modulator, arachidonic acid ${ }^{230}$. Heat stress was observed to induce the membrane localization of an actin cytoskeleton organizing protein Rac1 in parallel with the activation of heat shock factor 1 (HSF1) ${ }^{231}$. Also the chemical inhibition of Rac1 was shown to decrease the heat induction of HSP transcripts ${ }^{187}$. However, considering the diversity and number of membrane proteins it is likely that these above mentioned examples are not the sole source of membrane stress signals. It is more likely that such induction of the cellular stress signaling machinery is associated with many modulators 
and effectors in the membrane in an organism and tissue specific manner. Other features of the membrane mediated stress signaling as the connection between membrane fluidization and the transcriptional induction of stress genes were also detailed by a previous study ${ }^{232}$, which is pointing out a central role for the transcription factor HSF1 in the transmission of the membrane associated stress signals to the nucleus. However, the presence and the extent of overexpression at the protein level was not yet determined for HSPs other than the HSP70. Therefore, the detection of the high upregulation of the HSP25 protein is a new observation and suggests a prominent role for this small HSP in the membrane regulated stress response. Altogether, we were able to show with these experiments that the membrane fluidizer BA provoked a distinct membrane rearrangements and stress protein response at the growth temperature, manifested by the elevated expression levels of two major HSPs (Fig. 3.). The observed membrane rearrangements (Fig. 2.) are most likely early steps of a signal transduction mechanism aiming to alleviate the effects of stresses on the membrane as it was observed with conditions as cell stretching, shear or osmotic stresses $29,228,233$. It is noteworthy that previous studies have also observed the accumulation of raft-forming lipids as cholesterol, ceramide and saturated phosphoglyceride species both upon heat and BA treatments in B16F10 cells which may contribute to the condensation of plasma membrane domains observed in our studies ${ }^{230}$. Thus according to this data the fluidizing effect was clearly coupled with the reorganization, increased lateral mobility and coalescence of membrane microdomains. This result emphasizes that the effect of heat stress on the membrane is primarily dependent on its fluidizing and not protein denaturizing effect.

Very intriguingly the data showed in Fig. 6. revealed that the presence of HSP25 had a significant effect on the domain size distribution as the number of smaller domains decreased in response to HSP25 expression and the larger domains has accumulated in parallel. These data indicate that HSP25 can directly affect the distribution of membrane domains. Such an action has not been described for HSP25 so far, but it is very likely that this membrane-tailoring is responsible for the membrane buffering effect we observed in Fig. 5 B., which is further emphasizing the role for HSP25 as membrane associated chaperon. 
This data outline in Fig. 4.is underlying a physiological relevance for the membrane fluidization induced membrane microdomain reorganization and subsequent HSP expression. However, it is noteworthy, that we observed no significant difference between the thermotolerance induced by prior heat or membrane fluidization which is remarkable since BA treatment evoked a weaker HSP response relative to heat priming at the HSP level. It is also worth to note that in other studies, no major differences were found in the levels of major HSPs between the heat sensitive B16 parent line and the heat resistant variants, suggesting that HSPs are not the sole determining factors in the heat resistant phenotype of B16 cells ${ }^{234}$. Thus, the acquired heat tolerance observed in this study should involve other mechanisms, not yet identified. It is also noteworthy, that the acquisition of cellular thermotolerance in BA primed Escherichia coli cells was unrelated to the formation of two major HSPs, GroEL (Hsp60) and DnaK (Hsp70). Instead the remodeling of their membrane lipids appeared to be necessary for the development of bacterial thermotolerance ${ }^{235}$. Other studies, using unsaturated fatty acid auxotroph mutants of yeast, also concluded that stress tolerance is strongly associated with the physico-chemical properties and especially with general membrane lipid order of the membrane ${ }^{236}$.

The experiments of Fig. 7 are suggesting that the effect HSP25 on the membrane was exerted by direct interaction with the membrane and was specific to its microdomain regions. Thus these data further confirm our previous notion that HSP25 alters the fine structure of the membrane and its action is mainly associated with membrane microdomain structures. Furthermore, since the membrane sheets are representing a clean system containing only the plasma membrane it is presumable that HSP25 interacts directly with the membrane through its lipids and/or transmembrane proteins.

In summary, our research revealed a significant role for the fluidization induced HSP25 expression in the maintenance of membrane homeostasis. By applying the artificial membrane fluidizer BA we were able to recapitulate the previously observed elevated expression of HSP70 and show the upregulation of HSP25 at the first time at the protein level. Considering the fact that other small HSP family members were characterized to act as membrane associated chaperones ${ }^{38}$ in various model systems, we concentrated our efforts to decipher if HSP25 could act in a similar manner in mammalian cells. Our investigations have led to the discovery of a so far unknown feature of HSP25, which was 
materializing in its effect to act against the forced fluidization applied in our studies. Therefore HSP25 was acted as a membrane buffering agent and helped to maintain the general structure of the membrane. HSP25 promoted membrane domain clustering as the smaller domains were converted into larger ones purely upon the expression of HSP25. This effect was further substantiated by fluorescent correlation spectroscopy measurements where the elevated confinement values were pointing towards a more compartmentalized membrane structure. Also by using such semi-in vitro systems as the isolated membrane sheets we were able to further narrow down the possible interactions of HSP25 directly to the plasma membrane. Although, the description of the precise nature of this interaction and the identification of specific interacting lipids or proteins is still missing, we are certain that this will be an interesting challenge for further studies. Despite of this, these set of experiments contributed to a deeper understanding of the fine regulation of membrane structure and set out the course for future applications that could help to maintain the integrity of the plasma membrane under stress conditions. 


\subsection{Chemotherapy induced PRL3 expression promotes cancer growth via plasma membrane remodeling and specific alterations of caveolae- associated signaling}

As we have described earlier, the plasma membrane is intensely involved in the biochemical events that define pathological conditions such as cancer. The fact that the membrane is the interface for the interaction between malignant cells and non-malignant cells and their stromal environment is giving an immense importance for the membrane in cancer physiology. Consequently membrane level signaling events are described to promote many steps in tumor progression, from early cancer initiation to metastasis. The membrane effectors directly responsible for the cancerous phenotype are functionally regulated by specific membrane domains by defining their sub-membrane localization and interactions. These regulatory membrane domains are dynamic structures as their phase separation behavior is strongly affected by physical forces, underlying cytoskeletal structures, and the adhesion to other cells or matrixes. These associations might be altered in cancerous cells which could provide a basis for malignancies to occur. Consequently, understanding the biochemistry of the plasma membrane is important in the understanding of cancerous diseases. In the experiments we discuss henceforth, we aimed to gain deeper understanding of the role of the membrane within this context. Initially we approached this by searching for a known oncogene which has its tumorigenic action associated with its localization to the plasma membrane. By looking through a number of candidates we have found an intriguing protein which is bound to the membrane by a post translationally added prenyl group, its enzymatic action is coupled with its membrane localization and described to act as an oncogene in several cancerous cases. More importantly its cancer promoting effect was confirmed to depend on its prenylation and thus its localization to the membrane. This candidate was the PRL3 protein, from the group of phosphatases of regenerating liver (PRL) family of dual specificity phosphatases 130,148. The fact that successful approaches were already undertaken to target PRL3 in cancer therapy ${ }^{104}$ was also a factor for choosing it, in order to understand the so far vaguely described action of this protein.

Hereby, we are the first to describe an intriguing upregulation of PRL3 expression in cancer cells upon exposure to genotoxic chemotherapeutic agents. The fact that elevated PRL3 mRNA levels were already observed upon carcinogen treatment in a number of 
cancer cell lines ${ }^{220}$ suggests that PRL3 upregulation upon chemotherapy could also occur in a wide range of cancers. Our results could also be important in explaining previous observations that doxorubicin was more effective in PRL3-silenced leukemia cells ${ }^{237}$, and the results of a drug screen showing that a chemical inhibitor of PRL3 strengthened the antitumor effect of cisplatin ${ }^{121}$. According to our data, this phenomenon might rely on the ability of these drugs to influence the expression of PRL3 in cancer cells. Considering that antibody-based inhibition of PRL3 successfully inhibited tumor reoccurrence after surgical tumor removal in mice ${ }^{104}$, the observed expression of PRL3 upon chemotherapy can also be of critical importance in the re-establishment of cancers. Our in vivo tumor growth data supports this hypothesis, as PRL3-expressing cells were able to establish larger tumors when injected subcutaneously into mice. The connection between PRL3 and tumor initiation was further reinforced by a study describing the significantly lower occurrence of tumors upon carcinogen treatment in PRL3 knockout mice compared to wild type controls ${ }^{123}$.

The PRL3 responsiveness of a cancer cell could rely on a unique genetic background acquired by the cells during oncogenesis. Previously, excessive PRL3 expression was associated with cell cycle stop in a noncancerous cells ${ }^{128}$. Therefore, it is plausible that, within normal conditions, upstream regulators are present in the cells, which could be responsible to keep the effects of PRL3 at bay. These counterbalances might be lost during tumor development. The fact that PRL3 was particularly associated with the initiation of colorectal tumors upon a harsh carcinogenic treatment further suggests that PRL3 might be specifically present in tumors with large-scale genomic mutations ${ }^{123,124}$. As PRL3 was previously detected as a P53 target gene ${ }^{128}$ and there is a detectable level of P53 in all three B16 cells ${ }^{238}$, it is plausible that this transcription factor is responsible for the observed PRL3 induction in our studies. However, as the promoter of PRL3 was described to contain other putative binding elements for stress-related transcription factors, such as NF- $\mathrm{KB}$ and STAT3 ${ }^{239}$, the exact mechanism responsible for PRL3 expression upon stress in cancer cells requires a deeper clarification by future studies.

Our observations also suggest that PRL3 can act in a similar way as phosphatase PP2A, which has been described to regulate the cell cycle through the dephosphorylation of integrin $\beta 1$ on Thr788/789 ${ }^{172}$. As the phosphorylation/ dephosphorylation cycles of the Thr788/789 site was shown to be necessary for the progress through the cell cycle ${ }^{172}$ it is tempting to speculate that this site serves as a switch for regulating adhesion dependent 
cell growth. By modulating this phosphorylation site phosphatases as PRL3 can serve as fast acting switches on integrin receptors and subsequently regulate integrin associated signaling. The observed dual effect of PP2A mediated integrin dephosphorylation, namely, enhanced progression through the cell cycle but decreased cellular adhesion is drawing a strikingly similar picture to what we have observed with PRL3 in the B16F0 cells.

Integrins are well known to translate the forces of mechanical environment of a cell into intracellular signals. In order to do that integrins can recruit further effectors by which they can regulate cellular phenotypes affecting cell cycle progression. Rac1 was shown to be a central mediator in these processes however, the exact mechanism of its membrane recruitment and the specific guanine nucleotide exchange factors (GEFs) that could regulate its activation has not been fully understood yet ${ }^{240}$. Based on the data obtained from our studies we suggest that the caveolar membrane compartment can serve as a primary location for the transmission of mechanical signals by integrins through Rac1 in the context of cell growth regulation. The fact that caveolae and its resident proteins are central to cellular mechanosensation 28 further strengthens the argument for this proposition. Rac1 could control many downstream signaling processes depending on its interaction with regulatory elements. In Rac1 mediated cell adhesion and migration, Rac1 recruitment to the focal adhesion points of the membrane is initiated by the membrane adaptor p130C in concert with integrin $\beta 3$ which allows the interaction between Rac1 and a GEF, Dock180 ${ }^{241}$. However, Dock180 mediated control over Rac1 signaling is associated only with cell adhesion and it is not known what GEFs regulate Rac1 mediated cell cycle signaling. It is plausible that the dephosphorylation of integrin $\beta 1$ at Thr788/789 by PP2A or by PRL3 could act as a recruiting signal towards Rac1. A specific, as yet not known GEF could serve a function in the caveolar compartment for cell cycle regulation. The specific fractionation of Rac1 into the caveolae could define its signaling outcome by spatially restricting its sub membrane localization and subsequently defining its interaction with the caveolae associated regulatory factors and not allowing Rac1 to play a role in other processes as migration and adhesion.

A very intriguing connection was also revealed in regard with the phosphorylation status of integrin $\beta 1$ as it showed a higher degree of indirect actin interaction when it was in a dephosphorylated state at Thr788/789 ${ }^{172}$. This observation could be key to understand our data regarding the increase in the plasma membrane lipid order and the simultaneous activation of lipid raft resident signaling upon PRL3 mediated integrin 
dephosphorylation. Integrin-actin interaction might result in an increased membrane order, since protein complexes consisting of an extracellular anchor (like an integrin) linked to the actin cytoskeleton exert an ordering effect on the membrane ${ }^{242}$. Membrane order and the assembly of membrane microdomains are strongly associated with integrins and subsequently can have major effects on cellular growth ${ }^{34,189}$. Adhesional forces, which define mitotic spindle orientation and subsequent daughter cell retraction, are translated through integrins localized to caveolae domains ${ }^{34}$. In non-adherent conditions the membrane acquires a more symmetric structure which results in a more even localization or endocytosis of the integrin containing membrane domains 32,243. Attachment and detachment alters membrane order also in an integrin and caveolin associated manner ${ }^{189}$. The altered cellular geometry and increased membrane fluidity upon detachment or upon the natural cell rounding during the mitotic phase might be responsible for the inherent growth inhibition activated in non-adherent cells ${ }^{32,73}$. It is possible that in altered rounded conditions the more fluid membrane does not support the concentrated localization of the domains especially in the lack of a guiding integrin signal. By altering the membrane order possibly through the regulation of actin-integrin interaction, the cell might gain an ability to push faster through the growth retaining mechanism associated with the lack of adherence or with the natural mitotic cell rounding during the cell cycle. This assumption is in line with the hypothesis that the dephosphorylation of integrin $\beta$ at Thr788/789 by PP2A is helping the membrane to strengthen its connection with the cortical actin filaments in order to aid the reentering of the cell to the $\mathrm{G} 1$ phase from the G2/M phase ${ }^{172}$.

It is noteworthy that the amount of caveolae in the membrane decreases during cancer progression ${ }^{19,244}$, therefore caveolae might be present in early-stage cancers but not in more advanced tumors. The reason why PRL3 is connected to such a wide variety of signaling pathways in different cancer cells ${ }^{130}$ might rely on the fact that PRL3 is not in its original subcellular environment in those tumors where caveolae are absent. Therefore, considering the wide, possible substrate variety of PRL3, nonspecific interactions could account for the diverse actions observed for PRL3. The importance of caveolae in downstream PRL3 signaling could be highlighted by comparing PRL3 effect on Rho GTPases in different cell lines. In cells lacking caveolin ${ }^{245}$, PRL3 decreased Rac1 GTP-binding ${ }^{113,114}$ and increased the activity of other Rho GTPases. However, in other cell lines where caveolin expression was detectable ${ }^{246}$, PRL3 expression was associated with enhanced GTP-loading of Rac1 or increased Rac1 protein levels ${ }^{162,247}$. 
The hypothesis that PRL3 exerts a different role depending on the existence of caveolar structures is further strengthened by the observation that in primary uveal melanoma cells PRL3 affects integrin clustering predominantly at the focal adhesion sites of the membrane ${ }^{151}$. Uveal melanoma cells, however, express a considerably lower, or no amounts of Caveolin 1 compared to melanoma cells of cutaneous origins ${ }^{248}$, such as B16F0 cells.

Moreover, the structural membrane changes that we observed during PRL3 expression might contribute to the step-by-step progression of cancer cells towards a more aggressive phenotype. Stress conditions, such as mechanical stretching or osmotic swelling, are associated with the disassembly of caveolae ${ }^{28}$. Therefore, it is tempting to speculate that the increased membrane rigidity in the PRL3-expressing cells might result in a similar cellular response in the long term. This hypothesis is substantiated by our morphological observations describing elongated, unnatural caveolae structures in the PRL3-expressing cells. Such a loss of caveolae from early-stage cancer cells could consequentially contribute to cancer progression ${ }^{245}$. Membrane raft reorganization and membrane fluidity alterations were already connected with gene expression changes and stress resistance in B16 melanoma cells ${ }^{232}$.

In summary, we identified PRL3 as a protein upregulated in cancer cells upon treatment with several clinically relevant antitumor therapeutics. In our melanoma model, PRL3 expression was strongly associated with enhanced cancer growth in parallel with increased cyclin D1 levels. The elevation of cyclin D1 was shown to be associated with the dephosphorylation of integrin $\beta 1$ at Thr788/789 by PRL3 and the subsequent attraction of Rac1 to the plasma membrane. Therefore, here we propose that the genotoxic stress-mediated induction of PRL3 could rewire caveolae signaling, which can be critical in tumor initiation and relapse. In addition, our study also highlights the importance of plasma membrane organization in cancer pathology and therapy. In conclusion, targeting PRL3 in parallel with chemotherapy might prove useful for the therapy of PRL3-expressing cancers. 


\section{Summary of findings}

The most important findings obtained during the current thesis work are summarized as follows:

- On the level of membrane regulated stress signaling and adaptation:

○ By applying the artificial membrane fluidizer BA we were able to recapitulate the previously observed elevated expression of HSP70 and showed the upregulation of HSP25 for the first time at the protein level.

- Our investigations have led to the discovery of a so far unknown feature of HSP25, which was materializing in its effect to act against the forced membrane fluidization applied in our studies. Which was presumably taking part in the acquisition of a stress resistant phenotype in BA preconditioned cells.

- HSP25 exerted its effect on the membrane by promoting membrane domain clustering as the smaller domains coalesced into larger ones purely upon the expression of HSP25.

- This effect was further substantiated by fluorescent correlation spectroscopy measurements where the elevated confinement values pointed towards a more compartmentalized membrane structure. By using such a "semi-in vitro" system as the isolated membrane sheets we were able to further narrow down the possible interactions of HSP25 to membrane lipids and transmembrane proteins.

- On the level of membrane associated oncogenesis:

O Here we identified PRL3 as a protein upregulated in cancer cells, in a time and dose dependent manner upon treatments with several clinically relevant anti-tumor therapeutics.

- Our experiments were able to successfully connect the presence of PRL3 with a distinct phenotype that could promote tumor progression by affecting tumor growth.

- PRL3 was shown to affect the structure of the membrane and associate with the caveolae type lipid raft structures of the plasma membrane.

- PRL3 was associated with the caveolae proteome as it was detected to dephosphorylate integrin $\beta 1$ at its Thr788/789 site.

- PRL3 expression was also associated with increased plasma membrane caveolae region targeting and subsequent activation of Rac1.

- Rac1 was affiliated with the activation of the following signaling pathway: Rac1-GTP/AKT/GSK3 $\beta$ which led to the nuclear accumulation of the cyclin D1 molecule. 


\section{References}

1. Alberts, Johnson, Lewis, Raff, Roberts \& Walter. Molecular biology of the cell. (Garland Science, 2002).

2. Simons \& Sampaio. Membrane organization and lipid rafts. Cold Spring Harb. Perspect. Biol. 3, a004697 (2011).

3. Shevchenko \& Simons. Lipidomics: coming to grips with lipid diversity. Nat. Rev. Mol. Cell Biol. 11, 593-598 (2010).

4. Futerman \& Hannun. The complex life of simple sphingolipids. EMBO Rep. 5, 777-82 (2004).

5. Singer \& Nicolson. The Fluid Mosaic Model of the Structure of Cell Membranes. Science (80-. ). 175, (1972).

6. van den Brink-van der Laan, Antoinette Killian \& de Kruijff. Nonbilayer lipids affect peripheral and integral membrane proteins via changes in the lateral pressure profile. Biochim. Biophys. Acta - Biomembr. 1666, 275-288 (2004).

7. Daleke. Regulation of transbilayer plasma membrane phospholipid asymmetry. $J$. Lipid Res. 44, 233-42 (2003).

8. Contreras, Ernst, Wieland \& Brugger. Specificity of Intramembrane Protein-Lipid Interactions. Cold Spring Harb. Perspect. Biol. 3, a004705-a004705 (2011).

9. Vereb, Szollosi, Matko, Nagy, Farkas, Vigh, Matyus, Waldmann \& Damjanovich. Dynamic, yet structured: The cell membrane three decades after the SingerNicolson model. Proc. Natl. Acad. Sci. 100, 8053-8058 (2003).

10. Kusumi, Nakada, Ritchie, Murase, Suzuki, Murakoshi, Kasai, Kondo \& Fujiwara. Paradigm Shift of the Plasma Membrane Concept from the Two-Dimensional Continuum Fluid to the Partitioned Fluid: High-Speed Single-Molecule Tracking of Membrane Molecules. Annu. Rev. Biophys. Biomol. Struct. 34, 351-378 (2005).

11. Nicolson. The Fluid-Mosaic Model of Membrane Structure: Still relevant to understanding the structure, function and dynamics of biological membranes after more than 40years. Biochim. Biophys. Acta - Biomembr. 1838, 1451-1466 (2014).

12. Israelachvili, Marcelja \& Horn. Physical principles of membrane organization. $Q$. Rev. Biophys. 13, 121-200 (1980).

13. Karnovsky, Kleinfeld, Hoover \& Klausner. The concept of lipid domains in membranes. J. Cell Biol. 94, 1-6 (1982).

14. Simons \& van Meer. Lipid sorting in epithelial cells. Biochemistry 27, 6197-202 (1988).

15. Simons \& Ikonen. Functional rafts in cell membranes. Nature 387, 569-572 (1997).

16. Pike. The challenge of lipid rafts. J. Lipid Res. 50 Suppl, S323-8 (2009).

17. Pike. Rafts defined: a report on the Keystone Symposium on Lipid Rafts and Cell Function. J. Lipid Res. 47, 1597-8 (2006).

18. Parton \& del Pozo. Caveolae as plasma membrane sensors, protectors and organizers. Nat. Rev. Mol. Cell Biol. 14, 98-112 (2013).

19. Martinez-Outschoorn, Sotgia \& Lisanti. Caveolae and signalling in cancer. Nat. Rev. Cancer 15, 225-237 (2015).

20. Simons \& Ikonen. Functional rafts in cell membranes. Nature 387, 569-72 (1997). 
21. Giocondi, Besson, Dosset, Milhiet \& Le Grimellec. Temperature-dependent localization of GPI-anchored intestinal alkaline phosphatase in model rafts. J. Mol. Recognit. 20, 531-7

22. Simons \& Gerl. Revitalizing membrane rafts: new tools and insights. Nat. Rev. Mol. Cell Biol. 11, 688-99 (2010).

23. van Meer. Caveolin, cholesterol, and lipid droplets? J. Cell Biol. 152, F29-34 (2001).

24. Gutierrez-Pajares, Iturrieta, Dulam, Wang, Pavlides, Malacari, Lisanti \& Frank. Caveolin-3 Promotes a Vascular Smooth Muscle Contractile Phenotype. Front. Cardiovasc. Med. 2, 27 (2015).

25. Briand, Dugail \& Le Lay. Cavin proteins: New players in the caveolae field. Biochimie 93, 71-77 (2011).

26. Nabi. Cavin fever: regulating caveolae. Nat. Cell Biol. 11, 789-791 (2009).

27. Parton \& del Pozo. Caveolae as plasma membrane sensors, protectors and organizers. Nat. Rev. Mol. Cell Biol. 14, 98-112 (2013).

28. Sinha, Köster, Ruez, Gonnord, Bastiani, Abankwa, Stan, Butler-Browne, Vedie, Johannes, Morone, Parton, Raposo, Sens, Lamaze \& Nassoy. Cells respond to mechanical stress by rapid disassembly of caveolae. Cell 144, 402-13 (2011).

29. Joshi, Bastiani, Strugnell, Boscher, Parton \& Nabi. Phosphocaveolin-1 is a mechanotransducer that induces caveola biogenesis via Egr1 transcriptional regulation. J. Cell Biol. 199, 425-35 (2012).

30. Boucrot, Howes, Kirchhausen \& Parton. Redistribution of caveolae during mitosis. J. Cell Sci. 124, 1965-72 (2011).

31. del Pozo, Alderson, Kiosses, Chiang, Anderson \& Schwartz. Integrins regulate Rac targeting by internalization of membrane domains. Science 303, 839-42 (2004).

32. del Pozo, Balasubramanian, Alderson, Kiosses, Grande-García, Anderson \& Schwartz. Phospho-caveolin-1 mediates integrin-regulated membrane domain internalization. Nat. Cell Biol. 7, 901-8 (2005).

33. Galbiati, Volonté, Liu, Capozza, Frank, Zhu, Pestell \& Lisanti. Caveolin-1 expression negatively regulates cell cycle progression by inducing $\mathrm{G}(0) / \mathrm{G}(1)$ arrest via a p53/p21(WAF1/Cip1)-dependent mechanism. Mol. Biol. Cell 12, 2229-44 (2001).

34. Matsumura, Kojidani, Kamioka, Uchida, Haraguchi, Kimura \& Toyoshima. Interphase adhesion geometry is transmitted to an internal regulator for spindle orientation via caveolin-1. Nat. Commun. 7, ncomms11858 (2016).

35. Baker \& Tuan. The less-often-traveled surface of stem cells: caveolin-1 and caveolae in stem cells, tissue repair and regeneration. Stem Cell Res. Ther. 4, 90 (2013).

36. Kültz. Evolution of the cellular stress proteome: from monophyletic origin to ubiquitous function. J Exp Biol 206, 3119-3124 (2003).

37. Kültz. Molecular and evolutionary basis of the cellular stress response. Annu. Rev. Physiol. 67, 225-57 (2005).

38. Horváth, Multhoff, Sonnleitner \& Vígh. Membrane-associated stress proteins: more than simply chaperones. Biochim. Biophys. Acta 1778, 1653-64

39. Lindquist. The Heat-Shock Response. Annu. Rev. Biochem. 55, 1151-1191 (1986). 
40. Morimoto. Regulation of the heat shock transcriptional response: cross talk between a family of heat shock factors, molecular chaperones, and negative regulators. Genes Dev. 12, 3788-96 (1998).

41. Kampinga. Chaperones in preventing protein denaturation in living cells and protecting against cellular stress. Handb. Exp. Pharmacol. 1-42 (2006).

42. Van Montfort, Slingsby \& Vierling. Structure and function of the small heat shock protein/alpha-crystallin family of molecular chaperones. Adv. Protein Chem. 59, 105-56 (2001).

43. Andley. Crystallins in the eye: Function and pathology. Prog. Retin. Eye Res. 26, 78-98 (2007).

44. Tang. Critical role of actin-associated proteins in smooth muscle contraction, cell proliferation, airway hyperresponsiveness and airway remodeling. Respir. Res. 16, 134 (2015).

45. Koll, Guiard, Rassow, Ostermann, Horwich, Neupert \& Hartl. Antifolding activity of hsp60 couples protein import into the mitochondrial matrix with export to the intermembrane space. Cell 68, 1163-75 (1992).

46. Mayer \& Bukau. Hsp70 chaperones: Cellular functions and molecular mechanism. Cell. Mol. Life Sci. 62, 670-684 (2005).

47. Brown, Zhu, Schmidt \& Tucker. Hsp90--from signal transduction to cell transformation. Biochem. Biophys. Res. Commun. 363, 241-6 (2007).

48. Schirmer, Glover, Singer \& Lindquist. HSP100/Clp proteins: a common mechanism explains diverse functions. Trends Biochem. Sci. 21, 289-96 (1996).

49. Horváth, Glatz, Varvasovszki, Török, Páli, Balogh, Kovács, Nádasdi, Benkö, Joó \& Vígh. Membrane physical state controls the signaling mechanism of the heat shock response in Synechocystis PCC 6803: identification of hsp17 as a 'fluidity gene'. Proc. Natl. Acad. Sci. U. S. A. 95, 3513-8 (1998).

50. Horváth, Glatz, Nakamoto, Mishkind, Munnik, Saidi, Goloubinoff, Harwood \& Vigh. Heat shock response in photosynthetic organisms: membrane and lipid connections. Prog. Lipid Res. 51, 208-20 (2012).

51. Vigh, Horváth, Maresca \& Harwood. Can the stress protein response be controlled by 'membrane-lipid therapy'? Trends Biochem Sci 32, 357-363 (2007).

52. Vigh, Los, Horváth \& Murata. The primary signal in the biological perception of temperature: Pd-catalyzed hydrogenation of membrane lipids stimulated the expression of the desA gene in Synechocystis PCC6803. Proc. Natl. Acad. Sci. U. S. A. 90, 9090-4 (1993).

53. Balogh, Horváth, Nagy, Hoyk, Benkõ, Bensaude \& Vígh. The hyperfluidization of mammalian cell membranes acts as a signal to initiate the heat shock protein response. FEBS J. 272, 6077-86 (2005).

54. Balogh, Péter, Glatz, Gombos, Török, Horváth, Harwood \& Vígh. Key role of lipids in heat stress management. FEBS Lett. (2013).

55. Horváth, Török, Vígh \& Kates. Lipid hydrogenation induces elevated 18:1-CoA desaturase activity in Candida lipolytica microsomes. Biochim Biophys Acta 1085, 126-130 (1991).

56. Török, Crul, Maresca, Schütz, Viana, Dindia, Piotto, Brameshuber, Balogh, Péter, Porta, Trapani, Gombos, Glatz, Gungor, Peksel, Vigh, Csoboz, Horváth, Vijayan, Hooper \& Harwood. Plasma membranes as heat stress sensors: From lipid- 
controlled molecular switches to therapeutic applications. Biochim. Biophys. Acta 1838, 1594-1618 (2014).

57. Glatz, Horváth, Varvasovszki, Kovács, Török \& Vigh. Chaperonin genes of the Synechocystis PCC 6803 are differentially regulated under light-dark transition during heat stress. Biochem Biophys Res Commun 239, 291-297 (1997).

58. Balogi, Cheregi, Giese, Juhász, Vierling, Vass, Vígh \& Horváth. A mutant small heat shock protein with increased thylakoid association provides an elevated resistance against UV-B damage in synechocystis 6803. J Biol Chem 283, 2298322991 (2008).

59. Török, Goloubinoff, Horváth, Tsvetkova, Glatz, Balogh, Varvasovszki, Los, Vierling, Crowe \& Vigh. Synechocystis HSP17 is an amphitropic protein that stabilizes heat-stressed membranes and binds denatured proteins for subsequent chaperone-mediated refolding. Proc. Natl. Acad. Sci. U. S. A. 98, 3098-103 (2001).

60. Tsvetkova, Horváth, Török, Wolkers, Balogi, Shigapova, Crowe, Tablin, Vierling, Crowe \& Vigh. Small heat-shock proteins regulate membrane lipid polymorphism. Proc Natl Acad Sci U S A 99, 13504-13509 (2002).

61. Miyake, Araki \& Tsuchido. Synthesis and Sedimentation of a Subset of 15-kDa Heat Shock Proteins in Escherichia coli Cells Recovering from Sublethal Heat Stress. http://dx.doi.org/10.1271/bbb.57.578 (2014). doi:10.1271/BBB.57.578

62. Coucheney, Gal, Beney, Lherminier, Gervais \& Guzzo. A small HSP, Lo18, interacts with the cell membrane and modulates lipid physical state under heat shock conditions in a lactic acid bacterium. Biochim. Biophys. Acta-Biomembr. 1720, 92-98 (2005).

63. Lee, Hefta \& Brennan. Characterization of the major membrane protein of virulent Mycobacterium tuberculosis. Infect. Immun. 60, 2066-74 (1992).

64. Nakagawa, Tsujimoto, Nakagawa, Iwaki, Fukumaki \& Iwaki. Association of HSPB2, a Member of the Small Heat Shock Protein Family, with Mitochondria. Exp. Cell Res. 271, 161-168 (2001).

65. Cobb \& Petrash. Characterization of alpha-crystallin-plasma membrane binding. $J$. Biol. Chem. 275, 6664-72 (2000).

66. Bellyei, Szigeti, Pozsgai, Boronkai, Gomori, Hocsak, Farkas, Sumegi \& Gallyas. Preventing apoptotic cell death by a novel small heat shock protein. Eur. J. Cell Biol. 86, 161-171 (2007).

67. Bellyei, Szigeti, Boronkai, Pozsgai, Gomori, Melegh, Janaky, Bognar, Hocsak, Sumegi \& Gallyas. Inhibition of cell death by a novel $16.2 \mathrm{kD}$ heat shock protein predominantly via Hsp90 mediated lipid rafts stabilization and Akt activation pathway. Apoptosis 12, 97-112 (2007).

68. Tsvetkova, Horvath, Torok, Wolkers, Balogi, Shigapova, Crowe, Tablin, Vierling, Crowe \& Vigh. Small heat-shock proteins regulate membrane lipid polymorphism. Proc. Natl. Acad. Sci. 99, 13504-13509 (2002).

69. Pavlides, Gutierrez-Pajares, Iturrieta, Lisanti \& Frank. Endothelial caveolin-1 plays a major role in the development of atherosclerosis. Cell Tissue Res. 356, 147-157 (2014).

70. Bruno, Sotgia, Gazzerro, Minetti \& Lisanti. Caveolinopathies. GeneReviews( $\left.{ }^{\circledR}\right)$ (1993). 
71. Jasmin, Rengo, Lymperopoulos, Gupta, Eaton, Quann, Gonzales, Mercier, Koch \& Lisanti. Caveolin-1 deficiency exacerbates cardiac dysfunction and reduces survival in mice with myocardial infarction. AJP Hear. Circ. Physiol. 300, H1274H1281 (2011).

72. Echarri, Muriel \& Del Pozo. Intracellular trafficking of raft/caveolae domains: insights from integrin signaling. Semin. Cell Dev. Biol. 18, 627-37 (2007).

73. Cerezo, Guadamillas, Goetz, Sánchez-Perales, Klein, Assoian \& del Pozo. The absence of caveolin-1 increases proliferation and anchorage- independent growth by a Rac-dependent, Erk-independent mechanism. Mol. Cell. Biol. 29, 5046-59 (2009).

74. Gombos, Crul, Piotto, Güngör, Török, Balogh, Péter, Slotte, Campana, Pilbat, Hunya, Tóth, Literati-Nagy, Vígh, Glatz, Brameshuber, Schütz, Hevener, Febbraio, Horváth \& Vígh. Membrane-lipid therapy in operation: the HSP coinducer BGP-15 activates stress signal transduction pathways by remodeling plasma membrane rafts. PLoS One 6, e28818 (2011).

75. Llado, Gutierrez, Martínez, Casas, Terés, Higuera, Galmés, Saus, Besalduch, Busquets \& Escribá. MINERVAL INDUCES APOPTOSIS IN JURKAT AND OTHER CANCER CELLS. J. Cell. Mol. Med. 14, 659-70 (2008).

76. Busto, Del Canto-Jañez, Goñi, Mollinedo \& Alonso. Combination of the antitumour cell ether lipid edelfosine with sterols abolishes haemolytic side effects of the drug. J. Chem. Biol. 1, 89-94 (2008).

77. Diamond, Cressman, Laz, Abrams \& Taub. PRL-1, a unique nuclear protein tyrosine phosphatase, affects cell growth. Mol. Cell. Biol. 14, 3752-62 (1994).

78. Zeng, Hong \& Tan. Mouse PRL-2 and PRL-3, Two Potentially Prenylated Protein Tyrosine Phosphatases Homologous to PRL-1. Biochem. Biophys. Res. Commun. 244, 421-427 (1998).

79. Zeng, Dong, Guo, Li, Tan, Koh, Pallen, Manser \& Hong. PRL-3 and PRL-1 promote cell migration, invasion, and metastasis. Cancer Res. 63, 2716-22 (2003).

80. Stephens, Han, Gokhale \& Von Hoff. PRL phosphatases as potential molecular targets in cancer. Mol. Cancer Ther. 4, 1653-1661 (2005).

81. Kozlov. Structural Insights into Molecular Function of the Metastasis-associated Phosphatase PRL-3. J. Biol. Chem. 279, 11882-11889 (2004).

82. Kim, Song, Jee, Sheen, Lee, Lee, Ro, Cho, Lee, Yamazaki, Jeon \& Cheong. Structure of human PRL-3, the phosphatase associated with cancer metastasis. FEBS Lett. 565, 181-187 (2004).

83. Ishii, Funato \& Miki. Thioredoxin-related protein 32 (TRP32) specifically reduces oxidized phosphatase of regenerating liver (PRL). J. Biol. Chem. 288, 7263-70 (2013).

84. Di Cara, Maile, Parsons, Magico, Basu, Tapon \& King-Jones. The Hippo pathway promotes cell survival in response to chemical stress. Cell Death Differ. 22, 152639 (2015).

85. Sun, Luo, Yu, Wang, Zhou, Liang \& Zhang. Phosphatase Activity, Trimerization, and the C-terminal Polybasic Region Are All Required for PRL1-mediated Cell Growth and Migration. J. Biol. Chem. 282, 29043-29051 (2007).

86. Tonks. Protein tyrosine phosphatases: from genes, to function, to disease. Nat. Rev. Mol. Cell Biol. 7, 833-846 (2006). 
87. Wang \& Casey. Protein prenylation: unique fats make their mark on biology. Nat. Rev. Mol. Cell Biol. 17, 110-122 (2016).

88. Zeng, Si, Horstmann, Xu, Hong \& Pallen. Prenylation-dependent association of protein-tyrosine phosphatases PRL-1, -2 , and -3 with the plasma membrane and the early endosome. J. Biol. Chem. 275, 21444-52 (2000).

89. Pascaru, Tanase, Vacaru, Boeti, Neagu, Popescu \& Szedlacsek. Analysis of molecular determinants of PRL-3. J. Cell. Mol. Med. 13, 3141-3150 (2009).

90. Chavan, Muratcioglu, Marszalek, Jang, Keskin, Gursoy, Nussinov \& Gaponenko. Plasma membrane regulates Ras signaling networks. Cell. Logist. 5, e1136374 (2015).

91. Saha, Bardelli, Buckhaults, Velculescu, Rago, St Croix, Romans, Choti, Lengauer, Kinzler \& Vogelstein. A phosphatase associated with metastasis of colorectal cancer. Science 294, 1343-6 (2001).

92. Bessette, Qiu \& Pallen. PRL PTPs: mediators and markers of cancer progression. Cancer Metastasis Rev. 27, 231-52 (2008).

93. Polato, Codegoni, Fruscio, Perego, Mangioni, Saha, Bardelli \& Broggini. PRL-3 phosphatase is implicated in ovarian cancer growth. Clin. Cancer Res. 11, 6835-9 (2005).

94. Wang, Cai, He, Zhan, Chen, Cui, Wu, Wu, Song, Zhang, Peng \& Huang. High expression of PRL-3 can promote growth of gastric cancer and exhibits a poor prognostic impact on patients. Ann. Surg. Oncol. 16, 208-19 (2009).

95. Bilici, Ustaalioglu, Yavuzer, Seker, Mayadagli \& Gumus. Prognostic significance of high phosphatase of regenerating liver-3 expression in patients with gastric cancer who underwent curative gastrectomy. Dig. Dis. Sci. 57, 1568-75 (2012).

96. Dai, Lu, Shou \& Li. Expression of phosphatase regenerating liver 3 is an independent prognostic indicator for gastric cancer. World J. Gastroenterol. 15, 1499-505 (2009).

97. Hao, Zhang, Pan, Liu, Xiang, Wan \& Wu. Prognostic and metastatic value of phosphatase of regenerating liver-3 in invasive breast cancer. J. Cancer Res. Clin. Oncol. 136, 1349-57 (2010).

98. Wang, Peng, Dong, Kong, Meng, Yan, Xie \& Shou. Overexpression of phosphatase of regenerating liver-3 in breast cancer: association with a poor clinical outcome. Ann. Oncol. 17, 1517-22 (2006).

99. Mayinuer, Yasen, Mogushi, Obulhasim, Xieraili, Aihara, Tanaka, Mizushima, Tanaka \& Arii. Upregulation of protein tyrosine phosphatase type IVA member 3 (PTP4A3/PRL-3) is associated with tumor differentiation and a poor prognosis in human hepatocellular carcinoma. Ann. Surg. Oncol. 20, 305-17 (2013).

100. Ren, Jiang, Xing, Dong, Peng, Meng, Xu \& Shou. Prognostic significance of phosphatase of regenerating liver-3 expression in ovarian cancer. Pathol. Oncol. Res. 15, 555-60 (2009).

101. Xing, Peng, Qu, Ren, Dong, Su \& Shou. Prognostic value of PRL-3 overexpression in early stages of colonic cancer. Histopathology 54, 309-18 (2009).

102. Kato, Semba, Miskad, Seo, Kasuga \& Yokozaki. High expression of PRL-3 promotes cancer cell motility and liver metastasis in human colorectal cancer: a predictive molecular marker of metachronous liver and lung metastases. Clin. 
Cancer Res. 10, 7318-28 (2004).

103. Tamagawa, Oshima, Yoshihara, Watanabe, Numata, Yamamoto, Tuschida, Shiozawa, Morinaga, Akaike, Masuda \& lmada. The expression of the phosphatase regenerating liver 3 gene is associated with outcome in patients with colorectal cancer. Hepatogastroenterology. 59, 2122-6 (2012).

104. Thura, Al-Aidaroos, Yong, Kono, Gupta, Lin, Mimura, Thiery, Goh, Tan, Soo, Hong, Wang, Lin, Chen, Rha, Chung, Li, Nandi, Yuen, Zhang, Guan, So \& Zeng. PRL3-zumab, a first-in-class humanized antibody for cancer therapy. JCI Insight 1, (2016).

105. Guo, Li, Tang, Koh, Gan \& Zeng. Catalytic domain of PRL-3 plays an essential role in tumor metastasis: formation of PRL-3 tumors inside the blood vessels. Cancer Biol. Ther. 3, 945-51 (2004).

106. Guo, Li, Wang, Osato, Tang, Quah, Gan \& Zeng. PRL-3 Initiates Tumor Angiogenesis by Recruiting Endothelial Cells In vitro and In vivo. Cancer Res. 66, 9625-9635 (2006).

107. Ming, Liu, Gu, Qiu \& Wang. PRL-3 facilitates angiogenesis and metastasis by increasing ERK phosphorylation and up-regulating the levels and activities of Rho-A/C in lung cancer. Pathology 41, 118-126 (2009).

108. Zeng, Dong, Guo, Li, Tan, Koh, Pallen, Manser \& Hong. PRL-3 and PRL-1 Promote Cell Migration, Invasion, and Metastasis. Cancer Res. 63, (2003).

109. Bessette, Wong \& Pallen. PRL-3: A Metastasis-Associated Phosphatase in Search of a Function. Cells Tissues Organs 185, 232-236 (2007).

110. Peng, Xing, Li, Qu, Meng, Lian, Jiang, Wu \& Shou. PRL-3 promotes the motility, invasion, and metastasis of LoVo colon cancer cells through PRL-3-integrin beta1ERK1/2 and-MMP2 signaling. Mol. Cancer 8, 110 (2009).

111. Forte, Orsatti, Talamo, Barbato, De Francesco \& Tomei. Ezrin is a specific and direct target of protein tyrosine phosphatase PRL-3. Biochim. Biophys. Acta-Mol. Cell Res. 1783, 334-344 (2008).

112. McClatchey. Opinion: Merlin and ERM proteins: unappreciated roles in cancer development? Nat. Rev. Cancer 3, 877-883 (2003).

113. Fiordalisi, Keller \& Cox. PRL Tyrosine Phosphatases Regulate Rho Family GTPases to Promote Invasion and Motility. Cancer Res. 66, 3153-3161 (2006).

114. Wang, Quah, Dong, Manser, Tang \& Zeng. PRL-3 Down-regulates PTEN Expression and Signals through PI3K to Promote Epithelial-Mesenchymal Transition. Cancer Res. 67, 2922-2926 (2007).

115. Liang, Liang, Wang, Sun, Udho \& Zhang. PRL3 promotes cell invasion and proliferation by down-regulation of Csk leading to Src activation. J. Biol. Chem. 282, 5413-9 (2007).

116. Polato, Codegoni, Fruscio, Perego, Mangioni, Saha, Bardelli \& Broggini. PRL-3 Phosphatase Is Implicated in Ovarian Cancer Growth. Clin. Cancer Res. 11, (2005).

117. Daouti, Li, Qian, Huang, Holmgren, Levin, Reik, McGady, Gillespie, Perrotta, Bian, Reidhaar-Olson, Bliss, Olivier, Sergi, Fry, Danho, Ritland, Fotouhi, Heimbrook \& Niu. A selective phosphatase of regenerating liver phosphatase inhibitor suppresses tumor cell anchorage-independent growth by a novel mechanism involving p130Cas cleavage. Cancer Res. 68, 1162-9 (2008). 
118. Ye, Al-aidaroos, Park, Yuen, Zhang, Gupta, Lin, Shen \& Zeng. PRL-3 activates mTORC1 in Cancer Progression. Sci. Rep. 5, 17046 (2015).

119. Al-Aidaroos, Yuen, Guo, Zhang, Chung, Chng \& Zeng. Metastasis-associated PRL-3 induces EGFR activation and addiction in cancer cells. J. Clin. Invest. 123, 3459-71 (2013).

120. Jiang, Liu, Rajput, Geng, Ongchin, Zeng, Taylor \& Wang. Phosphatase PRL-3 is a direct regulatory target of TGFbeta in colon cancer metastasis. Cancer Res. 71, 234-44 (2011).

121. Xia, Yang, Li, Li, Zhou, Dai \& Wong. Image-based chemical screening identifies drug efflux inhibitors in lung cancer cells. Cancer Res. 70, 7723-33 (2010).

122. Qu, Liu, Guo, Shi, Zhou, Li, Yang, Tong \& Wang. Independent oncogenic and therapeutic significance of phosphatase PRL-3 in FLT3-ITD-negative acute myeloid leukemia. Cancer 120, 2130-41 (2014).

123. Zimmerman, Homanics \& Lazo. Targeted deletion of the metastasis-associated phosphatase Ptp4a3 (PRL-3) suppresses murine colon cancer. PLoS One 8, e58300 (2013).

124. Cramer, Zimmerman, Thompson, Homanics, Lazo \& Lagasse. Deletion of Ptp4a3 reduces clonogenicity and tumor-initiation ability of colitis-associated cancer cells in mice. Stem Cell Res. 13, 164-71 (2014).

125. Zhou, Chan, Bi, Lu, Chong, Chooi, Cheong, Liu, Ching, Zhou, Osato, Tan, Ng, $\mathrm{Ng}$, Wang, Zeng \& Chng. LIN28B Activation by PRL-3 Promotes Leukemogenesis and a Stem Cell-like Transcriptional Program in AML. Mol. Cancer Res. 15, (2017).

126. Kobayashi, Bai, Dong, Yu, Chen, Gao, Zhang, Yoder, Kapur, Zhang \& Liu. PRL2/PTP4A2 phosphatase is important for hematopoietic stem cell self-renewal. Stem Cells 32, 1956-67 (2014).

127. Dong, Zhang, Bai, Zhou, Campbell, Chen, Yong, Zhang, Zeng, Shou \& Zhang. Phosphatase of regenerating liver 2 (PRL2) deficiency impairs Kit signaling and spermatogenesis. J. Biol. Chem. 289, 3799-810 (2014).

128. Basak, Jacobs, Krieg, Pathak, Zeng, Kaldis, Giaccia \& Attardi. The metastasisassociated gene Prl-3 is a p53 target involved in cell-cycle regulation. Mol. Cell 30, 303-14 (2008).

129. Al-Aidaroos \& Zeng. PRL-3 phosphatase and cancer metastasis. J. Cell. Biochem. 111, 1087-1098 (2010).

130. Rios, Li \& Köhn. Molecular mechanisms of the PRL phosphatases. FEBS J. 280, 505-24 (2013).

131. Rubio \& Köhn. Regulatory mechanisms of phosphatase of regenerating liver (PRL)-3. Biochem. Soc. Trans. 44, 1305-1312 (2016).

132. $\mathrm{Xu}, \mathrm{Cao}, \mathrm{Wang}, \mathrm{Xu}, \mathrm{Chen} \& \mathrm{Xu}$. VEGF Promotes the Transcription of the Human PRL-3 Gene in HUVEC through Transcription Factor MEF2C. PLoS One 6, e27165 (2011).

133. Lian, Meng, Liu, Xing, Song, Dong, Han, Yang, Peng, Qu \& Shou. PRL-3 activates NF- $\kappa \mathrm{B}$ signaling pathway by interacting with RAP1. Biochem. Biophys. Res. Commun. 430, 196-201 (2013).

134. Wang, Quah, Dong, Manser, Tang \& Zeng. PRL-3 down-regulates PTEN expression and signals through PI3K to promote epithelial-mesenchymal 
transition. Cancer Res. 67, 2922-6 (2007).

135. Slørdahl, Abdollahi, Vandsemb, Rampa, Misund, Baranowska, Westhrin, Waage, $\mathrm{R} \varnothing \& \mathrm{~B} \emptyset \mathrm{rset}$. The phosphatase of regenerating liver-3 (PRL-3) is important for IL6-mediated survival of myeloma cells. Oncotarget 7, 27295-306 (2016).

136. Park, Yuen, Zhou, Al-Aidaroos, Guo, Valk, Zhang, Chng, Hong, Mills \& Zeng. Oncogenic roles of PRL-3 in FLT3-ITD induced acute myeloid leukaemia. EMBO Mol. Med. 5, 1351-66 (2013).

137. Min, Kim, Heo, Kim, Kim \& Yoo. Downregulation of p53 by phosphatase of regenerating liver 3 is mediated by MDM2 and PIRH2. Life Sci. 86, 66-72 (2010).

138. Zheng, Meng, Gao, Chen, Liu, Xiao, Liu, Sui, Zhou, Liu \& Li. Snail as a key regulator of PRL-3 gene in colorectal cancer. Cancer Biol. Ther. 12, 742-749 (2011).

139. Villarejo, Cortés-Cabrera, Molina-Ortíz, Portillo \& Cano. Differential role of Snail1 and Snail2 zinc fingers in E-cadherin repression and epithelial to mesenchymal transition. J. Biol. Chem. 289, 930-41 (2014).

140. Wang, Vardy, Tan, Loo, Guo, Li, Lim, Zhou, Chng, Ng, Li \& Zeng. PCBP1 Suppresses the Translation of Metastasis-Associated PRL-3 Phosphatase. Cancer Cell 18, 52-62 (2010).

141. Fiordalisi, Dewar, Graves, Madigan \& Cox. Src-mediated phosphorylation of the tyrosine phosphatase PRL-3 is required for PRL-3 promotion of Rho activation, motility and invasion. PLoS One 8, e64309 (2013).

142. Choi, Min, Jung, Lee, Lee, Lee \& Yoo. The essential role of FKBP38 in regulating phosphatase of regenerating liver 3 (PRL-3) protein stability. Biochem. Biophys. Res. Commun. 406, 305-309 (2011).

143. Xing, Lu, Guo, Shen, Zhang, He, Gan, Li, Wang, Zhao, Wu \& Li. UbiquitinSpecific Protease 4-Mediated Deubiquitination and Stabilization of PRL-3 Is Required for Potentiating Colorectal Oncogenesis. Cancer Res. 76, 83-95 (2016).

144. Lin, Lee, Wang, Li, Hsien, Cheng, Chang, Huang, Yu \& Chen. Expression of phosphatase of regenerating liver family genes during embryogenesis: an evolutionary developmental analysis among Drosophila, amphioxus, and zebrafish. BMC Dev. Biol. 13, 18 (2013).

145. Dumaual, Sandusky, Crowell \& Randall. Cellular Localization of PRL-1 and PRL2 Gene Expression in Normal Adult Human Tissues. J. Histochem. Cytochem. 54, 1401-1412 (2006).

146. Moores, Schaber, Mosser, Rands, O’Hara, Garsky, Marshall, Pompliano \& Gibbs. Sequence dependence of protein isoprenylation. J. Biol. Chem. 266, 14603-10 (1991).

147. Zhang \& Casey. Protein Prenylation: Molecular Mechanisms and Functional Consequences. Annu. Rev. Biochem. 65, 241-269 (1996).

148. Song, Qian, Li, Sheng, Cao \& Xu. Phosphatase of regenerating liver-3 localizes to cyto-membrane and is required for $\mathrm{B} 16 \mathrm{~F} 1$ melanoma cell metastasis in vitro and in vivo. PLoS One 4, e4450 (2009).

149. Hancock, Paterson \& Marshall. A polybasic domain or palmitoylation is required in addition to the CAAX motif to localize p21ras to the plasma membrane. Cell 63, 133-9 (1990).

150. McParland, Varsano, Li, Thornton, Baby, Aravind, Meyer, Pavic, Rios \& Köhn. 
The metastasis-promoting phosphatase PRL-3 shows activity toward phosphoinositides. Biochemistry 50, 7579-90 (2011).

151. Foy, Anézo, Saule \& Planque. PRL-3/PTP4A3 phosphatase regulates integrin $\beta 1$ in adhesion structures during migration of human ocular melanoma cells. Exp. Cell Res. 353, 88-99 (2017).

152. Tian, Qu, Meng, Liu, Wu \& Shou. Phosphatase of regenerating liver-3 directly interacts with integrin $\beta 1$ and regulates its phosphorylation at tyrosine 783. BMC Biochem. 13, 22 (2012).

153. Krndija, Münzberg, Maass, Hafner, Adler, Kestler, Seufferlein, Oswald \& von Wichert. The phosphatase of regenerating liver 3 (PRL-3) promotes cell migration through Arf-activity-dependent stimulation of integrin $\alpha 5$ recycling. J. Cell Sci. 125, 3883-92 (2012).

154. Liang, Liang, Wang, Sun, Udho \& Zhang. PRL3 Promotes Cell Invasion and Proliferation by Down-regulation of Csk Leading to Src Activation. J. Biol. Chem. 282, 5413-5419 (2007).

155. Peng, Jin, Wang, Guo, Meng \& Shou. Identification of integrin alpha1 as an interacting protein of protein tyrosine phosphatase PRL-3. Biochem. Biophys. Res. Commun. 342, 179-83 (2006).

156. Balla. Phosphoinositides: Tiny Lipids With Giant Impact on Cell Regulation. Physiol. Rev. 93, 1019-1137 (2013).

157. Hammond, Fischer, Anderson, Holdich, Koteci, Balla \& Irvine. PI4P and PI(4,5)P2 Are Essential But Independent Lipid Determinants of Membrane Identity. Science (80-. ). 337, 727-730 (2012).

158. Godi, Campli, Konstantakopoulos, Tullio, Alessi, Kular, Daniele, Marra, Lucocq \& Matteis. FAPPs control Golgi-to-cell-surface membrane traffic by binding to ARF and PtdIns(4)P. Nat. Cell Biol. 6, 393-404 (2004).

159. Tsukita \& Yonemura. Cortical actin organization: lessons from ERM (ezrin/radixin/moesin) proteins. J. Biol. Chem. 274, 34507-10 (1999).

160. Parnell, Koschinski, Zaccolo, Cameron, Baillie, Baillie, Porter, McElroy \& Yarwood. Phosphorylation of ezrin on Thr567 is required for the synergistic activation of cell spreading by EPAC1 and protein kinase A in HEK293T cells. Biochim. Biophys. Acta - Mol. Cell Res. 1853, 1749-1758 (2015).

161. Antelmi, Cardone, Greco, Rubino, Di Sole, Martino, Casavola, Carcangiu, Moro \& Reshkin. B1 Integrin Binding Phosphorylates Ezrin at T567 to Activate a Lipid Raft Signalsome Driving Invadopodia Activity and Invasion. PLoS One 8, e75113 (2013).

162. Walls, Iliuk, Bai, Wang, Tao \& Zhang. Phosphatase of regenerating liver 3 (PRL3) provokes a tyrosine phosphoproteome to drive prometastatic signal transduction. Mol. Cell. Proteomics 12, 3759-77 (2013).

163. Liu, Al-aidaroos, Wang, Guo, Li, Zhang \& Zeng. PRL-3 suppresses c-Fos and integrin $\alpha 2$ expression in ovarian cancer cells. BMC Cancer 13, 80 (2013).

164. Moser, Legate, Zent \& Fassler. The Tail of Integrins, Talin, and Kindlins. Science (80-. ). 324, 895-899 (2009).

165. Tapley, Horwitz, Buck, Duggan \& Rohrschneider. Integrins isolated from Rous sarcoma virus-transformed chicken embryo fibroblasts. Oncogene 4, 325-33 (1989). 
166. Sakai, Jove, Fassler \& Mosher. Role of the cytoplasmic tyrosines of $1 \mathrm{~A}$ integrins in transformation by v-src. Proc. Natl. Acad. Sci. 98, 3808-3813 (2001).

167. Bledzka, Bialkowska, Nie, Qin, Byzova, Wu, Plow \& Ma. Tyrosine Phosphorylation of Integrin 3 Regulates Kindlin-2 Binding and Integrin Activation. J. Biol. Chem. 285, 30370-30374 (2010).

168. Meves, Geiger, Zanivan, DiGiovanni, Mann \& Fässler. Beta1 integrin cytoplasmic tyrosines promote skin tumorigenesis independent of their phosphorylation. Proc. Natl. Acad. Sci. U. S. A. 108, 15213-8 (2011).

169. Nilsson, Kaniowska, Brakebusch, Fässler \& Johansson. Threonine 788 in integrin subunit $\beta 1$ regulates integrin activation. Exp. Cell Res. 312, 844-853 (2006).

170. Wennerberg, Fassler, Warmegard \& Johansson. Mutational analysis of the potential phosphorylation sites in the cytoplasmic domain of integrin beta1A. Requirement for threonines 788-789 in receptor activation. J. Cell Sci. 111, (1998).

171. Wennerberg, Armulik, Sakai, Karlsson, Fässler, Schaefer, Mosher \& Johansson. The cytoplasmic tyrosines of integrin subunit beta1 are involved in focal adhesion kinase activation. Mol. Cell. Biol. 20, 5758-65 (2000).

172. Suzuki \& Takahashi. Reduced cell adhesion during mitosis by threonine phosphorylation of ?1 integrin. J. Cell. Physiol. 197, 297-305 (2003).

173. Nakajima, Suzuki \& Takahashi. Mitotic dissociation of IQGAP1 from Rac-bound $\beta 1$-integrin is mediated by protein phosphatase 2A. Biochem. Biophys. Res. Commun. 326, 249-253 (2004).

174. Stroeken, van Rijthoven, de Boer, Geerts \& Roos. Cytoplasmic domain mutants of $\beta 1$ integrin, expressed in $\beta 1$-knockout lymphoma cells, have distinct effects on adhesion, invasion and metastasis. Oncogene 19, 1232-1238 (2000).

175. Kuroda, Fukata, Kobayashi, Nakafuku, Nomura, Iwamatsu \& Kaibuchi. Identification of IQGAP as a putative target for the small GTPases, Cdc42 and Rac1. J. Biol. Chem. 271, 23363-7 (1996).

176. Didsbury, Weber, Bokoch, Evans \& Snyderman. rac, a novel ras-related family of proteins that are botulinum toxin substrates. J. Biol. Chem. 264, 16378-82 (1989).

177. Bishop \& Hall. Rho GTPases and their effector proteins. Biochem. J. 348 Pt 2, 241-55 (2000).

178. Michaelson, Abidi, Guardavaccaro, Zhou, Ahearn, Pagano \& Philips. Rac1 accumulates in the nucleus during the $\mathrm{G} 2$ phase of the cell cycle and promotes cell division. J. Cell Biol. 181, 485-96 (2008).

179. Lawson \& Burridge. The on-off relationship of Rho and Rac during integrinmediated adhesion and cell migration. Small GTPases 5, (2014).

180. Wertheimer, Gutierrez-Uzquiza, Rosemblit, Lopez-Haber, Sosa \& Kazanietz. Rac signaling in breast cancer: A tale of GEFs and GAPs. Cell. Signal. 24, 353-362 (2012).

181. Kovac, Teo, Mäkelä \& Vallenius. Assembly of non-contractile dorsal stress fibers requires $\alpha$-actinin-1 and Rac1 in migrating and spreading cells. J. Cell Sci. 126, 263-73 (2013).

182. Davis, Ha, Holman, Halaban, Schlessinger \& Boggon. RAC1P29S is a spontaneously activating cancer-associated GTPase. Proc. Natl. Acad. Sci. U. S. A. 110, 912-7 (2013). 
183. Grizot, Faure, Fieschi, Vignais, Dagher \& Pebay-Peyroula. Crystal Structure of the Rac1 - RhoGDI Complex Involved in NADPH Oxidase Activation. Biochem. 2001, 40, 10007-10013 (2001).

184. Pai, Kim \& Williams. Rac GTPases in human diseases. Dis. Markers 29, 177-87 (2010).

185. Navarro-Lérida, Sánchez-Perales, Calvo, Rentero, Zheng, Enrich \& Del Pozo. A palmitoylation switch mechanism regulates Rac1 function and membrane organization. EMBO J. 31, 534-51 (2012).

186. Shao, Yue, Sun, You, Wang \& Zhang. Caveolin-1 regulates Rac1 activation and rat pulmonary microvascular endothelial hyperpermeability induced by TNF- $\alpha$. PLoS One 8, e55213 (2013).

187. Gungor, Gombos, Crul, Ayaydin, Szabó, Török, Mátés, Vígh \& Horváth. Rac1 Participates in Thermally Induced Alterations of the Cytoskeleton, Cell Morphology and Lipid Rafts, and Regulates the Expression of Heat Shock Proteins in B16F10 Melanoma Cells. PLoS One 9, e89136 (2014).

188. Michaely, Mineo, Ying \& Anderson. Polarized distribution of endogenous Rac1 and RhoA at the cell surface. J. Biol. Chem. 274, 21430-6 (1999).

189. Gaus, Le Lay, Balasubramanian \& Schwartz. Integrin-mediated adhesion regulates membrane order. J. Cell Biol. 174, (2006).

190. Balasubramanian, Meier, Scott, Norambuena, White \& Schwartz. RalA-Exocyst Complex Regulates Integrin-Dependent Membrane Raft Exocytosis and Growth Signaling. Curr. Biol. 20, 75-79 (2010).

191. Page, Li, Hodge, Liu, Vanden Hoek, Becker, Pestell, Rosner \& Hershenson. Characterization of a Rac1 signaling pathway to cyclin $\mathrm{D}(1)$ expression in airway smooth muscle cells. J. Biol. Chem. 274, 22065-71 (1999).

192. Coleman, Marshall \& Olson. RAS and RHO GTPases in G1-phase cell-cycle regulation. Nat. Rev. Mol. Cell Biol. 5, 355-366 (2004).

193. Mettouchi, Klein, Guo, Lopez-Lago, Lemichez, Westwick \& Giancotti. IntegrinSpecific Activation of Rac Controls Progression through the G1 Phase of the Cell Cycle. Mol. Cell 8, 115-127 (2001).

194. Yoshida, Zhang, Rivera Rosado, Chen, Khan, Moon \& Zhang. Blockade of Rac1 activity induces G1 cell cycle arrest or apoptosis in breast cancer cells through downregulation of cyclin D1, survivin, and X-linked inhibitor of apoptosis protein. Mol. Cancer Ther. 9, 1657-68 (2010).

195. Liu, Zhang, Shi, Zhang, Yuan, Chen, Liu, Zhang \& Wang. Inhibition of Rac1 activity induces G1/S phase arrest through the GSK3/cyclin D1 pathway in human cancer cells. Oncol. Rep. 32, 1395-400 (2014).

196. Joyce, Bouzahzah, Fu, Albanese, D’Amico, Steer, Klein, Lee, Segall, Westwick, Der \& Pestell. Integration of Rac-dependent regulation of cyclin D1 transcription through a nuclear factor-kappaB-dependent pathway. J. Biol. Chem. 274, 25245-9 (1999).

197. Diehl, Cheng, Roussel \& Sherr. Glycogen synthase kinase-3beta regulates cyclin D1 proteolysis and subcellular localization. Genes Dev. 12, 3499-511 (1998).

198. Muise-Helmericks, Grimes, Bellacosa, Malstrom, Tsichlis \& Rosen. Cyclin D expression is controlled post-transcriptionally via a phosphatidylinositol 3kinase/Akt-dependent pathway. J. Biol. Chem. 273, 29864-72 (1998). 
199. Higuchi, Onishi, Kikuchi \& Gotoh. Scaffolding function of PAK in the PDK1-Akt pathway. Nat. Cell Biol. 10, 1356-1364 (2008).

200. Somanath, Vijai, Kichina, Byzova \& Kandel. The role of PAK-1 in activation of MAP kinase cascade and oncogenic transformation by Akt. Oncogene 28, 2365-9 (2009).

201. Castro-Castro, Ojeda, Barreira, Sauzeau, Navarro-Lérida, Muriel, Couceiro, Pimentel-Muíños, Del Pozo \& Bustelo. Coronin 1A promotes a cytoskeletal-based feedback loop that facilitates Rac1 translocation and activation. EMBO J. 30, 3913-27 (2011).

202. Symons. Rac1 activation comes full circle. EMBO J. 30, 3875-7 (2011).

203. Kilkenny \& Altman. Improving bioscience research reporting: ARRIVE-ing at a solution. Lab. Anim. 44, 377-378 (2010).

204. Torok, Vegvari, Rezeli, Fehniger, Tovari, Paku, Laszlo, Hegedus, Rozsas, Dome \& Marko-Varga. Localization of sunitinib, its metabolites and its target receptors in tumour-bearing mice: a MALDI-MS imaging study. Br. J. Pharmacol. 172, $1148-1163$ (2015).

205. Rózsás, Berta, Rojkó, Horváth, Keszthelyi, Kenessey, László, Berger, Grusch, Hoda, Török, Klepetko, Rényi-Vámos, Hegedűs, Döme \& Tóvári. Erythropoietin Receptor Expression Is a Potential Prognostic Factor in Human Lung Adenocarcinoma. PLoS One 8, e77459 (2013).

206. Maeda, Tanaka \& Akamatsu. Phospholipid methylation in MOPC-31C cell membranes with modified phospholipid composition. Biochem. Biophys. Res. Commun. 96, 876-881 (1980).

207. Song, Li Shengwen, Okamoto, Quilliam, Sargiacomo \& Lisanti. Co-purification and direct interaction of Ras with caveolin, an integral membrane protein of caveolae microdomains. Detergent-free purification of caveolae microdomains. $J$. Biol. Chem. 271, 9690-7 (1996).

208. Sankaran, Shi, Ho, Stelzer \& Wohland. ImFCS: A software for Imaging FCS data analysis and visualization. Opt. Express 18, 25468 (2010).

209. Ng, Bag \& Wohland. Characterization of Lipid and Cell Membrane Organization by the Fluorescence Correlation Spectroscopy Diffusion Law. Chim. Int. J. Chem. 69, 112-119 (2015).

210. Balogh, Maulucci, Gombos, Horváth, Török, Péter, Fodor, Páli, Benko, Parasassi, De Spirito, Harwood \& Vígh. Heat stress causes spatially-distinct membrane remodelling in K562 leukemia cells. PLoS One 6, e21182 (2011).

211. Marks, Bittman \& Pagano. Use of Bodipy-labeled sphingolipid and cholesterol analogs to examine membrane microdomains in cells. Histochem. Cell Biol. 130, 819-32 (2008).

212. Chen, Yang \& Jacobson. Transient confinement zones: a type of lipid raft? Lipids 39, 1115-9 (2004).

213. Qian, Li, Sheng, Zhang, Song, Dong, Cao, Hua \& Xu. PRL-3 siRNA inhibits the metastasis of B16-BL6 mouse melanoma cells in vitro and in vivo. Mol. Med. 13, $151-9$

214. Wu, Zeng, Zhang, Zhao, Sha, Ge, Zhang, Gao \& Xu. Phosphatase of regenerating liver-3 promotes motility and metastasis of mouse melanoma cells. Am. J. Pathol. 164, 2039-54 (2004). 
215. Poste, Doll, Hart \& Fidler. In vitro selection of murine B16 melanoma variants with enhanced tissue-invasive properties. Cancer Res. 40, 1636-44 (1980).

216. Guo, Li, Tang, Tan, Hong, Al-Aidaroos, Varghese, Huang \& Zeng. Targeting intracellular oncoproteins with antibody therapy or vaccination. Sci. Transl. Med. 3, 99ra85 (2011).

217. Ganapathi, Schmidt, Grabowski, Melia \& Ratliff. Modulation in vitro and in vivo of cytotoxicity but not cellular levels of doxorubicin by the calmodulin inhibitor trifluoperazine is dependent on the level of resistance. Br. J. Cancer 58, 335-40 (1988).

218. Alemón-Medina, Bravo-Gómez, Gracia-Mora \& Ruiz-Azuara. Comparison between the antiproliferative effect and intracellular glutathione depletion induced by Casiopeína IIgly and cisplatin in murine melanoma B16 cells. Toxicol. Vitr. 25, 868-873 (2011).

219. Garnett, Edelman, Heidorn, Greenman, Dastur, Lau, Greninger, Thompson, Luo, Soares, Liu, Iorio, Surdez, Chen, Milano, Bignell, Tam, Davies, Benes, et al. Systematic identification of genomic markers of drug sensitivity in cancer cells. Nature 483, 570-575 (2012).

220. Rouleau, Roy, St Martin, Dufault, Boutin, Liu, Zhang, Puorro-Radzwill, Rulli, Reczek, Bagley, Byrne, Weber, Roberts, Klinger, Brondyk, Nacht, Madden, Burrier, Shankara \& Teicher. Protein tyrosine phosphatase PRL-3 in malignant cells and endothelial cells: expression and function. Mol. Cancer Ther. 5, 219-29 (2006).

221. Thorgeirsson, Turpeenniemi-Hujanen, Williams, Westin, Heilman, Talmadge \& Liotta. NIH/3T3 cells transfected with human tumor DNA containing activated ras oncogenes express the metastatic phenotype in nude mice. Mol. Cell. Biol. 5, 25962 (1985).

222. Suda, Aizawa, Furuta, Yagi, Ikawa, Saitoh, Yamada, Toyoshima \& Yamamoto. Induction of a variety of tumors by c-erbB2 and clonal nature of lymphomas even with the mutated gene (Val659----Glu659). EMBO J. 9, 181-90 (1990).

223. Meng, Cheng, Krohkin, Mould, Humphries, Ens, Standing \& Wilkins. Evidence for the presence of a low-mass beta1 integrin on the cell surface. J. Cell Sci. 118, 4009-16 (2005).

224. Gao, Dickerson, Guo, Zheng \& Zheng. Rational design and characterization of a Rac GTPase-specific small molecule inhibitor. Proc. Natl. Acad. Sci. U. S. A. 101, 7618-23 (2004).

225. Lingwood, Ries, Schwille \& Simons. Plasma membranes are poised for activation of raft phase coalescence at physiological temperature. Proc. Natl. Acad. Sci. 105, 10005-10010 (2008).

226. Hogue, Grover, Soheilian, Nagashima \& Ono. Gag induces the coalescence of clustered lipid rafts and tetraspanin-enriched microdomains at HIV-1 assembly sites on the plasma membrane. J. Virol. 85, 9749-66 (2011).

227. Hofman, Ruonala, Bader, van den Heuvel, Voortman, Roovers, Verkleij, Gerritsen $\&$ van Bergen en Henegouwen. EGF induces coalescence of different lipid rafts. $J$. Cell Sci. 121, 2519-2528 (2008).

228. Ayuyan \& Cohen. Raft composition at physiological temperature and $\mathrm{pH}$ in the absence of detergents. Biophys. J. 94, 2654-66 (2008). 
229. Bromberg, Goloubinoff, Saidi \& Weiss. The Membrane-Associated Transient Receptor Potential Vanilloid Channel Is the Central Heat Shock Receptor Controlling the Cellular Heat Shock Response in Epithelial Cells. PLoS One 8, e57149 (2013).

230. Balogh, Péter, Liebisch, Horváth, Török, Nagy, Maslyanko, Benko, Schmitz, Harwood \& Vígh. Lipidomics reveals membrane lipid remodelling and release of potential lipid mediators during early stress responses in a murine melanoma cell line. Biochim. Biophys. Acta 1801, 1036-47 (2010).

231. Han, Oh, Woo, Kim, Kim, Kim \& Kang. Implication of a small GTPase Rac1 in the activation of c-Jun N-terminal kinase and heat shock factor in response to heat shock. J. Biol. Chem. 276, 1889-95 (2001).

232. Nagy, Balogi, Gombos, Akerfelt, Björkbom, Balogh, Török, Maslyanko, FiszerKierzkowska, Lisowska, Slotte, Sistonen, Horváth \& Vígh. Hyperfluidizationcoupled membrane microdomain reorganization is linked to activation of the heat shock response in a murine melanoma cell line. Proc Natl Acad Sci U S A 104, 7945-7950 (2007).

233. Tabouillot, Muddana \& Butler. Endothelial Cell Membrane Sensitivity to Shear Stress is Lipid Domain Dependent. Cell. Mol. Bioeng. 4, 169-181 (2011).

234. Anderson, Tao, Betten \& Hahn. Heat shock protein levels are not elevated in heatresistant B16 melanoma cells. Radiat. Res. 105, 240-6 (1986).

235. Shigapova, Török, Balogh, Goloubinoff, Vígh \& Horváth. Membrane fluidization triggers membrane remodeling which affects the thermotolerance in Escherichia coli. Biochem Biophys Res Commun 328, 1216-1223 (2005).

236. Swan \& Watson. Stress tolerance in a yeast lipid mutant: membrane lipids influence tolerance to heat and ethanol independently of heat shock proteins and trehalose. Can. J. Microbiol. 45, 472-9 (1999).

237. Qu, Liu, Guo, Shi, Zhou, Li, Yang, Tong, Wang, MD, Li, BS, Yang, PhD, Tong, $\mathrm{MD}, \mathrm{PhD}$, Wang \& PhD. Independent oncogenic and therapeutic significance of phosphatase PRL-3 in FLT3-ITD-negative acute myeloid leukemia. Cancer 120, 2130-41 (2014).

238. Melnikova, Bolshakov, Walker \& Ananthaswamy. Genomic alterations in spontaneous and carcinogen-induced murine melanoma cell lines. Oncogene 23, $2347-2356$ (2004).

239. Sebag, Stewart, Shi, Zhu, Chng, Keats, Tiedemann, Fonseca, Chesi \& Bergsagel. PRL3 Is a Mediator of IL6/STAT3 Signaling and Defines a Population of Multiple Myeloma Patients Distinct from Those That Activate NFkB. Blood 110, (2007).

240. Moreno-Layseca \& Streuli. Signalling pathways linking integrins with cell cycle progression. Matrix Biol. 34, 144-153 (2014).

241. Smith, Marra \& Marshall. uPAR promotes formation of the p130Cas-Crk complex to activate Rac through DOCK180. J. Cell Biol. 182, 777-790 (2008).

242. Gaus, Chklovskaia, Fazekas de St. Groth, Jessup \& Harder. Condensation of the plasma membrane at the site of T lymphocyte activation. J. Cell Biol. 171, 121131 (2005).

243. Del Pozo \& Schwartz. Rac, membrane heterogeneity, caveolin and regulation of growth by integrins. Trends Cell Biol. 17, 246-50 (2007).

244. Koleske, Baltimore \& Lisanti. Reduction of caveolin and caveolae in 
oncogenically transformed cells. Proc. Natl. Acad. Sci. U. S. A. 92, 1381-5 (1995).

245. Bender, Reymond, Bron \& Quest. Caveolin-1 levels are down-regulated in human colon tumors, and ectopic expression of caveolin-1 in colon carcinoma cell lines reduces cell tumorigenicity. Cancer Res. 60, 5870-8 (2000).

246. Tryfonopoulos, Walsh, Collins, Flanagan, Quinn, Corkery, McDermott, Evoy, Pierce, O'Donovan, Crown \& Duffy. Src: a potential target for the treatment of triple-negative breast cancer. Ann. Oncol. 22, 2234-2240 (2011).

247. Gari, DeGala, Ray, Lucia \& Lambert. PRL-3 engages the focal adhesion pathway in triple-negative breast cancer cells to alter actin structure and substrate adhesion properties critical for cell migration and invasion. Cancer Lett. 380, 505-512 (2016).

248. Bełkot, Bubka \& Lityńska. Expression of Caveolin-1 in Human Cutaneous and Uveal Melanoma Cells. Folia Biol. (Praha). 64, 145-151 (2016). 


\section{Summary}

By being the outermost boundary of the cell, the plasma membrane is deeply involved in the sensing and transmission of signals from the environment towards the cellular interior. Lipid rafts are creating a great degree of membrane heterogeneity and by this they are allowing a higher level of compartmentalization to happen in the plasma membrane. This compartmentalization allows the lipid rafts to act like sorting platforms for signal transduction proteins. Many proteins assembled in lipid rafts are involved in a wide range of signaling processes ranging from stress sensing, cell survival and growth signaling. The thesis characterizes two important aspects of how the plasma membrane functions in times of stress and disease.

The first part of the thesis focuses on aspects of the phenomena of the membrane stress sensor model. My research revealed a significant role for the fluidization induced HSP25 expression in the maintenance of membrane homeostasis. I was able to describe the upregulation of HSP25 at the protein level by applying an artificial membrane fluidizer. My investigations also led to the discovery of a so far unknown feature of HSP25, which was materializing in its effect to act against forced membrane fluidization. Therefore I describe a new feature for HSP25 as a membrane buffering agent which helps to maintain the general structure of the membrane. Also by using such "semi-in vitro" system as the isolated membrane sheets I was able to further narrow down the possible interactions of HSP25 to membrane lipids and/or transmembrane proteins.

In the second part of the thesis I studied the PRL3 protein, a membrane associated oncogene. Hereby, I describe an intriguing upregulation of the PRL3 protein in cancer cells upon exposure to genotoxic chemotherapeutic agents. Moreover my experiments were able to successfully connect the presence of PRL3 with a distinct phenotype that could further tumor progression by affecting tumor growth. My observations also have led me to conclude that PRL3 resides primarily at the caveolae domains of the plasma membrane. Furthermore my experiments were able to propose a possible substrate for the protein and describe the molecular signaling background behind its action. 


\section{9 Összefoglaló}

A plazma membrán azon helyzetéből adódóan, hogy a sejt legkülsőbb határát képezi, központi szerepet játszik a környezetből érkező jelek érzékelésében és továbbításában. A lipid raft struktúrák nagymértékben felelősek a membrán heterogenitásának kialakításában és ezzel lehetővé teszik egy magasabb szintü membrán-szervezettség létrejöttét. A membrán heterogenitásának nagy szerepe van különbözö jelátviteli folyamatokban, mivel lehetővé teszi egyes jelátviteli molekulák platformokba való rendeződését. A lipid raft struktúrákban található fehérjék komoly szerepet játszanak több sejt esemény, mint pl. a stressz érzékelés, sejt növekedés és túlélés, jelátviteli folyamataiban. A doktori disszertáció keretében bemutatott munka elsősorban a lipid raftok által szabályozott jelátvitel két aspektusát vizsgálja.

A munka első része mélyebb betekintést ad a membrán struktúrát befolyásoló stressz következtében fellépő membrán adaptációs folyamatok mechanizmusába fókuszálva a stressz következtében fellépő stressz fehérje indukcióra. A membrán stresszel kapcsolatos kutatásaim rámutattak a HSP25 fehérje központi szerepére a membrán homeosztázis fenntartásában. Kísérleteimben sikerült a HSP25 fehérje kifejeződésének emelkedését kimutatnom mesterséges membrán fluidizáció hatására. Továbbá a kutatásaim fényt derítettek a HSP25 egy eddig ismeretlen tulajdonságára, ami a fehérje membrán fluidizációt kivédő hatásában nyilvánult meg. Ezen kísérletek által sikerült a HSP25-t egy membrán puffer kapacitást biztosító ágensként karakterizálnom, amely stressz hatás alatt segít a membrán struktúra fenntartásában. Ezen túl a HSP25 lehetséges membrán interakcióját sikeresen szűkítettem le a membránnal való közvetlen interakcióra.

A disszertáció második része a membrán tumoros folyamatokban kifejtett szerepére koncentrált egy PRL3 nevü membrán asszociált onkogén vizsgálata által. A kísérleteimben sikeresen írtam le a PRL3 fehérje kifejeződésének növekedését DNS károsító anti-tumor terapeutikumok hatására, tumor sejtekben. Ezen túl kutatásaim kapcsolatba hozták a terápia hatására megemelkedett PRL3 kifejeződését a tumor sejt megnövekedett növekedési képességével. A PRL3 hatásmechanizmusát vizsgáló kiserleteimben sikerült karakterizálnom a fehérje asszociációját a caveola típusú lipid raft doménekhez. Valamint ebből kiindulva sikeresen írtam le a PRL3 expresszió hatásával összefüggő jelátviteli változásokat. 


\section{Acknowledgements}

First of all, I would like to express my gratitude to my supervisor Prof. László Vígh for his guidance, encouragement and contributions throughout my studies. It was a great pleasure to discuss science with him. I would also like to thank for giving me the opportunity to work in such a friendly environment. I am sincerely grateful to Dr. Ibolya Horváth for her advices and constructive critics.

I feel obliged to be part of the Molecular Stress Biology team in the BRC. I appreciate the help, the contribution and the valuable friendships of Imre Gombos, Gábor Balogh, Mária Péter, Tim Crul, Zsolt, Török, Ana-Maria Pilbat, Ákos Hunya, Atilla Glatz, Péter Gudman, Ádám Tiszlavicz. Gabi Bogdánné, Aranka Gondáné. I would also like to thank our Erika Zuric for, and Éva Bartha for their help during my work.

It was a great experience to work with our collaborator Dr. Anna Kiss from the Semmelweis University. I am also thankful to Dr. József Tóvári for allowing me access to his lab and animal facility at the National Center of Oncology at Budapest, and also to Dr. Enikő Tátrai for her invaluable help in carrying out the experiments.

I would like to thank Dr. Lajos Mátés and Dr. Csaba Sőti for reviewing my thesis.

Besides the scientific part, it is essential to thank my friends for their friendship and encouragement. At last but not least, I am very thankful for the endless support of my family. It wouldn't be possible for me to complete my work without these.

This work was supported by the Ministry for National Economy (GINOP-2.3.2-15-201600001, GINOP-2.3.2-15-2016-00040 and GINOP-2.3.2-15-2016-00060). 


\section{Appendices}

\subsection{Publication List}

MTMT number: 10038350

Publications that are related to the $\mathrm{PhD}$ thesis:

Csoboz B, Balogh GE, Kusz E, Gombos I, Peter M, Crul T, Gungor B, Haracska L, Bogdanovics G, Torok Z, Horvath I, Vigh L.

Membrane fluidity matters: hyperthermia from the aspects of lipids and membranes.

Int J Hyperthermia. 2013 Aug; 29(5):491-9. doi: 10.3109/02656736.2013.808765. Epub 2013 Jul 10.

I.F.: 2.645

Török Z, Crul T, Maresca B, Schütz GJ, Viana F, Dindia L, Piotto S, Brameshuber M, Balogh G, Péter M, Porta A, Trapani A, Gombos I, Glatz A, Gungor B, Peksel B, Vigh L Jr, Csoboz B, Horváth I, Vijayan MM, Hooper PL, Harwood JL, Vigh L.

Plasma membranes as heat stress sensors: from lipid-controlled molecular switches to therapeutic applications.

Biochim Biophys Acta. 2014 Jun; 1838(6):1594-618. doi: 10.1016/j.bbamem.2013.12.015. Epub 2013 Dec 27.

I.F.: 3,498

Presentations in meetings and conferences:

Csoboz B, Gombos I, Tovari J, Kiss AL, Peter M, Balogh G, Horvath I, Vigh L Structural changes of the plasma membrane upon tumorigenesis EMBO workshop: Experimental and Theoretical approaches to cell mechanics, 2017, poster presentation

Csoboz B, Gombos I, Tovari J, Peter J, Balogh G, Horvath I, Vigh L

Changes in the membrane structure is associated with enchanced cancer cell growth upon oncogenic stimulation

EMBO conference: Cellular signaling and cancer therapy, 2016, poster presentation 
Csoboz B, Gombos I, Tovari J, Peter M, Balogh G, Huang B; Wang N; Horvath I, Vigh L Membrane associated stress response within the context of cancer progression

EMBO-FEBS lecture course: Biomembranes: Molecular Architecture, Dynamics and Function, 2015, poster presentation

Csoboz B, Balogh G, Gombos I, Peter M, Horvath I, Vigh L

Rearrangement of cholesterol rich membrane domains result in an acquired thermotolerant phenotype of B16 melanoma cells

FEBS workshop: Lipids as molecular switches, 2014, oral presentation 


\subsection{Supplementary Figures}

\section{$\begin{array}{ccccc} & \text { B16F10 } & \text { B16F10/HSP25 } & \text { B16F10 } \\ 42^{\circ} \mathrm{C} & 37^{\circ} \mathrm{C} & \text { Dox. } & \text { w/o Dox. Dox. } & \text { w/o Dox. }\end{array}$}

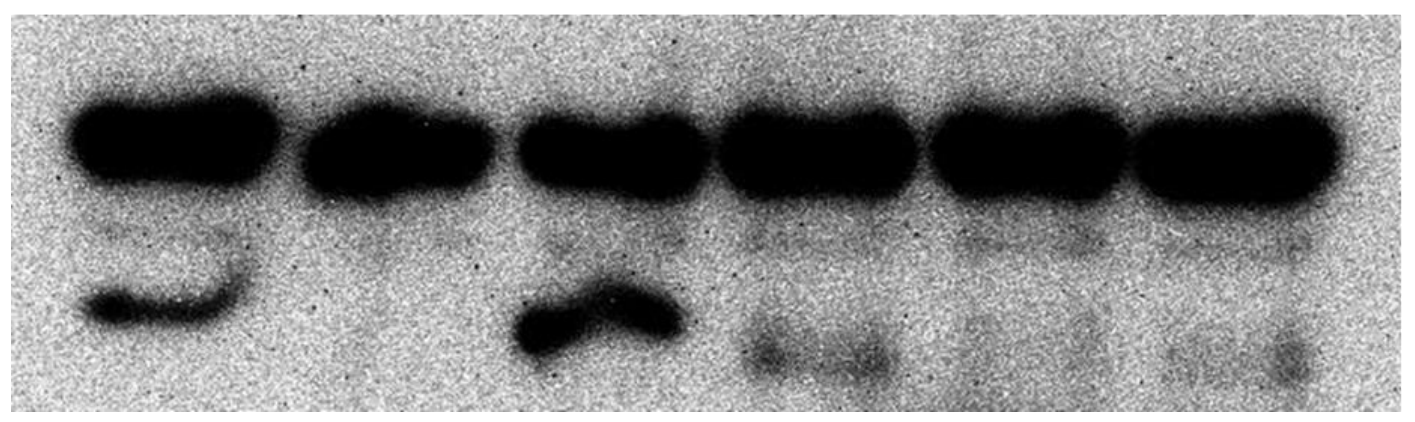

anti-GAPDH

Supplementary Figure 1.

Comparison between artificial and heat shock induced HSP25 expression induction. B16F10 cells subjected to heat treatment for 1 hour at $42^{\circ} \mathrm{C}\left(42^{\circ} \mathrm{C}\right)$ left untreated $\left(37^{\circ} \mathrm{C}, \mathrm{w} / \mathrm{o}\right.$ Dox.) or treated with $2 \mu \mathrm{g} / \mathrm{ml}$ doxycycline for 24 hour. B16F10/HSP25 cells were treated with 2 $\mu \mathrm{g} / \mathrm{ml}$ doxycycline for 24 hours or left untreated. Protein expression changes were analyzed by western blotting after 24 following each treatment.

1. 2. 3. 4. 5. 6. 7. $\quad$ 8. $9 . \quad 10 . \quad 11.12 .13$.

anti-Integrin beta 1

\section{Supplementary Figure 2.}

The distribution of integrin beta 1 between different plasma membrane fractions.

The plasma membrane of B16F0 cells were fractionated by ultracentrifugation as detailed above. The distribution of integrin beta 1 was determined by western blotting. 